\title{
Reinforcing interpenetrating network hydrogels with 3D printed polymer networks to engineer cartilage mimetic composites
}

\author{
Rossana Schipani $^{1,3}$, Stefan Scheurer ${ }^{1,2}$, Romain Florentin ${ }^{1,3}$, Susan E Critchley ${ }^{1,3}$ and Daniel J Kelly ${ }^{1,3,4,5}$ \\ 1 Trinity Centre for Biomedical Engineering, Trinity Biomedical Sciences Institute, Trinity College Dublin, Dublin, Ireland \\ 2 Orthopaedics and Sports Medicine, Trinity College Dublin, Dublin, Ireland \\ 3 Department of Mechanical and Manufacturing Engineering, School of Engineering, Trinity College Dublin, Ireland \\ 4 Department of Anatomy, Royal College of Surgeons in Ireland, Dublin, Ireland \\ 5 Advanced Materials and Bioengineering Research Centre (AMBER), Royal College of Surgeons in Ireland and Trinity College Dublin, \\ Dublin, Ireland \\ E-mail: kellyd9@tcd.ie
}

Received xxxxxx

Accepted for publication $\mathrm{xxxxxx}$

Published xxxxxx

\begin{abstract}
Engineering constructs that mimic the complex structure, composition and biomechanics of the articular cartilage represents a promising route to joint regeneration. Such tissue engineering strategies require the development of biomaterials that mimic the mechanical properties of articular cartilage whilst simultaneously providing an environment supportive of chondrogenesis. Here three-dimensional (3D) bioprinting is used to develop polycaprolactone (PCL) fibre networks to mechanically reinforce interpenetrating network (IPN) hydrogels consisting of alginate and gelatin methacryloyl (GelMA). Inspired by the significant tension-compression nonlinearity of the collagen network in articular cartilage, we printed reinforcing PCL networks with different ratios of tensile to compressive modulus. Synergistic increases in compressive modulus were observed when IPN hydrogels were reinforced with PCL networks that were relatively soft in compression and stiff in tension. The resulting composites possessed equilibrium and dynamic mechanical properties that matched or approached that of native articular cartilage. Finite Element (FE) modelling revealed that the reinforcement of IPN hydrogels with specific PCL networks limited radial expansion and increased the hydrostatic pressure generated within the IPN upon the application of compressive loading. Next, multiple-tool biofabrication techniques were used to 3D bioprint PCL reinforced IPN hydrogels laden with a co-culture of bone marrow-derived stromal cells (BMSCs) and chondrocytes (CCs). The bioprinted biomimetic composites were found to support robust chondrogenesis, with encapsulated cells producing hyaline-like cartilage that stained strongly for sGAG and type II collagen deposition, and negatively for type X collagen and calcium deposition. Taken together, these results demonstrate how 3D bioprinting can be used to engineer constructs that are both pro-chondrogenic and biomimetic of the mechanical properties of articular cartilage.
\end{abstract}

Keywords: articular cartilage, tissue engineering, interpenetrating network hydrogels, polycaprolactone, 3D bioprinting, finite element modelling, biomimetic scaffolds

\section{Introduction}

Articular cartilage is a thin layer of connective tissue that lines the ends of bones within synovial joints. Under normal physiological conditions, cartilage provides a smooth, lubricated surface for articulation that facilitates load support and distribution across the joint. Biomechanically it functions as a multiphasic, fibre-reinforced material that has anisotropic, nonlinear and viscoelastic properties [1-3]. The complex load bearing properties of articular cartilage strongly depend on the anisotropic nature of the tissue, consisting primarily of collagen and proteoglycans, and the associated tensioncompression nonlinearity $[1,2,4,5]$. The negatively charged 
proteoglycans within articular cartilage generate an osmotic swelling pressure, which is balanced by a pre-stress that is developed in the collagen network [3,6]. The compressive properties of cartilage typically increase as the proteoglycans content increases, and the loss of proteoglycans has been shown to make the tissue more prone to microdamage during mechanical loading [1, 7-10]. The architecture and organization of the collagen fibres determine the intrinsic tensile stiffness and strength of cartilage [10-12]. The tensile modulus of articular cartilage is significantly higher than the compressive modulus $[13,14]$. When cartilage is tested in unconfined compression, this tension-compression nonlinearity enhances fluid pressurization and elevates the dynamic modulus of the tissue $[15,16]$, as the collagen network restricts lateral expansion of the tissue during compressive loading. The relative importance of the different matrix components on the overall mechanical properties of articular cartilage has been studied by depleting cartilage samples of collagen and/or proteoglycans [2, 9, 10, 17]. For example, the compressive modulus of the proteoglycandepleted cartilage matrix may be as low as $3 \mathrm{kPa}$, representing less than $2 \%$ of the normal tissue modulus [18]. Therefore, the collagen in articular cartilage can be considered as a fibrillar matrix that primarily sustains tension. Biomaterial strategies that aim to mimic articular cartilage should consider such complexities in their design.

As articular cartilage has poor regenerative capacity, damage to this tissue impairs its mechanical function and can lead to an abnormal loading within the joint. If left untreated, the damage can progress to osteoarthritis of the joint, causing significant pain and disability. This motivates the need for new regenerative approaches capable of restoring the normal biomechanics of the joint.

A number of tissue engineered strategies to engineer cartilage replacements have been developed [19-27]. A typical approach is to design biomaterial environments capable of supporting chondrogenesis; this commonly involves combining cells and/or bioactive molecules with hydrogels as a scaffolding material due to their high water content and attractive transport properties [28-31]. Other than presenting pro-chondrogenic biological cues to the encapsulated cells, such biomaterial scaffolds must also provide a suitable mechanical environment capable of instructing cell fate as well as withstanding the challenging physiological loading conditions experienced in vivo upon implantation into the body [27]. Commonly used single network hydrogels (e.g. alginate, GelMA, agarose, polyethylene glycol, etc.) have been extensively studied and have been shown to provide an environment that is conducive to chondrogenesis. However, they are typically mechanically weak and unable to withstand the challenging environment of the joint. Recently, more complex hydrogel systems such as IPN hydrogels, characterized by the combination of multiple polymer networks have been investigated. Within such IPN hydrogels individual polymer networks are physically entangled with each other and crosslinked only with themselves using specific chemistries [32-35]. In general, such IPN hydrogels have displayed synergistic increases in mechanical properties, including increased toughness and elasticity when compared to the single hydrogel components. However, they do not mimic many of the features of load bearing tissues like articular cartilage, such as anisotropy and tensioncompression nonlinearity. Furthermore, engineering IPN hydrogels with bulk mechanical properties approaching that of articular cartilage typically requires very high hydrogel polymer concentrations, resulting in biomaterial environments that are not compatible with robust extracellular matrix (ECM) deposition by encapsulated cells [36-38].

To address these concerns, hydrogels can be reinforced with networks of fibres to produce composites with improved mechanical properties [2, 31, 39-41]. However, these techniques typically involve the use of complex biofabrication strategies (e.g. weaving, melt electrowriting) that are challenging to scale and personalize to specific patient anatomies. In contrast, Fused Deposition Modelling (FDM) can be used to 3D print fibre networks that are relatively simple to scale, with internal and external architectures that are easily defined based on medical imaging $[42,43]$. This is achieved using PCL, a synthetic thermoplastic polymer which has been extensively used in the field of 3D bioprinting [44]. Advantages include ease of shaping and manufacture due to its low meting temperature $\left(\sim 60^{\circ} \mathrm{C}\right)$, biocompatibility, elasticity and mechanical properties [45]. The mechanical properties of PCL scaffolds can also be tuned by modifying the molecular weight and the scaffold architecture [46]. Other synthetic polymers such as poly(lactic acid) (PLA) and poly(lactic-co-glycolic acid) (PLGA) are also biocompatible with FDM, with PLGA also allowing for more tailorable degradation rates [47-49]. A concern with the use of PLGA is the release of acidic products as the polymer degrades, which can induce inflammation [50, 51]. Another major disadvantage is the higher melting temperature of PLA and PLGA compared to PCL, which is not ideal when co-printing with live cells.

In this study, alginate - GelMA IPNs were reinforced with PCL networks printed using FDM to develop biomechanically competent composite constructs for cartilage tissue engineering. [30, 52]. Alginate is a widely used biomaterial in tissue engineering because of its excellent biocompatibility, relatively high control over stiffness and capability to form highly porous structures which facilitate the diffusion of large molecules in and out of the hydrogel [53-55]. However, alginate is often limited under mechanical loading due to its brittleness and low elasticity [56-58]. GelMA is a gelatinbased material that has high biocompatibility, degrades in response to matrix metalloproteinases (MMP), possesses 
RGD sequences that favour cell adhesion and is highly elastic $[59,60]$. GelMA also possesses relatively poor mechanical strength and toughness [59, 61, 62], however IPNs of alginate and GelMA have previously been shown to have superior mechanical properties to their individual hydrogel components and to be supportive of cell growth and differentiation [59]. Here, alginate, GelMA and alginate GelMA IPN hydrogel scaffolds were first mechanically characterized to investigate the benefit of combining the two single hydrogel components for engineering cartilage-mimetic hydrogels. These hydrogel bioinks were then reinforced with 3D printed PCL networks with architectures and mechanical properties inspired by the collagen network of articular cartilage. The reinforcement mechanism resulting from the combination of PCL networks with IPN hydrogels was examined using Finite Element Analysis (FEA). Finally, the ability of 3D bioprinted cell-laden PCL reinforced IPN constructs to support chondrogenesis was assessed in vitro over 6 weeks of culture.

\section{Materials and methods}

\subsection{Materials preparation}

Alginate was prepared by dissolving Alginate (PRONOVA UP LVG) in DMEM to make up a final concentration of $3.5 \%$ w/v. Cylindrical constructs were obtained by pouring the alginate solution into $2 \mathrm{~mm}$ high- $6 \mathrm{~mm}$ diameter agarose$\mathrm{CaCl}_{2}$ moulds (DMEM, 3\% w/v agarose, $45 \mathrm{mM} \mathrm{CaCl} 2$ ) and allowed to crosslink for $20 \mathrm{~min}$ at room temperature. GelMA synthesis was previously described [63]. Briefly, GelMA was synthesized by reaction of porcine type A gelatin (SigmaAldrich, average molecular weight $40-50 \mathrm{kDa}$ ) with methacrylic anhydride (Sigma-Aldrich) at $50{ }^{\circ} \mathrm{C}$ for $4 \mathrm{~h}$. To achieve a high degree of functionalization, $10 \mathrm{~mL}$ of methacrylic anhydride was added to a $10 \%$ w/v gelatin solution in phosphate-buffered saline (PBS) under stirring for $1 \mathrm{~h}$. The functionalized polymer was dialyzed against distilled water for 7 days at $40{ }^{\circ} \mathrm{C}$ to remove unreacted methacrylic anhydride, freeze-dried and stored at $-20{ }^{\circ} \mathrm{C}$ protected from light until further use. GelMA constructs were formed by dissolving GelMA in DMEM (including $0.05 \% \mathrm{v} / \mathrm{v}$ Irgacure (Sigma Aldrich) as photoinitiator) obtaining final concentrations of 5, 10 and $15 \%$ w/v. The GelMA solution was poured into polydimethylsiloxane (PDMS) moulds (same dimensions as for the agarose- $\mathrm{CaCl}_{2}$ moulds) and exposed to UV light for $15 \mathrm{~min}\left(365 \mathrm{~nm}, 5 \mathrm{~mW} / \mathrm{cm}^{2}\right)$ to perform the crosslinking. Alginate - GelMA IPN hydrogels were prepared by combining alginate solution (DMEM, 7\% w/v) with GelMA solution of various concentrations (DMEM, 10, 20 and $30 \% \mathrm{w} / \mathrm{v}$ ) containing $0.05 \% \mathrm{w} / \mathrm{v}$ Irgacure in $1: 1$ ratio. The final mixture was poured into the same agarose- $\mathrm{CaCl}_{2}$ moulds used for alginate constructs and cured with $45 \mathrm{mM} \mathrm{CaCl}_{2}$ and under UV light exposure for 20 and 15 min respectively.

\subsection{Isolation and expansion of MSCs and CCs}

Bone marrow was removed from the femoral shaft of a porcine donor and washed in high-glucose Dulbecco's Modified Eagle Medium (hgDMEM) (Biosciences) supplemented with $10 \%$ foetal bovine serum (FBS), $1 \%$ penicillin $(100 \mathrm{U} / \mathrm{mL})$ - streptomycin $(100 \mu \mathrm{g} / \mathrm{mL})$ (all Bioscience) and amphotericin B $(2.5 \mu \mathrm{g} / \mathrm{mL})$ (SigmaAldrich). A homogenous cell suspension was achieved by triturating with a $18 \mathrm{G}$ needle. The solution was centrifuged twice at $650 \mathrm{~g}$ for $5 \mathrm{~min}$, with removal of the supernatant. The resultant cell pellet was triturated, and the cell suspension was filtered through a $40 \mu \mathrm{m}$ cell sieve (Sarstedt). Cell counting was performed with trypan blue and acetic acid before plating at a density of $5 \times 103$ cells $/ \mathrm{cm}_{2}$ and maintained in a humidified chamber. Following colony formation, cells were trypsinized, counted and re-plated for a further passage at a density of $5 \mathrm{x}$ 103 cells/cm2. FGF-2 (Prospec-Tany TechnoGene Ltd) was added to the media which was changed twice weekly.

Cartilage tissue from another porcine donor was rinsed with PBS, weighed and finely diced. Chondrocytes were isolated by digestion in hgDMEM containing $1 \%$ penicillin (100 $\mathrm{U} / \mathrm{mL})$ - streptomycin $(100 \mu \mathrm{g} / \mathrm{mL})$ and $8 \mathrm{~mL} / \mathrm{g}$ of collagenase type II $(350 \mathrm{U} / \mathrm{mL})$ for $12-14 \mathrm{~h}$ under constant rotation at 37 ${ }^{\circ} \mathrm{C}$. The resulting cell suspension was filtered through a $40 \mu \mathrm{m}$ cell sieve, centrifuged and rinsed with PBS twice. Cell number and viability were determined using a haemocytometer and $0.4 \%$ trypan blue staining.

All expansion was conducted in low oxygen conditions $\left(5 \% \mathrm{pO}_{2}\right)$ and media was changed twice weekly.

\subsection{D bioprinting process}

All 3D printed constructs were fabricated using the 3D Discovery bioplotter purchased from RegenHU with spatial resolution of $\pm 5 \mu \mathrm{m}$. Porous PCL (CAPA 6500D, Perstorp, Mn $50 \mathrm{kDa}$ ) scaffolds with two different geometries named Aligned and Double Offset were printed using a FDM printhead. All printing parameters are described in Table 1.

For the in vitro evaluation, previously printed PCL scaffolds were sterilized with ethylene oxide (EtO). Prior to hydrogels printing, the PCL constructs were placed in each well of a 6-well plate by means of 3D printed poly lactic acid inserts containing PDMS moulds that kept the scaffolds in place in the centre of the well. This allowed for $\mathrm{Z}$-direction printing of the hydrogel bioinks through microextrusion bioprinting. 
Table 1. Summary of FDM and microextrusion printing parameters for PCL and hydrogels.

\section{Printing Parameters $\quad$ FDM printing Microextrusion printing}

\begin{tabular}{|c|c|c|c|c|}
\hline Material & PCL & $3.5 \%$ alginate & $5 \%$ gelMA & IPN \\
\hline Needle (Gauge) & 30 & 23 & 23 & 23 \\
\hline Pressure (bar) & 1 & 0.5 & 0.3 & 1.3 \\
\hline Screw Speed (revs/m) & 10 & - & - & - \\
\hline Temperature $\left({ }^{\circ} \mathrm{C}\right)$ & $86($ tank), 78 (needle) & - & 13 & 13 \\
\hline Layer Thickness (mm) & 0.1 & - & - & - \\
\hline Extrusion Speed $(\mathrm{mm} / \mathrm{s})$ & 6 & 4 & 2 & 4 \\
\hline
\end{tabular}

To form the single hydrogel component bioinks, alginate and GelMA were dissolved at a concentration of 7 and $10 \%$ w/v respectively. To produce the IPN bioink, alginate and GelMA were firstly dissolved to 14 and $20 \%$ w/v respectively and then mixed together in 1:1 ratio using a luer lock system (7 and 10\% w/v alginate and GelMA final respectively). As co-cultures of BMSCs and CCs have been shown to support stable cartilage tissue formation resistant to hypertrophy and endochondral ossification in vivo [64], using the above mentioned luer lock system, the obtained bioinks were then combined with a mixture of BMSCs and CCs (3:1 ratio) in 1:1 ratio $(3.5 \% \mathrm{w} / \mathrm{v}$ alginate final, $5 \% \mathrm{w} / \mathrm{v}$ GelMA final, $3.5 \% \mathrm{w} / \mathrm{v}$ alginate $-5 \%$ w/v GelMA IPN final, 20 x 106 cells $/ \mathrm{mL})$. Cellencapsulated bioinks were loaded into a syringe and the plunger was moved up and down until air bubbles were collected at the tip of the syringe and could be removed. After minimizing the presence of air bubbles, individual hydrogel components were mixed using a dual syringe approach to form the IPN.

Using a z-direction bioprinting approach, the pores of the previously printed PCL networks were loaded with either alginate, GelMA or alginate-GelMA IPN bioinks. Z-direction bioprinting involved using a generated custom G-code to deposit the required bioink by moving the needle in the $\mathrm{z}$ direction through the open pores of the PCL networks. To make sure that cell-encapsulated bioinks were homogeneously distributed, the PCL networks were soaked in $45 \mathrm{mM} \mathrm{CaCl}_{2}$ and let rotate overnight before bioprinting. This initiated the crosslinking process of the bioinks as soon as they were extruded, avoiding settling of the hydrogels at the bottom of the PCL scaffolds as well as formation of empty spaces. See Table 2 for printing parameters. Following the fabrication process, the constructs were fully crosslinked as follows: PCL + alginate scaffolds were immersed in a bath of $45 \mathrm{mM} \mathrm{CaCl}_{2}$ for 20 mins; PCL + GelMA constructs underwent UV light exposure for 15 mins; PCL + IPN constructs were subjected to both $\mathrm{CaCl}_{2}(45 \mathrm{mM})$ and UV light crosslinking for 15 and 20 min respectively.

\subsection{In vitro culture conditions}

3D bioprinted scaffolds were cultured for 6 weeks in chondrogenically defined media consisting of hgDMEM supplemented with $1 \%$ penicillin $(100 \mathrm{U} / \mathrm{mL})$ - streptomycin $(100 \mu \mathrm{g} / \mathrm{mL})$, sodium pyruvate $(100 \mu \mathrm{g} / \mathrm{mL})$, L-proline $(40$ $\mu \mathrm{g} / \mathrm{mL}), \mathrm{L}$-ascorbic acid 2-phosphate $(50 \mu \mathrm{g} / \mathrm{mL})$, linoleic acid $(4.7 \mu \mathrm{g} / \mathrm{mL})$, bovine serum albumin $(1.5 \mathrm{mg} / \mathrm{mL}), 1 \mathrm{x}$ insulin-transferrin-selenium, dexamethasone (100 nM) (all Sigma-Aldrich) and human TGF-B3 (10 ng/mL) (ProspecTany TechnoGene Ltd). Media change was performed twice weekly. To test whether a co-culture of MSCs and CCs within a bioink is capable of promoting the development of phenotypically stable articular cartilage, constructs were maintained at $5 \% \mathrm{pO} 2$ for the first 3 weeks of culture to induce robust chondrogenesis, and then switched to $20 \% \mathrm{pO} 2$ for the final 3 weeks to test the capacity of the co-culture to maintain a stable cartilage phenotype.

\subsection{Live/Dead confocal microscopy}

Cell viability was evaluated $24 \mathrm{~h}$ post-printing using a Live/Dead assay kit (Bioscience). Bioprinted cell-laden scaffolds were rinsed with phenol free medium and incubated in a solution containing $4 \mu \mathrm{M}$ ethidium homodimer- 1 and 2 $\mu \mathrm{M}$ calcein for $1 \mathrm{~h}$. Following incubation, the scaffolds were rinsed again and imaged with Olympus FV-1000 PointScanning Confocal Microscope t 488 and $543 \mathrm{~nm}$ channels. Cell viability was quantified using Image-J software. 


\subsection{Biochemical analysis}

The biochemical content of all constructs was quantified. Samples were digested with papain $(125 \mu \mathrm{g} / \mathrm{mL})$ in $0.1 \mathrm{M}$ sodium acetate, $5 \mathrm{mM}$ L-cysteine- $\mathrm{HCl}, 0.05 \mathrm{M}$ ethylenediamine-tetracetic acid (EDTA) (all Sigma-Aldrich) and $\mathrm{pH} 6$ under constant rotation at $60{ }^{\circ} \mathrm{C}$ for $18 \mathrm{~h}$. DNA content was assessed using the Hoechst Bisbenzimide 33258 dye assay. The amount of sulphated glycosaminoglycans (sGAG) was measured using the dimethyl methylene blue-binding assay (DMMB) (Blyscan, Bicolor Ltd.). Total collagen content was determined by quantifying the hydroxyproline content using the (dimethylamino)benzaldehyde and chloramine $\mathrm{T}$ assay and a hydroxyproline to collagen ratio of 1:7.69. As the gelatin within the GelMA group is derived from collagenous material, day 1 data is not included in relevant figures because the hydroxyproline assay detects the gelatin in the GelMA day 1 samples. Therefore, day 1 data is subtracted from the day 21 and day 42 data for all hydrogel groups to determine the actual produced collagen during in vitro culture.

\subsection{Histological and Immunohistochemical analysis}

Scaffolds were fixed with $4 \%$ paraformaldehyde solution, dehydrated in graded series of ethanol, embedded in paraffin wax, sectioned at $8 \mu \mathrm{m}$ and affixed to microscope slides. The sections were stained with Alcian Blue/Aldehyde Fuchsin, Picrosirius Red and Alizarin Red to assess for sGAG, collagen and calcium content.

Immunohistochemical technique was used to evaluate collagen types II and X. Sections were rehydrated and treated with chondroitinase ABC (Sigma-Aldrich) in a humidified environment at $37{ }^{\circ} \mathrm{C}$ to enhance permeability of the extracellular matrix. This was followed by incubation in goat serum to block non-specific sites and the relevant collagen type II (sc52658, 1:400) (mouse monoclonal; Santa Cruz Biotechnology) or collagen type X (ab49945, 1:200) primary antibodies (mouse monoclonal; Abcam) were applied overnight at $4{ }^{\circ} \mathrm{C}$. Treatment with peroxidase preceded the application of the secondary antibody (collagen type II, B7151, 1:300; collagen type $\mathrm{X}$, ab49760, 1:200) at room temperature for $1 \mathrm{~h}$. Thereafter, all sections were incubated with ABC reagent (Vectastain PK-400; Vector Labs) for 45 min. Finally, sections were developed with DAB peroxidase (Vector Labs) for $5 \mathrm{~min}$. Positive and negative controls were included in the immunohistochemical staining protocols.

\subsection{Mechanical characterization}

All mechanical experiments were performed at room temperature $\left(\sim 25^{\circ} \mathrm{C}\right)$ using a twin column Zwick universal testing machine (Zwick, Roell). Unconfined compression tests were carried out in a PBS bath using a $100 \mathrm{~N}$ load cell. To ensure contact between the surface of the scaffolds $(n=4$ per group) and the top compression platen, a preload of $0.05 \mathrm{~N}$ was used for hydrogels alone constructs $(2 \mathrm{~mm}$ high $\times 6 \mathrm{~mm}$ diameter), whereas $0.5 \mathrm{~N}$ preload was applied to PCL alone as well as composite constructs ( $9 \mathrm{~mm} \times 9 \mathrm{~mm} \times 4 \mathrm{~mm}$ ). Hydrogels alone constructs were produced in the shape of a cylinder (A combined stress-relaxation and dynamic compression protocol (Supplementary Figure 1a) was implemented, where a series of compressive strains were applied in increasing steps of $10 \%$ to a maximum of $30 \%$. Peak strain was reached within $500 \mathrm{~s}$ and the equilibrium stress was obtained after a relaxation time of $45 \mathrm{~min}$. After the relaxation phase, five compressive cycles at $1 \%$ strain at a frequency of $1 \mathrm{~Hz}$ were superimposed. Ramp (or compressive), equilibrium and dynamic moduli were quantified at each strain increment. The ramp modulus was calculated as the slope of the initial linear region of the obtained stress-strain curves. The equilibrium modulus was determined as the equilibrium force divided by the sample's cross-sectional area divided by the applied strain. The dynamic modulus was measured as the average force amplitude over the five cycles divided by the sample's crosssectional area divided by the applied strain amplitude.

Cell-laden 3D bioprinted constructs underwent a shorter version of the stress-relaxation and dynamic compression protocol, to prevent cell death as there was no temperature and/or oxygen control during testing. Samples $(n=3)$ were subjected to $20 \%$ compressive strain followed by $45 \mathrm{~min}$ relaxation time and five dynamic compressive cycles at $1 \%$ strain and $1 \mathrm{~Hz}$ frequency. Ramp, equilibrium and dynamic moduli were quantified as explained in the previous paragraph.

Uniaxial tensile tests on 3D printed dog bone-shaped PCL samples were performed. Each sample ( $n=4$ per group) was mounted on a sandpaper frame with Araldite glue (Huntsman). The sandpaper frame, along with the PCL scaffold attached to it, was then fixed between two grips. Every test started after cutting through both edges of the sandpaper frame to allow for stretching of the sample. Tensile specimens were tested using a $2500 \mathrm{~N}$ load cell at a displacement rate of $1 \mathrm{~mm} / \mathrm{min}$ and loaded to $50 \%$ strain. The tensile modulus was taken as the slope of the stress-strain plots between 2 and $7 \%$ strain.

\subsection{Finite Element Analysis}

IPN only, PCL only and composite constructs $(4.5 \mathrm{~mm} \mathrm{x}$ $4.5 \mathrm{~mm} \times 4 \mathrm{~mm}$ ) were modelled using axisymmetric $\mathrm{FE}$ models developed in Abaqus 6.14 (DS Simulia, USA) to evaluate von Mises stress, maximum principal stress, hydrostatic pressure and scaffold lateral expansion upon loading application. PCL structures were modelled according to a previously described approach [65]. PCL reinforced IPN constructs were simulated by placing an IPN hydrogel matrix inside the pores of the PCL scaffold. A tie interaction between the PCL and IPN meshes was applied in order to constrain the translation degrees of freedom of the common nodes of the 
IPN to those of the PCL scaffold. The models simulated ramp compression tests in unconfined configuration, until $10 \%$ strain was applied. For all groups, the nodes at the bottom ends of the scaffolds were constrained only in the loading direction, allowing for scaffold lateral expansion in the other two directions. A displacement of approximately $0.4 \mathrm{~mm}$ was prescribed through a platen that was considered a rigid body and in full contact with the nodes at the top surface of the scaffolds. Symmetric boundary conditions were applied to the constructs' inner faces.

Table 2. Material parameters for the PCL and IPN hydrogel used for the numerical analysis.

\section{Material Material Model Material Properties}

\begin{tabular}{|c|c|c|}
\hline PCL & Isotropic elastic & $\mathrm{E}=430 \mathrm{MPa} ; v=0.3$ \\
& Isotropic plastic & $\begin{array}{c}\sigma_{\text {true }}^{\mathrm{y}}=17.745 \mathrm{MPa} ; \varepsilon_{\text {true }}^{\mathrm{y}}=0 ; \\
\sigma_{\text {true }}^{\mathrm{f}}=113.39 \mathrm{MPa} ; \varepsilon_{\text {true }}^{\mathrm{f}}=1.3316\end{array}$ \\
\hline IPN & Isotropic elastic & $\mathrm{E}=0.036 \mathrm{MPa} ; v=0.49$ \\
\hline
\end{tabular}

The engineering compressive modulus of the constructs was obtained from the stress and strain values of the linear region of the curve, calculated from the displacement and resultant reaction force data computed from the simulations. Quadratic ten-node tetrahedral elements (C3D10) were used. Material properties were determined from experimental mechanical testing. Isotropic elastic behaviour was assumed for the IPN hydrogel with a Poisson's ratio of 0.49 , representing an almost incompressible material [66]. Based on our previous work [65], isotropic elastic and plastic material properties were assigned to the PCL. Material properties of both PCL and IPN are summarized in Table 2.

\subsection{Statistical analysis}

Results are presented as mean \pm standard deviation. Statistical analysis was performed with GraphPad (GraphPad Software, La Jolla California, USA). Experimental groups were analysed for significant differences using either a t-test or a general linear model for analysis of variance (ANOVA) and preforming Tukey's post-test. For all comparisons, significance was accepted for $\mathrm{p} \leq 0.05$.

\section{Results and discussion}

\subsection{Alginate - GelMA IPNs possessed superior mechanical properties to their single hydrogel components}

The mechanical properties of alginate - GelMA IPN hydrogels were first compared to that of the individual hydrogel components. This was achieved using a series of stress-relaxation tests combined with dynamic tests in an unconfined compression configuration (Supplementary Figure 1a). The ramp phase of the unconfined compression test clearly showed that the IPN hydrogel was significantly stiffer than either the alginate or GelMA hydrogel alone (Supplementary Figure 1b). From the ramp phase of each compression test (to either $10 \%, 20 \%$ or $30 \%$ strain), it was possible to quantify the ramp modulus of the hydrogels (Figure 1a; Supplementary Figure 2 a-c; Table 3). The ramp modulus of IPN hydrogel was significantly higher than the alginate and GelMA only hydrogels, and significantly higher than the sum of the individual hydrogel components (shown by the red dashed line) at $20 \%$ and $30 \%$ strain, demonstrating a synergistic increase in stiffness. Strain stiffening was observed for all three hydrogels, with the ramp modulus increasing with the applied strain amplitude.

Equilibrium and dynamic properties are other important functional parameters to measure when assessing articular cartilage and biomaterials designed to regenerate this tissue [1, $3,5,67]$. Stress-relaxation tests were next used to determine the equilibrium modulus of the hydrogels in the absence of fluid flow. Stress-time curves (Supplementary Figure 1c) revealed the distinct stress-relaxation behaviour of each hydrogel. Alginate gels exhibited a complete relaxation of the stress after only $900 \mathrm{~s}$, presumably due to the unbinding of the ionic crosslinker followed by polymer matrix flow [68-71]. In contrast, GelMA hydrogels displayed relatively little stress 
cartilage is associated with the low permeability of the solid matrix $[3,4]$.

In spite of the improvements associated with the IPN hydrogel, its equilibrium and dynamic mechanical properties still remained at least one order of magnitude lower than that of native articular cartilage (equilibrium modulus ranges from approximately 0.2 to $2 \mathrm{MPa}$, while the dynamic modulus ranges from approximately 10 to $60 \mathrm{MPa})[15,72-74]$.

Table 3. Summary of constructs mechanical properties under unconfined compression.

\begin{tabular}{|c|c|c|c|c|c|c|c|c|c|}
\hline & \multicolumn{3}{|c|}{$\begin{array}{c}\text { Ramp Modulus } \\
\text { (MPa) }\end{array}$} & \multicolumn{3}{|c|}{$\begin{array}{l}\text { Equilibrium Modulus } \\
\text { (MPa) }\end{array}$} & \multicolumn{3}{|c|}{$\begin{array}{c}\text { Dynamic Modulus } \\
\text { (MPa) }\end{array}$} \\
\hline & $\begin{array}{l}10 \% \\
\text { strain }\end{array}$ & $\begin{array}{c}20 \% \\
\text { strain }\end{array}$ & $\begin{array}{l}30 \% \\
\text { strain }\end{array}$ & $\begin{array}{l}10 \% \\
\text { strain }\end{array}$ & $\begin{array}{l}20 \% \\
\text { strain }\end{array}$ & $\begin{array}{c}30 \% \\
\text { strain }\end{array}$ & $\begin{array}{l}10 \% \\
\text { strain }\end{array}$ & $\begin{array}{l}20 \% \\
\text { strain }\end{array}$ & $\begin{array}{l}30 \% \\
\text { strain }\end{array}$ \\
\hline $3.5 \%$ Alginate & $\begin{array}{c}0.011 \pm \\
0.003\end{array}$ & $\begin{array}{c}0.044 \pm \\
0.031\end{array}$ & $\begin{array}{c}0.049 \pm \\
0.026\end{array}$ & $\begin{array}{c}0.001 \pm \\
0.001\end{array}$ & $\begin{array}{c}0.006 \pm \\
0.005\end{array}$ & $\begin{array}{c}0.007 \pm \\
0.007\end{array}$ & $\begin{array}{c}0.046 \pm \\
0.034\end{array}$ & $\begin{array}{c}0.066 \pm \\
0.058\end{array}$ & $\begin{array}{c}0.094 \pm \\
0.095\end{array}$ \\
\hline $5 \%$ GelMA & $\begin{array}{c}0.008 \pm \\
0.001\end{array}$ & $\begin{array}{c}0.015 \pm \\
0.003\end{array}$ & $\begin{array}{c}0.018 \pm \\
0.002\end{array}$ & $\begin{array}{c}0.005 \pm \\
0.002\end{array}$ & $\begin{array}{c}0.005 \pm \\
0.003\end{array}$ & $\begin{array}{c}0.007 \pm \\
0.002\end{array}$ & $\begin{array}{c}0.015 \pm \\
0.006\end{array}$ & $\begin{array}{c}0.018 \pm \\
0.005\end{array}$ & $\begin{array}{c}0.027 \pm \\
0.005\end{array}$ \\
\hline$I P N$ & $\begin{array}{c}0.036 \pm \\
0.009\end{array}$ & $\begin{array}{c}0.110 \pm \\
0.035\end{array}$ & $\begin{array}{c}0.214 \pm \\
0.048\end{array}$ & $\begin{array}{c}0.013 \pm \\
0.011\end{array}$ & $\begin{array}{c}0.028 \pm \\
0.005\end{array}$ & $\begin{array}{c}0.034 \pm \\
0.001\end{array}$ & $\begin{array}{c}0.147 \pm \\
0.044\end{array}$ & $\begin{array}{c}0.265 \pm \\
0.070\end{array}$ & $\begin{array}{c}0.385 \pm \\
0.090\end{array}$ \\
\hline $\begin{array}{c}\text { Double Offset } \\
\text { PCL }\end{array}$ & $\begin{array}{c}0.221 \pm \\
0.060\end{array}$ & $\begin{array}{c}0.304 \pm \\
0.041\end{array}$ & $\begin{array}{c}0.623 \pm \\
0.092\end{array}$ & $\begin{array}{c}0.246 \pm \\
0.041\end{array}$ & $\begin{array}{c}0.261 \pm \\
0.043\end{array}$ & $\begin{array}{c}0.395 \pm \\
0.036\end{array}$ & $\begin{array}{c}0.515 \pm \\
0.127\end{array}$ & $\begin{array}{c}0.777 \pm \\
0.117\end{array}$ & $\begin{array}{c}1.603 \pm \\
0.205\end{array}$ \\
\hline $\begin{array}{c}\text { Double Offset } \\
\text { PCL }+I P N\end{array}$ & $\begin{array}{c}0.775 \pm \\
0.081\end{array}$ & $\begin{array}{c}1.034 \pm \\
0.378\end{array}$ & $\begin{array}{c}1.445 \pm \\
0.256\end{array}$ & $\begin{array}{c}0.203 \pm \\
0.215\end{array}$ & $\begin{array}{c}0.652 \pm \\
0.284\end{array}$ & $\begin{array}{c}0.957 \pm \\
0.527\end{array}$ & $\begin{array}{c}0.757 \pm \\
0.195\end{array}$ & $\begin{array}{c}1.415 \pm \\
0.285\end{array}$ & $\begin{array}{c}2.293 \pm \\
0.352\end{array}$ \\
\hline
\end{tabular}

a

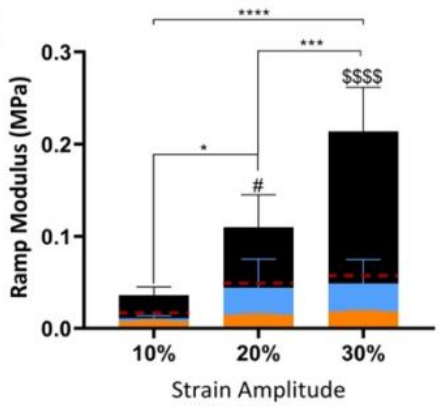

$3.5 \%$ Alginate b

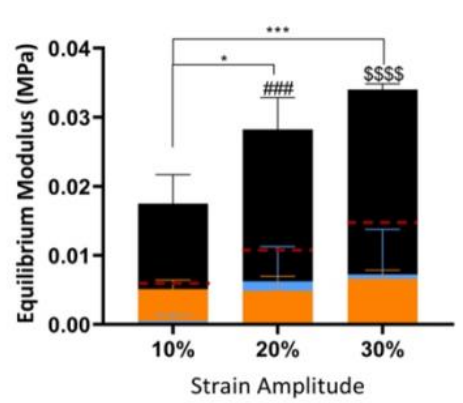

C

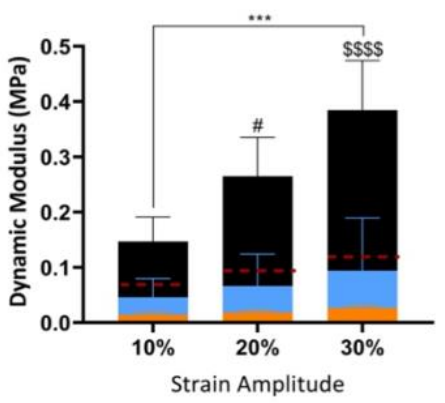

$5 \%$ GeIMA

Figure 1. (a) Ramp modulus, (b) equilibrium modulus and (c) dynamic modulus in unconfined compression of $3.5 \%$ alginate (blue bars), 5\% GelMA (orange bars) and IPN (black bars) hydrogels when applying increasing levels of strain amplitude: 10, 
Table 4. Summary of compressive and tensile properties for Aligned and Double Offset PCL.

\subsection{D printing of $P C L$ networks with tension- compression nonlinearity}

Recognizing that hydrogels alone [43, 75-79], even IPN hydrogels, fail to recapitulate the complex mechanical properties of articular cartilage, we next sought to investigate whether it was possible to design more biomimetic materials with nonlinear mechanical properties by reinforcing IPN hydrogels with 3D printed PCL networks with distinct tension-compression nonlinearity.

\begin{tabular}{|c|c|c|}
\hline & $\begin{array}{c}\text { Compressive Modulus } \\
(\mathbf{M P a})\end{array}$ & $\begin{array}{c}\text { Tensile Modulus } \\
(\mathbf{M P a})\end{array}$ \\
\hline Aligned PCL & $1.341 \pm 0.095$ & $14.795 \pm 0.278$ \\
\hline Double Offset $P C L$ & $0.218 \pm 0.014$ & $8.358 \pm 0.990$ \\
\hline
\end{tabular}

a Aligned
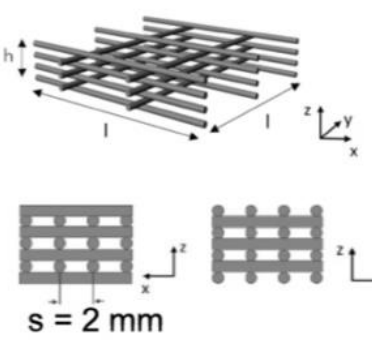

C

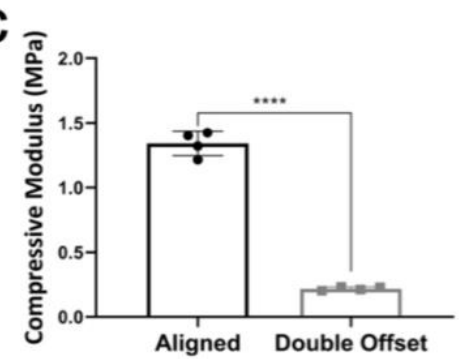

Double Offset
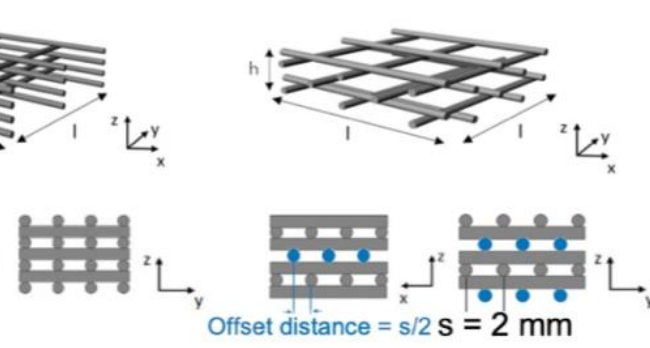

d

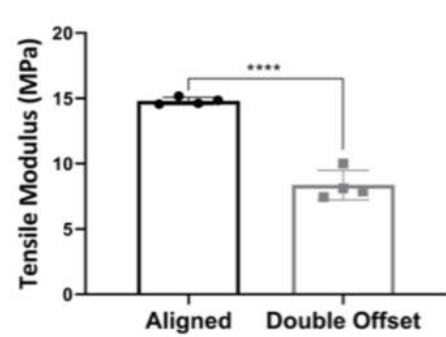

b Aligned Double Offset

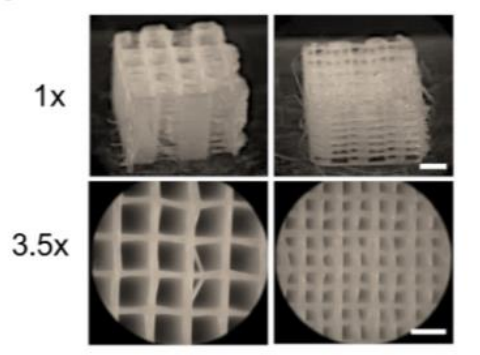

e

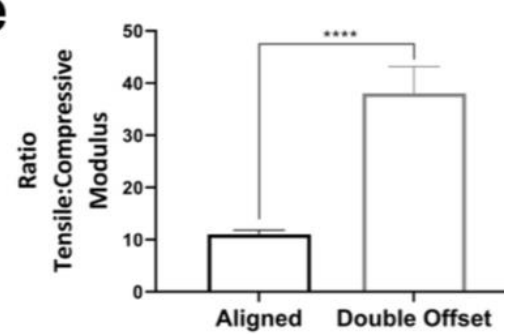

Figure 2. (a) Schematic of the PCL scaffolds depicting the Aligned geometry characterized by a regular orthogonal architecture and the Double Offset geometry comprising of offset layers present in both xz and yz planes. Offset layers are shown in blue. Scaffold architecture features are also indicated: $h$, height of the scaffold; l, length of the scaffold; s, fibre spacing. (b) Microscopy images of Aligned (left column) and Double Offset (right column) constructs. Scale bar: 2 mm. (c) Tensile modulus, (d) compressive modulus and (e) ratio between tensile and compressive modulus of Aligned (black bars) and Double Offset (grey bars) 3D printed PCL scaffolds. ${ }^{* * *} \mathrm{p}<0.0001 ; \mathrm{n}=4$ per group. 
To this end, 3D printing parameters (Table 1) were varied in order to obtain two distinct 3D PCL geometries named Aligned and Double Offset (Figure 2a, b). The Aligned geometry (Figure 2a) was characterized by a regular orthogonal architecture in a lay-down pattern of $0^{\circ} 00^{\circ} / 90^{\circ} 90^{\circ}$ forming square pores. The Double Offset geometry (Figure 2a) consisted of an orthogonal architecture with the same laydown pattern. This differed from the Aligned pattern because of the presence of offset layers every other four layers that were deposited at an offset distance equal to half the fibre spacing. The obtained 3D network/scaffold architectures were tested in uniaxial tension and compression configurations to evaluate the effect of network geometry on the degree of tension-compression nonlinearity. Aligned constructs were stiffer in both tension and compression compared to Double Offset networks (Figure 2c, d; Table 4), with more dramatic differences observed in the compressive properties of the PCL networks. The compressive modulus was $1.341 \pm 0.095$ and $0.218 \pm 0.014 \mathrm{MPa}$ for Aligned and Double Offset samples respectively (Figure 2c; Table 4), whereas he tensile stiffness was $14.795 \pm 0.278 \mathrm{MPa}$ for the Aligned geometry and 8.358 $\pm 0.990 \mathrm{MPa}$ for the Double Offset one (Figure 2d; Table 4). When quantifying the ratio between tensile and compressive moduli, higher tension-compression nonlinearity was observed in the Double Offset networks in comparison to Aligned samples (Figure 2e).

\subsection{Combining IPN hydrogels with reinforcing PCL networks to produce biomimetic composites with mechanical properties comparable to native cartilage}

As tension-compression nonlinearity is believed to play a critical role in the load-bearing capacity of articular cartilage by enhancing fluid pressurization under compression, we hypothesized that reinforcing IPN hydrogels with a Double Offset PCL network design would result in the development of constructs that more closely mimicked the mechanical behaviour of the native tissue. To test this hypothesis, we next reinforced the alginate - GelMA IPNs with 3D printed PCL networks with two distinct architectures (Aligned and Double Offset).

a
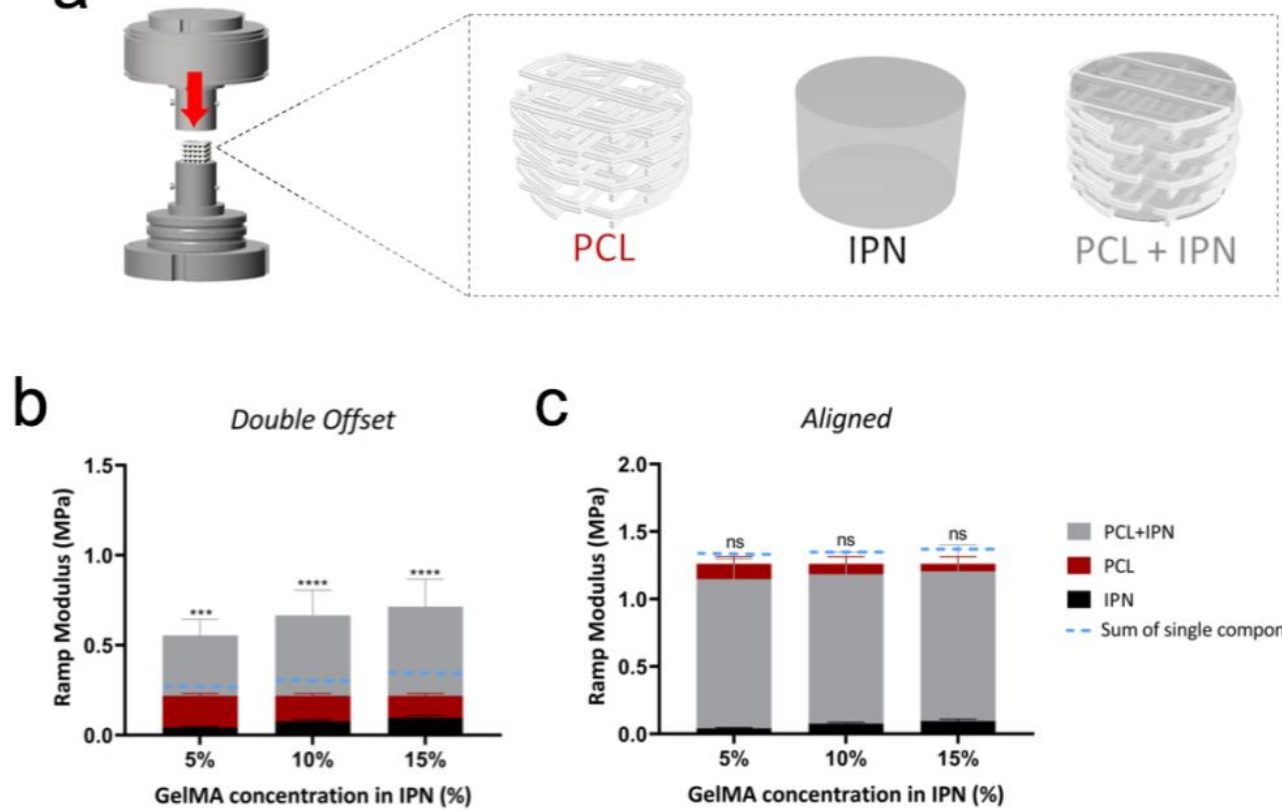
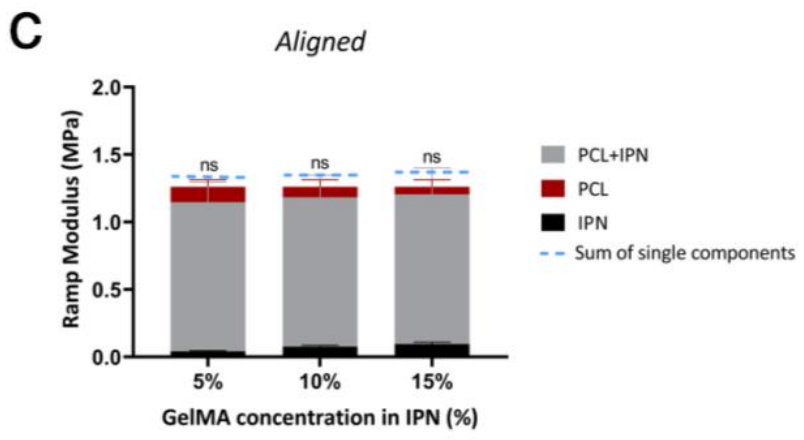

Figure 3. (a) Schematic illustration of the mechanical testing set-up and scaffold groups (PCL, IPN and PCL + IPN) that underwent compression tests. (b,c) Ramp modulus of PCL frames (red bars), IPN hydrogels (black bars) and PCL + IPN scaffolds (grey bars) when varying GeIMA concentration in the IPN from 5 to $15 \%(\mathrm{w} / \mathrm{v})$. Results are shown for groups containing either the (b) Aligned or (c) Double Offset PCL geometry. The sum of the compressive modulus of the individual components for each GeIMA concentration is indicated by the dashed blue line. ${ }^{* * *} p<0.001$ and ${ }^{* * * *} p<0.0001 ; n=4$ per group. 
a

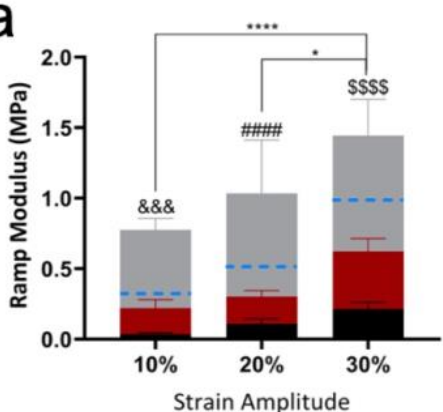

b

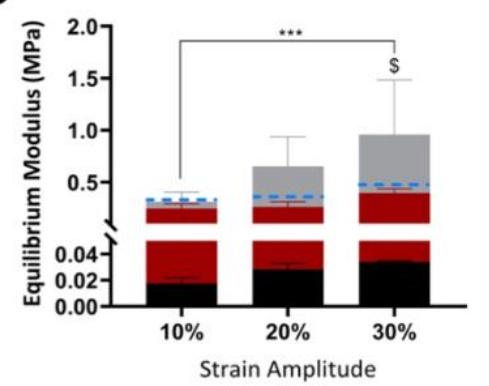

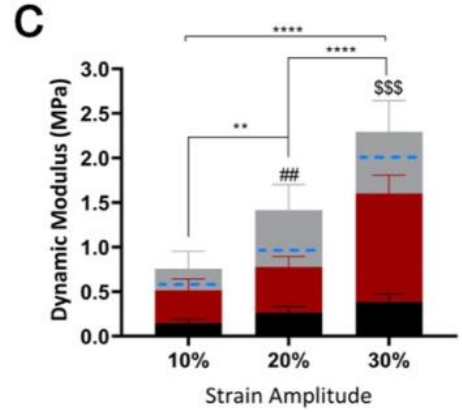

\section{IPN $\square \mathrm{PCL} \square \mathrm{PCL}+\mathrm{IPN}--$ Sum of single components}

Figure 4. (a) Ramp modulus, (b) equilibrium modulus and (c) dynamic modulus in unconfined compression of IPN hydrogel (black bars), Double Offset PCL (red bars) and PCL + IPN (grey bars) scaffolds when applying increasing levels of strain amplitude: 10,20 and $30 \%$. The sum of the moduli of the individual components for each applied strain is indicated by the dashed blue line. \&\&\&p $<0.001 \mathrm{PCL}+$ IPN vs. PCL at $10 \%$ applied strain; \#\#p $<0.01$ and \#\#\# $\mathrm{p}<0.0001 \mathrm{PCL}+$ IPN vs. PCL at $20 \%$ applied strain; ${ }^{\$} p<0.05, \$ \$ p<0.001$ and $\$ \$ \$ p<0.0001 \mathrm{PCL}+\mathrm{IPN}$ vs. PCL at $30 \%$ applied strain; ${ }^{*} \mathrm{p}<0.05,{ }^{* *} \mathrm{p}<0.01,{ }^{* * *} \mathrm{p}<0.001$ and ${ }^{* * * *} \mathrm{p}<0.0001$ vs. $\mathrm{PCL}+\mathrm{IPN} ; \mathrm{n}=4$ per group.

The PCL scaffolds were infused with different alginate GelMA IPN hydrogel formulations where the alginate concentration $(3.5 \% \mathrm{w} / \mathrm{v})$ was maintained constant, whereas the GelMA content was increased $(5,10$ and $15 \%$ w/v). The resulting composite constructs were subjected to compression tests to determine their ramp modulus in comparison to PCL scaffolds alone, IPNs alone and the sum of the moduli of the individual components (Figure 3a-c; Supplementary Figure 3). After infusing the Double Offset PCL scaffolds with the IPN hydrogels (Figure 3b), a synergistic increase in stiffness was observed for all IPN formulations. Specifically, the ramp modulus of the composite samples (grey bars) was higher than the sum of the moduli of the single components (blue dashed lines) as well as to the individual components, i.e. the PCL scaffolds (red bars) and IPN gels (black bars). The ramp modulus of the composite increased as the GelMA content in the IPN increased $(0.554 \pm 0.090,0.665 \pm 0.140$ and $0.713 \pm$ $0.153 \mathrm{MPa}$ at 5,10 and $15 \%$ w/v GelMA concentration, respectively). Moreover, Double Offset PCL + IPN constructs were significantly stiffer than the Double Offset PCL alone samples $(0.218 \pm 0.014 \mathrm{MPa})$, showing approximately $2.5-$, 3and 3.3-fold increases in stiffness at 5, 10 and $15 \% \mathrm{w} / \mathrm{v}$ GelMA concentrations, respectively. The obtained ramp modulus values for the Double Offset PCL + IPN constructs fell in the range of native tissue properties ranging from approximately 0.24 to $1.4 \mathrm{MPa}[73,80,81]$. Combining the Aligned PCL constructs with the IPN hydrogels (Figure 3c) did not result in the same synergistic increase in stiffness. The ramp moduli of the composite constructs did not increase as the GelMA content in the IPN gels increased $(1.147 \pm 0.149$, $1.183 \pm 0.164$ and $1.206 \pm 0.195 \mathrm{MPa}$ at 5,10 and $15 \% \mathrm{w} / \mathrm{v}$
GelMA concentration, respectively). Furthermore, there was no statistical difference in ramp modulus compared to the Aligned PCL alone scaffolds $(1.261 \pm 0.051 \mathrm{MPa})$, indicating that the PCL component was dominating the mechanical behaviour of the composite.

The ramp, equilibrium and dynamic moduli of the composites were also assessed after the application of 10, 20 and 30\% strain for the Double Offset PCL design (Figure 4ac; Supplementary Figure 4; Table 3). Stiffness was found to increase with increases in the applied strain amplitude. For all strain levels, the ramp moduli was significantly higher for the composite constructs (grey bars; $0.775 \pm 0.081,1.034 \pm 0.378$ and $1.445 \pm 0.256 \mathrm{MPa}$ when applying 10,20 and $30 \%$ strains respectively) compared to the PCL alone (red bars; $0.221 \pm$ $0.060,0.304 \pm 0.041$ and $0.623 \pm 0.092 \mathrm{MPa}$ at 10,20 and $30 \%$ applied strain amplitudes respectively), the IPN alone (black bars) as well as the sum of the individual components (blue dashed line) at all applied strain amplitudes (Figure 4a; Supplementary Figure 4 a-c; Table 3). The equilibrium modulus of the PCL + IPN composites was only higher than the PCL scaffolds alone at higher strain levels.

In unconfined compression, the equilibrium modulus of articular cartilage ranges from approximately 0.2 to $2 \mathrm{MPa}$ $[67,73,74,82-84]$, whereas the dynamic modulus in response to cyclic loading ranges from approximately 10 to $60 \mathrm{MPa}$, depending on the loading frequency $[15,31,73,85,86]$. The composite 3D printed PCL reinforced IPN constructs possessed equilibrium moduli that fell in the range of native cartilage equilibrium properties (Figure 4b; Supplementary Figure 4 d-f; Table 3). Although the dynamic modulus of PCL + IPN composites did not match the values of the native tissue, 
a Double Offset PCL

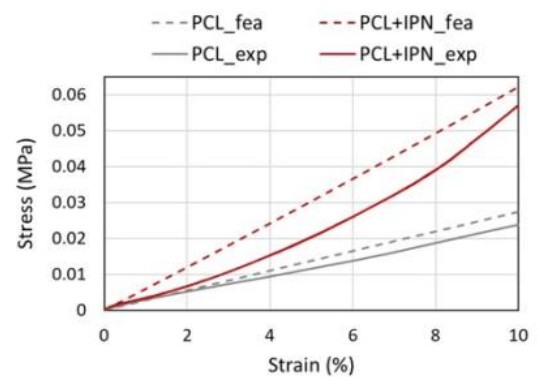

C

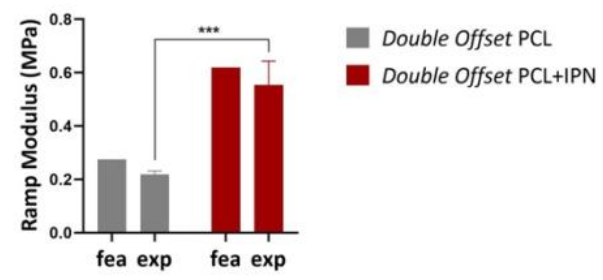

e

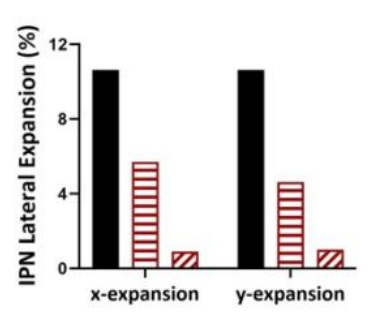

it showed a marked improvement compared to PCL and IPN alone (Figure 4c; Supplementary Figure 4 g-i; Table 3), reaching the same order of magnitude as articular cartilage. The dynamic modulus for composite constructs was measured to be $0.757 \pm 0.195,1.415 \pm 0.285$ and $2.293 \pm 0.352 \mathrm{MPa}$ at 10,20 and $30 \%$ applied strain amplitudes respectively, 政 fact its permeability is significantly higher than that of articular cartilage. While a lower permeability (and hence a superior capacity to generate fluid load support) could be achieved by increasing the density of the IPN, this could negatively impact nutrient transport and waste removal once the biomaterial is seeded with cells for tissue engineering applications. Hence, the properties achieved here can be viewed as a balance between providing initial mechanical function (mimicking native equilibrium and ramp modulus values) and an environment supportive of chondrogenesis (that is assessed below).

b Aligned PCL

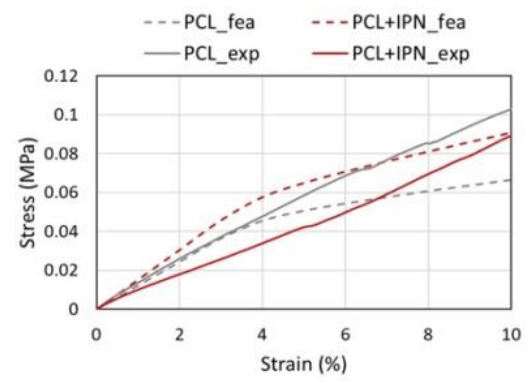

d

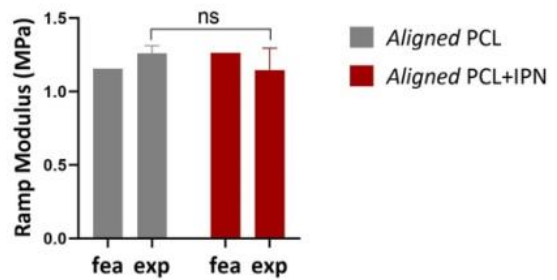

f

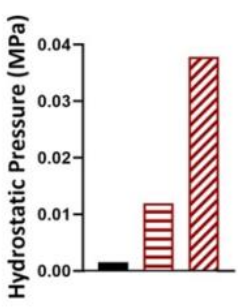

IPN alone

日 Aligned PCL+IPN

$\square$ Double Offset PCL+IPN

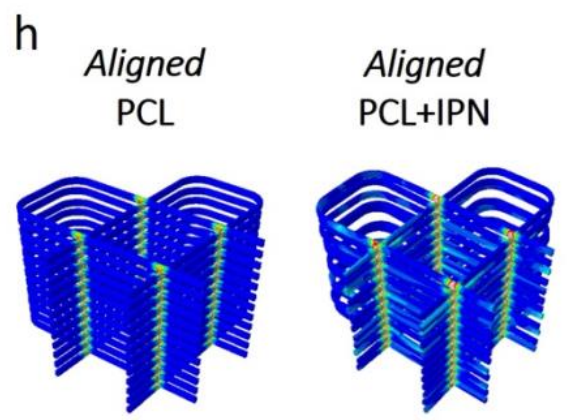

Figure 5. Computational and experimental $(\mathrm{a}, \mathrm{b})$ compressive stress-strain curves and $(\mathrm{c}, \mathrm{d})$ ramp modulus for $(\mathrm{a}, \mathrm{c})$ Double Offset and $(b, d)$ Aligned PCL-based structures; ${ }^{* * *} p<0.001 ; n=4$ per group. Comparison of predicted IPN (e) lateral expansion 
and (f) hydrostatic pressure in IPN alone, Aligned PCL+IPN and Double Offset PCL+IPN structures. Von Mises stress contour plots for (g) Double Offset and (h) Aligned PCL-based constructs.

\subsection{FE modelling to better understand the mechanism by which 3D printed fibre networks mechanically reinforce IPN hydrogels}

FEA was next utilized to better understand the mechanism by which the PCL networks were mechanically reinforcing the IPN hydrogels.

FE simulations were performed of uniaxial compression tests on the IPN alone, PCL alone and composite constructs for both Double Offset and Aligned PCL-based structures (Figure 5 ). The predicted stress-strain behaviour (Figure $5 a, b$ ) and compressive modulus (Figure 5c, d) of all groups were in good agreement with the experimental results. Having demonstrated that this computational approach was able to accurately predict the compressive mechanical behaviour of 3D printed scaffolds, we next sought to investigate how the Double Offset PCL network was reinforcing the IPN hydrogels. When articular cartilage is subjected to compressive loading, the collagen network resists lateral expansion and fluid pressurization supports a significant component of the applied load. When comparing the expansion in both x- and y-direction of IPN in Double Offset PCL + IPN, Aligned PCL + IPN and IPN alone models, it was observed that reinforcing the IPN with either of the PCL networks reduced lateral expansion of the composite, with the lowest expansion observed in the Double Offset PCL composite (Figure 5e). We next predicted the average hydrostatic pressure produced in the IPN when applying 10\% compressive strain. In general, higher pressure was generated within the IPN in the presence of both Aligned and Double Offset PCL, with the highest values predicted in Double Offset PCL + IPN structures. Compared to IPN alone, there was approximately a 24- and 7.5-fold increase in hydrostatic pressure in Double Offset PCL + IPN and Aligned PCL + IPN constructs, respectively (Figure 5f). The FE model also predicted higher stresses in the PCL filaments within the Double Offset PCL network in composites compared to Double Offset PCL only structures (Figure 5g) On the other side, little difference in von Mises stress distribution was predicted in the PCL network of the Aligned PCL + IPN composites compared to Aligned PCL only scaffolds (Figure $5 \mathrm{~h})$. Together, the FE models suggest two related mechanisms by which the Double Offset PCL network reinforces the IPN hydrogel. Firstly, the presence of the IPN in the PCL network increases the tensile stresses generated in the PCL filaments (see also Supplementary Figure 5), allowing them to play a greater role in supporting the applied load. Secondly, the PCL network is predicted to limit lateral expansion of the composite and lead to higher magnitudes of hydrostatic pressure being generated within the IPN. Such reinforcement mechanisms are enhanced by PCL networks with greater tension-compression nonlinearity.

Other studies have reported similar reinforcement mechanisms, mainly when reinforcing hydrogels with electrospun fibres. For example, Castilho et al. [66] demonstrated that the reinforcement effect of microfibre reinforced GelMA composites derived either from fibres being pulled in tension by the expansion of the hydrogel, or from the support provided by the hydrogel in preventing buckling of the fibres under compression.

The FE simulations further demonstrate that the combination of the Double Offset PCL network with the IPN produce a cartilage biomimetic construct.

\subsection{PCL reinforced cell-laden IPNs fabricated using 3D bioprinting support chondrogenesis with minimal hypertrophy}

Having successfully obtained composite constructs with mechanical properties comparable to those of native cartilage, we proceeded to assess the capacity of these composites to provide a pro-chondrogenic environment in vitro. With a view to bioprinting cell-laden implants, we established a multipletool biofabrication process that first involved the bioprinting of the porous reinforcing Double Offset PCL networks in the shape of a cylinder $(6 \mathrm{~mm} \times 6 \mathrm{~mm})$. In a second bioprinting step, the obtained PCL networks were placed in a moulding system that kept them in the centre of the well of a 6-well plate, which facilitated the $\mathrm{z}$-direction bioprinting of the bioinks (alginate, GelMA and alginate - GelMA IPNs containing a co-culture of BMSCs and CCs) into the PCL networks (Figure 6a). Z-direction bioprinting consists of depositing the cell encapsulating-bioinks within the empty pores of the previously printed PCL networks by moving the needle in the $\mathrm{z}$-direction. The bioprinted constructs were then transferred to chondrogenic media and cultured in static conditions at $5 \% \mathrm{O}_{2}$ for the first 3 weeks and then at $21 \% \mathrm{O}_{2}$ for further 3 weeks.

To verify that cell viability was not affected by the 3D printing process or the post-fabrication crosslinking of the bioinks, all constructs were analysed using live-dead staining after $24 \mathrm{~h}$ of culture (Figure $6 \mathrm{~b}-\mathrm{c}$ ). All bioinks supported high levels of cell viability ( $\sim 80 \%$; Figure $6 \mathrm{c}$ ), showing that the fabrication process was not overly harmful to the cells.

Over 6 weeks of in vitro culture, the ramp modulus of the PCL reinforced GelMA and IPN-based composites progressively increased (Figure 6d-f; Table 5). 
a

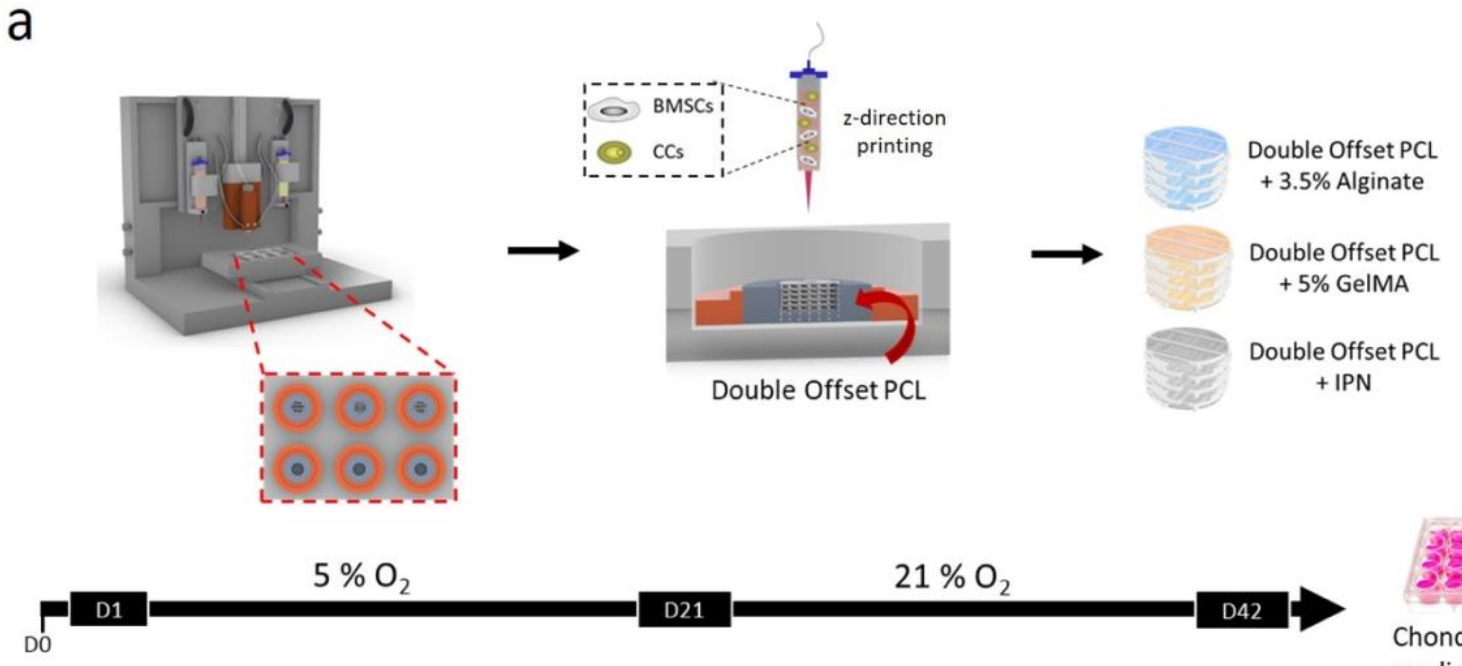

b

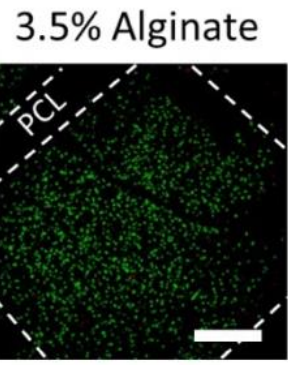

5\% GelMA

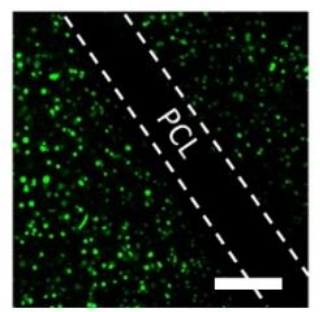

d

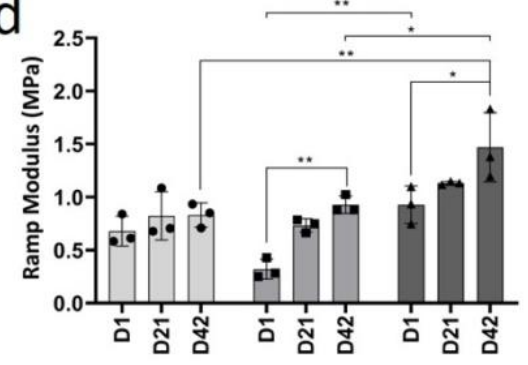

e

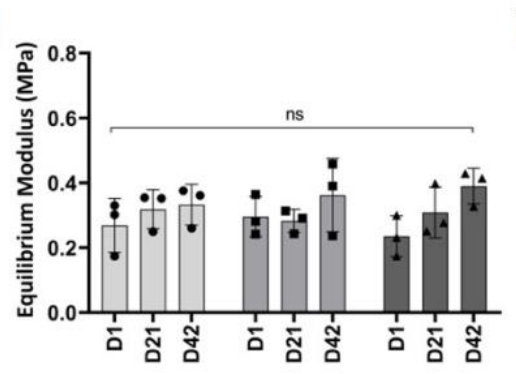

$3.5 \%$ Alginate

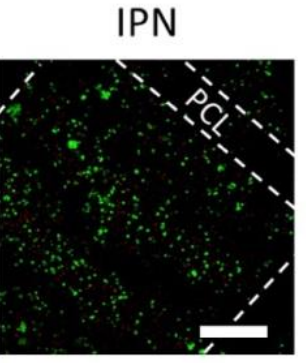

C

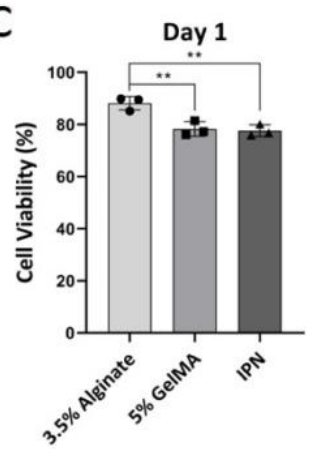

$f$

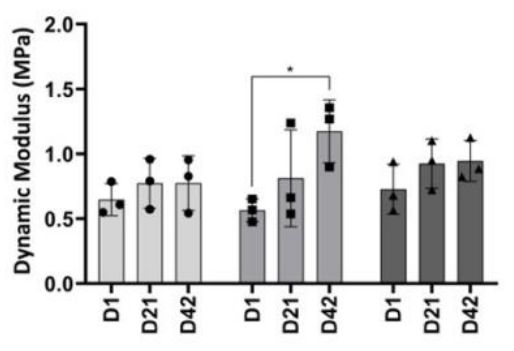

Figure 6. (a) Schematic of the experimental set-up: Double Offset PCL frames were 3D printed via FDM and then placed in a moulding system that kept the PCL scaffolds in the centre of each well of a 6-well plate to facilitate microextrusion (z-direction printing) of the cell-laden bioinks. Obtained composite constructs were cultured in chondrogenic media for the first 3 weeks in hypoxia and the following 3 weeks in normoxia. (b) Representative images of Live/Dead staining used to determine the cell viability of 3.5\% alginate, 5\% GelMA and IPN bioinks. Scale bar: $200 \mu \mathrm{m}$. (c) Quantitative analysis of the cell viability for all of the bioinks. (d) Ramp modulus, (e) equilibrium modulus and (f) dynamic modulus for all composite groups after day 1, 21 and 42 of culture. ${ }^{*} p<0.05, * * p<0.01,{ }^{* * *} p<0.001$ and ${ }^{* * *} p<0.0001 ; n=3$ per group. 
Table 5. Summary of mechanical properties of alginate-, GelMA- and IPN-based composites under unconfined compression after 1, 21 and 42 days of culture.

\begin{tabular}{|c|c|c|c|c|c|c|c|c|c|}
\hline & \multicolumn{3}{|c|}{$\begin{array}{c}\text { Ramp Modulus } \\
\text { (MPa) }\end{array}$} & \multicolumn{3}{|c|}{$\begin{array}{l}\text { Equilibrium Modulus } \\
\text { (MPa) }\end{array}$} & \multicolumn{3}{|c|}{$\begin{array}{c}\text { Dynamic Modulus } \\
\text { (MPa) }\end{array}$} \\
\hline & D1 & D21 & D42 & D1 & D21 & D42 & D1 & D21 & D42 \\
\hline $3.5 \%$ Alginate & $\begin{array}{c}0.678 \pm \\
0.141\end{array}$ & $\begin{array}{c}0.822 \pm \\
0.228\end{array}$ & $\begin{array}{c}0.830 \pm \\
0.114\end{array}$ & $\begin{array}{c}0.269 \pm \\
0.083\end{array}$ & $\begin{array}{c}0.318 \pm \\
0.060\end{array}$ & $\begin{array}{c}0.332 \pm \\
0.063\end{array}$ & $\begin{array}{c}0.647 \pm \\
0.123\end{array}$ & $\begin{array}{c}0.773 \pm \\
0.193\end{array}$ & $\begin{array}{c}0.774 \pm \\
0.211\end{array}$ \\
\hline $5 \%$ GelMA & $\begin{array}{c}0.320 \pm \\
0.094\end{array}$ & $\begin{array}{c}0.732 \pm \\
0.062\end{array}$ & $\begin{array}{c}0.927 \pm \\
0.082\end{array}$ & $\begin{array}{c}0.296 \pm \\
0.062\end{array}$ & $\begin{array}{c}0.282 \pm \\
0.036\end{array}$ & $\begin{array}{c}0.362 \pm \\
0.113\end{array}$ & $\begin{array}{c}0.565 \pm \\
0.088\end{array}$ & $\begin{array}{c}0.812 \pm \\
0.278\end{array}$ & $\begin{array}{c}1.174 \pm \\
0.345\end{array}$ \\
\hline$I P N$ & $\begin{array}{c}0.926 \pm \\
0.177\end{array}$ & $\begin{array}{c}1.133 \pm \\
0.015\end{array}$ & $\begin{array}{c}1.469 \pm \\
0.326\end{array}$ & $\begin{array}{c}0.235 \pm \\
0.063\end{array}$ & $\begin{array}{c}0.309 \pm \\
0.078\end{array}$ & $\begin{array}{c}0.390 \pm \\
0.055\end{array}$ & $\begin{array}{c}0.727 \pm \\
0.192\end{array}$ & $\begin{array}{c}0.925 \pm \\
0.190\end{array}$ & $\begin{array}{c}0.945 \pm \\
0.158\end{array}$ \\
\hline
\end{tabular}

The PCL reinforced-IPN constructs possessed the highest ramp modulus at all time points reaching values of $1.469 \pm$ $0.326 \mathrm{MPa}$ compared to $0.830 \pm 0.114$ and $0.927 \pm 0.082 \mathrm{MPa}$ for alginate-based and GelMA-based composites respectively at day 42 (Figure 6d; Table 5). No major differences in the equilibrium modulus were observed between the different groups, which was approximately $0.350 \mathrm{MPa}$ after 42 days of culture (Figure 6e; Table 5). Significant increases in dynamic modulus with time in culture were only observed in the GelMA-based composites (Figure 6f; Table 5).

All three PCL reinforced bioinks supported the development of a hyaline cartilage-like tissue that stained positive for GAG and collagen type II deposition (Figure 7ac). GAG and collagen deposition appeared quite pericellular within the IPN-based composites at day 21 (Figure 7b) but become more diffuse with further time in culture (Figure 7c). What was presumed to be non-specific type $\mathrm{X}$ collagen staining, was observed in the GelMA-based composites at day 1 , making it difficult to assess type $\mathrm{X}$ collagen deposition in this group. There was no positive staining for collagen type $\mathrm{X}$ in the other groups at any time point, suggesting that the engineered tissues were not becoming hypertrophic with time in culture. Alginate and GelMA-based scaffolds showed some positive staining for alizarin red at day 42 , demonstrating the presence of calcium deposits. In contrast, no evidence of calcium deposition was observed in IPN-based composites, suggesting then the development of phenotypically stable articular cartilage-like tissue within the IPNs. The percentage of tissue staining positive for Alizarin Red was also quantified, which demonstrated that the alginate-based constructs supported the highest levels of calcification, while calcific deposits were barely detectable in the IPN group (Figure 6g). Histological observations were confirmed by biochemical analysis, which demonstrated that GAG and collagen content significantly increased over the culture period, with no dramatic differences observed between the three hydrogel groups (Figure 7e-f).

Together these results demonstrated that alginate - GelMA IPNs were able to support robust chondrogenesis, with levels of ECM deposition comparable to that observed in the individual hydrogels. Importantly, it was shown that the increased mechanical properties achieved with the IPN did not negatively affect its biological functionality. Usually, hydrogels presenting cell binding motifs such as GelMA can facilitate cell-cell and cell-matrix interactions leading more to fibrocartilage-like tissue formation [78, 87-90]. In contrast, alginate is known to be an inert material and should facilitate MSCs to develop a round shape, which is known to support a chondrogenic phenotype [78, 91, 92]. Given that a co-culture of CCs and MSCs is known to support the generation of phenotypically stable articular cartilage [64, 93-96], we did not expect to see dramatic difference in hypertrophy and progression along an endochondral pathway in the three different hydrogel bioink groups. Importantly, the stiffer IPN based composites supported the development of cartilage resistant to calcification after 6 weeks of culture, despite the fact that stiffer hydrogel environments can be more supportive of chondrocyte hypertrophy [37, 97-99].

Although the architecture of the 3D printed PCL networks did not mimic the arrangement of the collagen fibres in the native articular cartilage, the findings of this study show that the internal fibre pattern of such scaffolds can be tailored to ultimately engineer constructs with key mechanical properties (i.e. nonlinearity, equilibrium and dynamic properties) that match or approach those of cartilage prior to any in vitro culture, making them potentially suitable for immediate implantation. In addition, designing PCL networks with an offset pattern results in relatively high tension-compression 
nonlinearity, which, similarly to the collagen network in the native tissue, limits lateral expansion of the constructs and enhances the hydrostatic pressure within the IPN during compressive loading. Furthermore, the PCL + IPN composites support robust chondrogenesis, ensuring that the mechanical properties of cell-laden constructs will continue to improve (e.g. with time in culture) as the encapsulated cells secrete a cartilage-like ECM.

As the architecture of the collagenous network is known to playing a key role in determining the load-bearing properties of soft tissues such as articular cartilage [82], future studies could use 3D bioprinting strategies to develop scaffolds that also replicate specific structural characteristics of cartilage. Specifically, 3D bioprinting could potentially enable the production of either polymer networks that mimic the zonespecific arrangement of the collagen fibres in the native tissue, and/or polymeric fibre networks that could direct the alignment of the newly formed matrix to produce engineered tissues that better mimic the zonal collagenous structure of articular cartilage.

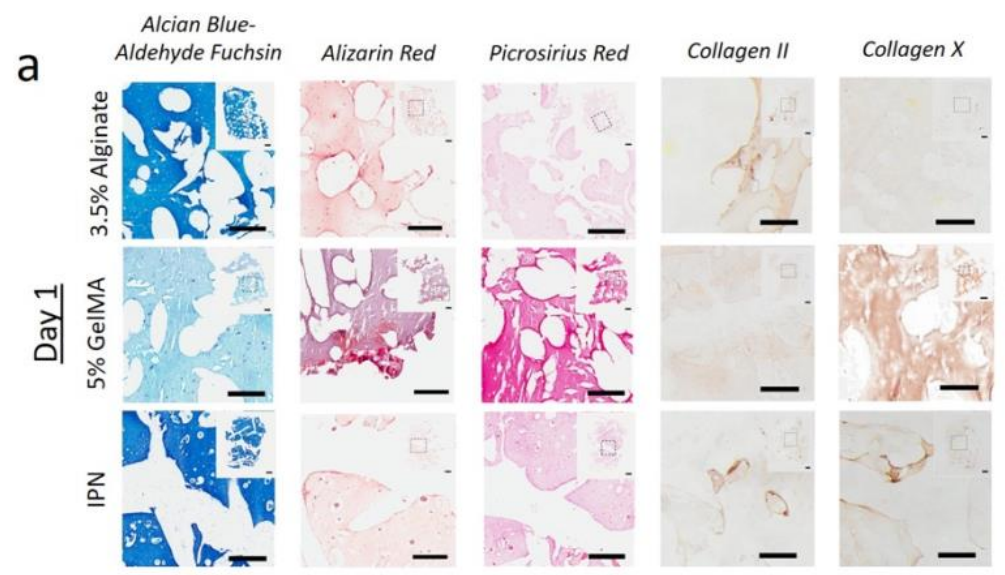

b

C
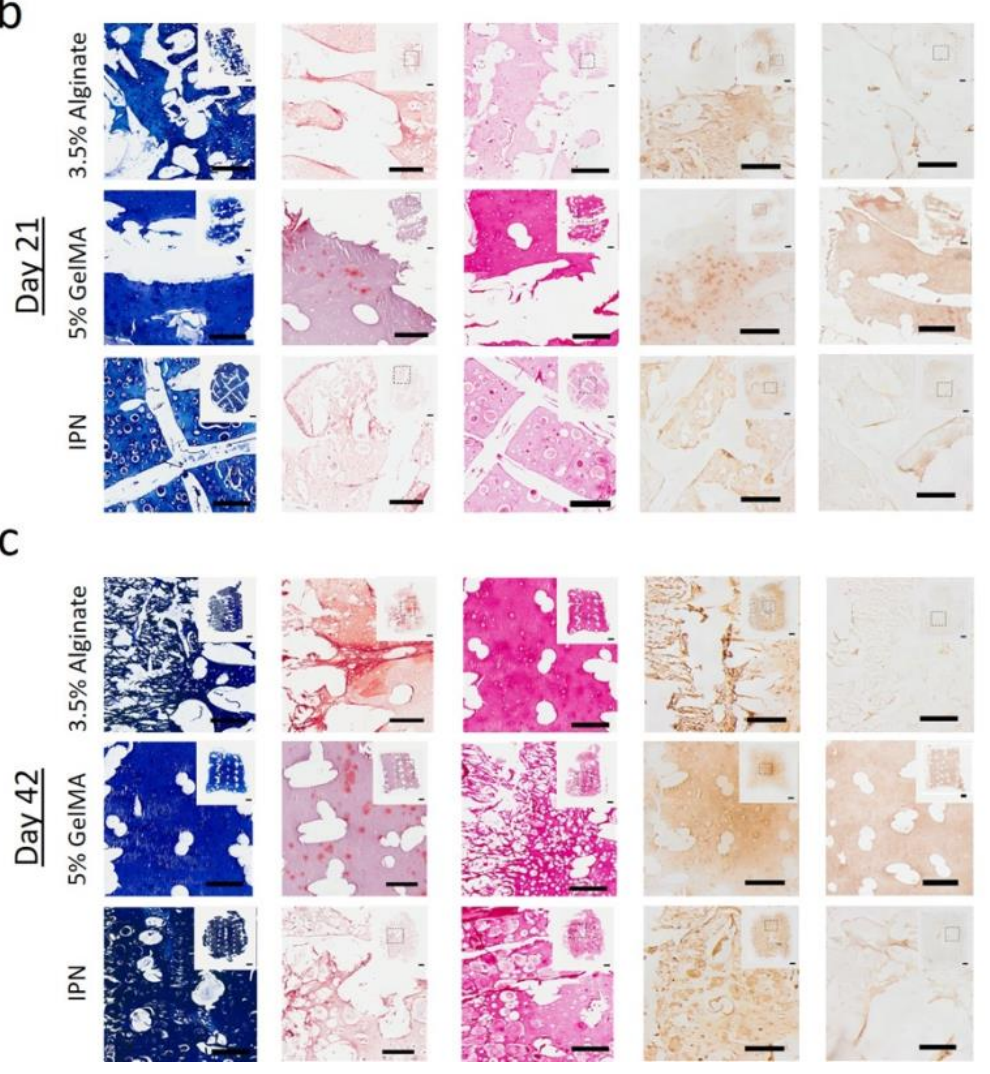

$3.5 \%$ Alginate

5\% GelMA

$\triangle$ IPN

d

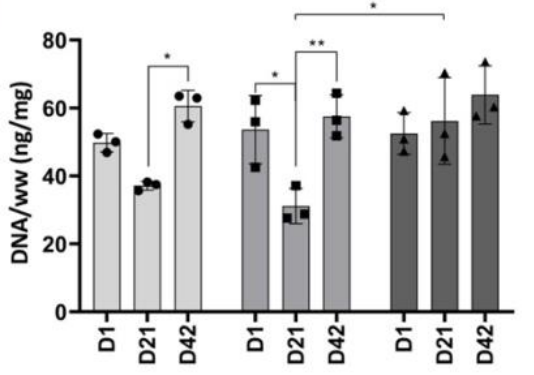

e

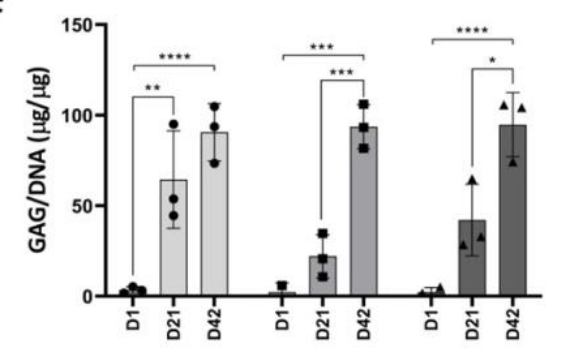

f

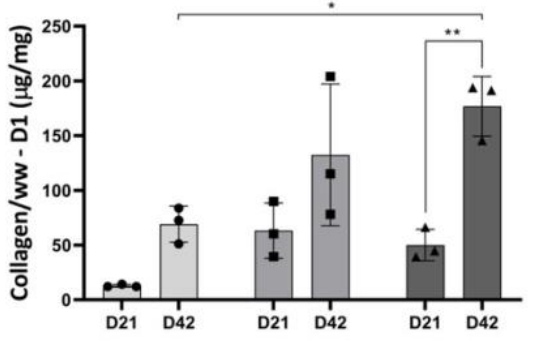

g

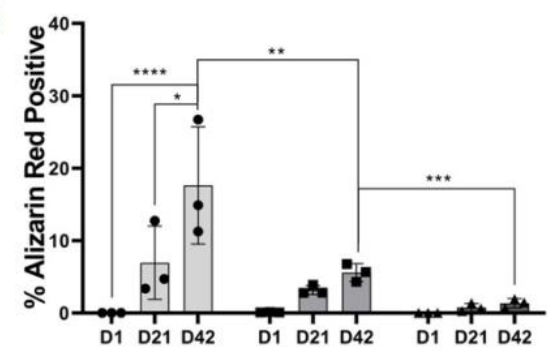


Figure 7. (a-c) Histological and immunohistochemical staining of all 3D bioprinted constructs groups after (a) 1, (b) 21 and (c) 42 days of in vitro culture. Scale bar: $500 \mu \mathrm{m}$. (d-f) Biochemical analysis of all composites, including (d) DNA content normalized to wet weight (ng/mg), (e) GAG/DNA and (f) collagen/DNA after day 1, 21 and 42 of in vitro culture. (g) Quantification of percentage of Alizarin Red staining within all three composite groups at day 1,21 and $42 .{ }^{*} p<0.05,{ }^{* *} p<0.01$, $* * * p<0.001$ and $* * * * p<0.0001 ; n=3$ per group.

\section{Conclusions}

This work represents a significant step towards developing biomechanically functional biomaterials for cartilage tissue engineering. The combination of alginate - GelMA IPN hydrogels with appropriately designed 3D printed PCL networks enables the engineering of composites with mechanical properties that are mimetic of normal articular cartilage. Importantly, such composite constructs provide encapsulated cells with an environment conducive to chondrogenesis, resulting in robust production of articular cartilage-like matrix.

\section{Acknowledgements}

This work was supported by a European Research Council Consolidator grant (647004) and a research grant from Science Foundation Ireland under grant number 12/IA/1554.

\section{References}

[1] Mow, V. C., S. C. Kuei, W. M. Lai and C. G. Armstrong 1980. Biphasic creep and stress relaxation of articular cartilage in compression? Theory and experiments. J Biomech Eng 102 73-84.

[2] Moutos, F. T., L. E. Freed and F. Guilak 2007. A biomimetic three-dimensional woven composite scaffold for functional tissue engineering of cartilage Nat Mater 6 162167.

[3] Gannon, A. R., T. Nagel and D. J. Kelly 2012. The role of the superficial region in determining the dynamic properties of articular cartilage Osteoarthritis Cartilage 20 1417-1425.

[4] Soltz, M. A. and G. A. Ateshian 2000. Interstitial Fluid Pressurization during Confined Compression Cyclical Loading of Articular Cartilage Ann Biomed Eng 28 150-159. [5] Guilak, F., D. L. Butler and S. A. Goldstein 2001. Functional tissue engineering: the role of biomechanics in articular cartilage repair. Clin Orthop Relat Res S295-305.

[6] Horkay, F. 2012. Interactions of cartilage extracellular matrix macromolecules Journal of Polymer Science, Part B: Polymer Physics 50 1699-1705.

[7] Kempson, G. E., H. Muir, S. A. V. Swanson and M. A. R. Freeman 1970. Correlations between stiffness and the chemical constituents of cartilage on the human femoral head BBA - General Subjects 215 70-77.

[8] Buckwalter, J. A., P. J. Roughley and L. C. Rosenberg 1994. Age-Related changes in cartilage proteoglycans: Quantitative electron microscopic studies Microsc Res Tech 28 398-408.
[9] Pastrama, M.-I., A. C. Ortiz, L. Zevenbergen, N. Famaey, W. Gsell, C. P. Neu, U. Himmelreich and I. Jonkers 2019. Combined enzymatic degradation of proteoglycans and collagen significantly alters intratissue strains in articular cartilage during cyclic compression $J$ Mech Behav Biomed Mater 98 383-394.

[10] Schmidt, M. B., V. C. Mow, L. E. Chun and D. R. Eyre 1990. Effects of proteoglycan extraction on the tensile behavior of articular cartilage J Orthop Res 8 353-363.

[11] Lu, X. L. and V. C. Mow 2008. Biomechanics of articular cartilage and determination of material properties Med Sci Sports Exerc 40 193-199.

[12] Bae, W. C., V. W. Wong, J. Hwang, J. M. Antonacci, G. E. Nugent-Derfus, M. E. Blewis, M. M. Temple-Wong and R. L. Sah 2008. Wear-lines and split-lines of human patellar cartilage: relation to tensile biomechanical properties. Osteoarthritis Cartilage 16 841-845.

[13] Kempson, G. E., M. A. Freeman and S. A. Swanson 1968. Tensile properties of articular cartilage. Nature 2201127 1128.

[14] Williamson, A. K., A. C. Chen, K. Masuda, E. J. M. A. Thonar and R. L. Sah 2003. Tensile mechanical properties of bovine articular cartilage: Variations with growth and relationships to collagen network components J Orthop Res 21 872-880.

[15] Park, S., C. T. Hung and G. A. Ateshian 2004. Mechanical response of bovine articular cartilage under dynamic unconfined compression loading at physiological stress levels Osteoarthritis Cartilage 12 65-73.

[16] Park, S., R. Krishnan, S. B. Nicoll and G. A. Ateshian 2003. Cartilage interstitial fluid load support in unconfined compression J Biomech 36 1785-1796.

[17] Federico, S. and W. Herzog 2008. On the anisotropy and inhomogeneity of permeability in articular cartilage Biomech Model Mechanobiol 7 367-378.

[18] Canal Guterl, C., C. T. Hung and G. A. Ateshian 2010. Electrostatic and non-electrostatic contributions of proteoglycans to the compressive equilibrium modulus of bovine articular cartilage $J$ Biomech 43 1343-1350.

[19] Bas, O., E. M. De-Juan-Pardo, C. Meinert, D. D'Angella, J. G. Baldwin, L. J. Bray, R. M. Wellard, S. Kollmannsberger, E. Rank, C. Werner, T. J. Klein, I. Catelas and D. W. Hutmacher 2017. Biofabricated soft network composites for cartilage tissue engineering Biofabrication 9025014.

[20] Almeida, H. V., G. M. Cunniffe, T. Vinardell, C. T. Buckley, F. J. O'Brien and D. J. Kelly 2015. Coupling Freshly Isolated CD44(+) Infrapatellar Fat Pad-Derived Stromal Cells with a TGF-beta3 Eluting Cartilage ECM-Derived Scaffold as 
a Single-Stage Strategy for Promoting Chondrogenesis $A d v$ Healthc Mater 4 1043-1053.

[21] Thorpe, S. D., T. Nagel, S. F. Carroll and D. J. Kelly 2013. Modulating gradients in regulatory signals within mesenchymal stem cell seeded hydrogels: a novel strategy to engineer zonal articular cartilage PLoS One 8 e60764.

[22] Cunniffe, G. M., P. J. Díaz-Payno, E. J. Sheehy, S. E. Critchley, H. V. Almeida, P. Pitacco, S. F. Carroll, O. R. Mahon, A. Dunne, T. J. Levingstone, C. J. Moran, R. T. Brady, F. J. O'Brien, P. A. J. Brama and D. J. Kelly 2019. Tissue-specific extracellular matrix scaffolds for the regeneration of spatially complex musculoskeletal tissues Biomaterials 188 63-73.

[23] Yang, J., Y. S. Zhang, K. Yue and A. Khademhosseini 2017. Cell-laden hydrogels for osteochondral and cartilage tissue engineering Acta Biomater 57 1-25.

[24] Bhattacharjee, M., J. Coburn, M. Centola, S. Murab, A. Barbero, D. L. Kaplan, I. Martin and S. Ghosh 2015. Tissue engineering strategies to study cartilage development, degeneration and regeneration Adv Drug Del Rev 84 107-122. [25] Kwon, H., W. E. Brown, C. A. Lee, D. Wang, N. Paschos, J. C. Hu and K. A. Athanasiou 2019. Surgical and tissue engineering strategies for articular cartilage and meniscus repair Nature Reviews Rheumatology.

[26] Izadifar, Z., X. Chen and W. Kulyk 2012. Strategic design and fabrication of engineered scaffolds for articular cartilage repair. Journal of functional biomaterials 3 799-838.

[27] Johnstone, B., M. Alini, M. Cucchiarini, G. Dodge, D. Eglin, F. Guilak, H. Madry, A. Mata, R. Mauck, C. Semino and M. Stoddart 2013. Tissue engineering for articular cartilage repair - the state of the art Eur Cells Mater 25 248267.

[28] Yang, X., Z. Lu, H. Wu, W. Li, L. Zheng and J. Zhao 2018. Collagen-alginate as bioink for three-dimensional (3D) cell printing based cartilage tissue engineering Materials Science and Engineering: C 83 195-201.

[29] Filardo, G., E. Kon, A. Roffi, A. Di Martino and M. Marcacci 2013. Scaffold-based repair for cartilage healing: A systematic review and technical note Arthroscopy - Journal of Arthroscopic and Related Surgery 29 174-186.

[30] Neumann, A. J., T. Quinn and S. J. Bryant 2016. Nondestructive evaluation of a new hydrolytically degradable and photo-clickable PEG hydrogel for cartilage tissue engineering Acta Biomater 39 1-11.

[31] Liao, I. C., F. T. Moutos, B. T. Estes, X. Zhao and F. Guilak 2013. Composite three-dimensional woven scaffolds with interpenetrating network hydrogels to create functional synthetic articular cartilage Adv Funct Mater 23 5833-5839. [32] Xu, C., G. Dai and Y. Hong 2019. Recent advances in high-strength and elastic hydrogels for 3D printing in biomedical applications Acta Biomater 95 50-59.

[33] Jeon, O., J.-Y. Shin, R. Marks, M. Hopkins, T.-H. Kim, H.-H. Park and E. Alsberg 2017. Highly Elastic and Tough Interpenetrating Polymer Network-Structured Hybrid Hydrogels for Cyclic Mechanical Loading-Enhanced Tissue Engineering Chem Mater 29 8425-8432.

[34] Brigham, M. D., M. Eng, A. Bick, E. Lo, A. Bendali, J. A. Burdick, D. Ph and A. Khademhosseini 2009.
Mechanically Robust and Bioadhesive Collagen SemiInterpenetrating Networks Tissue Engineering: Part A 15 1645-1653.

[35] Chimene, D., K. K. Lennox, R. R. Kaunas and A. K. Gaharwar 2016. Advanced Bioinks for 3D Printing: A Materials Science Perspective Ann Biomed Eng 44 20902102.

[36] Malda, J., J. Visser, F. P. Melchels, T. Jüngst, W. E. Hennink, W. J. A. Dhert, J. Groll and D. W. Hutmacher 2013. 25th anniversary article: Engineering hydrogels for biofabrication Adv Mater 25 5011-5028.

[37] Bian, L., C. Hou, E. Tous, R. Rai, R. L. Mauck and J. A. Burdick 2013. The influence of hyaluronic acid hydrogel crosslinking density and macromolecular diffusivity on human MSC chondrogenesis and hypertrophy Biomaterials 34 413-421.

[38] Sun, A. X., H. Lin, M. R. Fritch, H. Shen, P. G. Alexander, M. DeHart and R. S. Tuan 2017. Chondrogenesis of human bone marrow mesenchymal stem cells in 3dimensional, photocrosslinked hydrogel constructs: Effect of cell seeding density and material stiffness. Acta Biomater $\mathbf{5 8}$ 302-311.

[39] Visser, J., F. P. W. Melchels, J. E. Jeon, E. M. van Bussel, L. S. Kimpton, H. M. Byrne, W. J. a. Dhert, P. D. Dalton, D. W. Hutmacher and J. Malda 2015. Reinforcement of hydrogels using three-dimensionally printed microfibres. Nature communications 66933.

[40] Liao, I.-C., F. T. Moutos, B. T. Estes, X. Zhao and F. Guilak 2013. Composite Three-Dimensional Woven Scaffolds with Interpenetrating Network Hydrogels to Create Functional Synthetic Articular Cartilage Adv Funct Mater 23 5833-5839.

[41] Moffat, K. L., K. Goon, F. T. Moutos, B. T. Estes, S. J. Oswald, X. Zhao and F. Guilak 2018. Composite Cellularized Structures Created from an Interpenetrating Polymer Network Hydrogel Reinforced by a 3D Woven Scaffold Macromol Biosci 18001401800140.

[42] Murphy, S. V. and A. Atala 2014. 3D bioprinting of tissues and organs Nat Biotechnol 32 773-785.

[43] Daly, A. C., F. E. Freeman, T. Gonzalez-Fernandez, S. E. Critchley, J. Nulty and D. J. Kelly 2017. 3D Bioprinting for Cartilage and Osteochondral Tissue Engineering Adv Healthc Mater 6.

[44] Middleton, J. C. and A. J. Tipton 2000. Synthetic biodegradable polymers as orthopedic devices Biomaterials 21 2335-2346.

[45] Woodruff, M. A. and D. W. Hutmacher 2010. The return of a forgotten polymer - Polycaprolactone in the 21 st century Progress in Polymer Science (Oxford) 35 1217-1256.

[46] Olubamiji, A. D., Z. Izadifar, J. L. Si, D. M. L. Cooper, B. F. Eames and D. X. B. Chen 2016. Modulating mechanical behaviour of 3D-printed cartilage-mimetic PCL scaffolds: influence of molecular weight and pore geometry. Biofabrication 8025020.

[47] Shasteen, C. and Y. B. Choy 2011. Controlling degradation rate of poly(lactic acid) for its biomedical applications Biomedical Engineering Letters 1 163-167. 
[48] Pan, Z. and J. Ding 2012. Poly(lactide-co-glycolide) porous scaffolds for tissue engineering and regenerative medicine Interface Focus 2 366-377.

[49] Nair, L. S. and C. T. Laurencin 2007. Biodegradable polymers as biomaterials Progress in Polymer Science (Oxford) 32 762-798.

[50] Dawson, E., G. Mapili, K. Erickson, S. Taqvi and K. Roy 2008. Biomaterials for stem cell differentiation Adv Drug Del Rev $60215-228$.

[51] van Sliedregt, A., M. Knook, S. C. Hesseling, H. K. Koerten, K. de Groot and C. A. van Blitterswijk 1992. Cellular reaction on the intraperitoneal injection of four types of polylactide particulates Biomaterials 13 819-824.

[52] Hung, C. T., R. L. Mauck, C. C. B. Wang, E. G. Lima and G. A. Ateshian 2004. A paradigm for functional tissue engineering of articular cartilage via applied physiologic deformational loading Ann Biomed Eng 32 35-49.

[53] Fedorovich, N. E., J. Alblas, J. R. de Wijn, W. E. Hennink, A. J. Verbout and W. J. A. Dhert 2007. Hydrogels as Extracellular Matrices for Skeletal Tissue Engineering: State-of-the-Art and Novel Application in Organ Printing Tissue Eng 13 1905-1925.

[54] Augst, A. D., H. J. Kong and D. J. Mooney 2006. Alginate hydrogels as biomaterials Macromol Biosci 6 623633.

[55] Gasperini, L., J. F. Mano and R. L. Reis 2014. Natural polymers for the microencapsulation of cells Journal of the Royal Society Interface 11.

[56] Alsberg, E., H. J. Kong, Y. Hirano, M. K. Smith, A. Albeiruti and D. J. Mooney 2003. Regulating bone formation via controlled scaffold degradation J Dent Res 82 903-908. [57] Lee, K. Y. and D. J. Mooney 2012. Alginate: Properties and biomedical applications Progress in Polymer Science (Oxford) 37 106-126.

[58] Jeon, O., C. Powell, S. M. Ahmed and E. Alsberg 2010. Biodegradable, photocrosslinked alginate hydrogels with independently tailorable physical properties and cell adhesivity Tissue Engineering - Part A 16 2915-2925.

[59] Jeon, O., J. Y. Shin, R. Marks, M. Hopkins, T. H. Kim, H. H. Park and E. Alsberg 2017. Highly elastic and tough interpenetrating polymer network-structured hybrid hydrogels for cyclic mechanical loading-enhanced tissue engineering Chem Mater 29 8425-8432.

[60] Sun, J. Y., X. Zhao, W. R. K. Illeperuma, O. Chaudhuri, K. H. Oh, D. J. Mooney, J. J. Vlassak and Z. Suo 2012. Highly stretchable and tough hydrogels Nature 489 133-136.

[61] Zhu, M., Y. Wang, G. Ferracci, J. Zheng, N. J. Cho and B. H. Lee 2019. Gelatin methacryloyl and its hydrogels with an exceptional degree of controllability and batch-to-batch consistency Sci Rep 9 1-13.

[62] Wang, H., L. Zhou, J. Liao, Y. Tan, K. Ouyang, C. Ning, G. Ni and G. Tan 2014. Cell-laden photocrosslinked GelMADexMA copolymer hydrogels with tunable mechanical properties for tissue engineering J Mater Sci Mater Med 25 2173-2183.

[63] Van Den Bulcke, A. I., B. Bogdanov, N. De Rooze, E. H. Schacht, M. Cornelissen and H. Berghmans 2000. Structural and Rheological Properties of Methacrylamide Modified Gelatin Hydrogels Biomacromolecules 1 31-38.

[64] Mesallati, T., E. J. Sheehy, T. Vinardell, C. T. Buckley and D. J. Kelly 2015. Tissue engineering scaled-up, anatomically shaped osteochondral constructs for joint resurfacing Eur Cells Mater 30 163-186.

[65] Schipani, R., D. R. Nolan, C. Lally, D. J. Kelly, R. Schipani, D. R. Nolan, C. Lally and D. J. Kelly 2019. Integrating finite element modelling and $3 \mathrm{D}$ printing to engineer biomimetic polymeric scaffolds for tissue engineering 8207.

[66] Castilho, M., G. Hochleitner, W. Wilson, B. van Rietbergen, P. D. Dalton, J. Groll, J. Malda and K. Ito 2018. Mechanical behavior of a soft hydrogel reinforced with threedimensional printed microfibre scaffolds Sci Rep 81245.

[67] Mow, V. C. and X. E. Guo 2002. MechanoElectrochemical Properties Of Articular Cartilage: Their Inhomogeneities and Anisotropies Annu Rev Biomed Eng 4 175-209.

[68] Gu, L., O. Chaudhuri, H.-p. Lee, D. J. Mooney and M. E. Levenston 2017. Mechanical confinement regulates cartilage matrix formation by chondrocytes Nature Materials 16 12431251.

[69] Chaudhuri, O., L. Gu, D. Klumpers, M. Darnell, S. A. Bencherif, J. C. Weaver, N. Huebsch, H. P. Lee, E. Lippens, G. N. Duda and D. J. Mooney 2016. Hydrogels with tunable stress relaxation regulate stem cell fate and activity Nat Mater 15 326-334.

[70] Metzger, T. A., S. A. Schwaner, A. J. LaNeve, T. C. Kreipke and G. L. Niebur 2015. Pressure and shear stress in trabecular bone marrow during whole bone loading $J$ Biomech 48 3035-3043.

[71] Zhao, X., N. Huebsch, D. J. Mooney and Z. Suo 2010. Stress-relaxation behavior in gels with ionic and covalent crosslinks J Appl Phys 10763509.

[72] Beck, E. C., M. Barragan, M. H. Tadros, S. H. Gehrke and M. S. Detamore 2016. Approaching the compressive modulus of articular cartilage with a decellularized cartilagebased hydrogel Acta Biomater 38 94-105.

[73] Little, C. J., N. K. Bawolin and X. Chen 2011. Mechanical Properties of Natural Cartilage and TissueEngineered Constructs Tissue Engineering Part B: Reviews 17 213-227.

[74] Jurvelin, J. S., M. D. Buschmann and E. B. Hunziker 1997. Optical and mechanical determination of Poisson's ratio of adult bovine humeral articular cartilage J Biomech 30235 241.

[75] Erickson, I. E., A. H. Huang, C. Chung, R. T. Li, J. A. Burdick and R. L. Mauck 2009. Differential Maturation and Structure-Function Relationships in Mesenchymal Stem Celland Chondrocyte-Seeded Hydrogels Tissue Engineering Part A 15 1041-1052.

[76] Hofmann, S., S. Knecht, R. Langer, D. L. Kaplan, G. Vunjak-Novakovic, H. P. Merkle and L. Meinel 2006. Cartilage-like Tissue Engineering Using Silk Scaffolds and Mesenchymal Stem Cells Tissue Eng 0060915113954001. 
[77] Critchley, S. E. and D. J. Kelly 2017. Bioinks for bioprinting functional meniscus and articular cartilage Journal of 3D Printing in Medicine 1 269-290.

[78] Daly, A. C., S. E. Critchley, E. M. Rencsok and D. J. Kelly 2016. A comparison of different bioinks for 3D bioprinting of fi brocartilage and hyaline cartilage Biofabrication 8 1-10.

[79] Critchley, S., G. Cunniffe, A. O'Reilly, P. Diaz-Payno, R. Schipani, A. McAlinden, D. Withers, J. Shin, E. Alsberg and D. J. Kelly 2019. Regeneration of Osteochondral Defects Using Developmentally Inspired Cartilaginous Templates Tissue Eng Part A 25 159-171.

[80] Beck, E. C., M. Barragan, M. H. Tadros, S. H. Gehrke and M. S. Detamore 2016. Approaching the compressive modulus of articular cartilage with a decellularized cartilagebased hydrogel. Acta Biomater 38 94-105.

[81] Korhonen, R. K. and J. S. Jurvelin 2010. Compressive and tensile properties of articular cartilage in axial loading are modulated differently by osmotic environment Med Eng Phys 32 155-160

[82] Gannon, A. R., T. Nagel, A. P. Bell, N. C. Avery and D. J. Kelly 2015. Postnatal changes to the mechanical properties of articular cartilage are driven by the evolution of its Collagen network European Cells and Materials 29 105-123.

[83] Gao, L. L., C. Q. Zhang, H. Gao, Z. D. Liu and P. P. Xiao 2014. Depth and rate dependent mechanical behaviors for articular cartilage: Experiments and theoretical predictions Mater Sci Eng, C 38 244-251.

[84] Athanasiou, K. A., A. Agarwal and F. J. Dzida 1994. Comparative study of the intrinsic mechanical properties of the human acetabular and femoral head cartilage J Orthop Res 12 340-349.

[85] Kinnunen, J., S. Saarakkala, M. Hauta-Kasari, P. Vahimaa and J. S. Jurvelin 2011. Optical spectral reflectance of human articular cartilage - relationships with tissue structure, composition and mechanical properties Biomedical Optics Express 21394.

[86] Bian, L., E. G. Lima, S. L. Angione, K. W. Ng, D. Y. Williams, D. Xu, A. M. Stoker, J. L. Cook, G. A. Ateshian and C. T. Hung 2008. Mechanical and biochemical characterization of cartilage explants in serum-free culture. $J$ Biomech 41 1153-1159.

[87] Levett, P. A., F. P. W. Melchels, K. Schrobback, D. W. Hutmacher, J. Malda and T. J. Klein 2014. A biomimetic extracellular matrix for cartilage tissue engineering centered on photocurable gelatin, hyaluronic acid and chondroitin sulfate Acta Biomater 10 214-223.

[88] Levett, P. A., F. P. W. Melchels, K. Schrobback, D. W. Hutmacher, J. Malda and T. J. Klein 2014. Chondrocyte redifferentiation and construct mechanical property development in single-component photocrosslinkable hydrogels Journal of Biomedical Materials Research - Part A 102 2544-2553.

[89] Schuurman, W., P. a. Levett, M. W. Pot, P. R. van Weeren, W. J. a. Dhert, D. W. Hutmacher, F. P. W. Melchels, T. J. Klein and J. Malda 2013. Gelatin-methacrylamide hydrogels as potential biomaterials for fabrication of tissueengineered cartilage constructs. Macromol Biosci 13 551-561.
[90] Awad, H. A., M. Q. Wickham, H. A. Leddy, J. M. Gimble and F. Guilak 2004. Chondrogenic differentiation of adiposederived adult stem cells in agarose, alginate, and gelatin scaffolds Biomaterials 25 3211-3222.

[91] Rowley, J. A., G. Madlambayan and D. J. Mooney 1999. Alginate hydrogels as synthetic extracellular matrix materials Biomaterials 20 45-53.

[92] Benya, P. D. and J. D. Shaffer 1982. Dedifferentiated chondrocytes reexpress the differentiated collagen phenotype when cultured in agarose gels Cell 30 215-224.

[93] Fischer, J., A. Dickhut, M. Rickert and W. Richter 2010. Human articular chondrocytes secrete parathyroid hormonerelated protein and inhibit hypertrophy of mesenchymal stem cells in coculture during chondrogenesis Arthritis Rheum 62 2696-2706.

[94] Bian, L., D. Y. Zhai, R. L. Mauck and J. A. Burdick 2011. Coculture of human mesenchymal stem cells and articular chondrocytes reduces hypertrophy and enhances functional properties of engineered cartilage Tissue Engineering - Part A 17 1137-1145.

[95] Kang, N., X. Liu, Y. Guan, J. Wang, F. Gong, X. Yang, L. Yan, Q. Wang, X. Fu, Y. Cao and R. Xiao 2012. Effects of co-culturing BMSCs and auricular chondrocytes on the elastic modulus and hypertrophy of tissue engineered cartilage Biomaterials 33 4535-4544.

[96] Cooke, M. E., A. A. Allon, T. Cheng, A. C. Kuo, H. T. Kim, T. P. Vail, R. S. Marcucio, R. A. Schneider, J. C. Lotz and T. Alliston 2011. Structured three-dimensional co-culture of mesenchymal stem cells with chondrocytes promotes chondrogenic differentiation without hypertrophy. Osteoarthritis Cartilage 19 1210-1218.

[97] Engler, A. J., S. Sen, H. L. Sweeney and D. E. Discher 2006. Matrix Elasticity Directs Stem Cell Lineage Specification Cell 126 677-689.

[98] Nguyen, Q. T., Y. Hwang, A. C. Chen, S. Varghese and R. L. Sah 2012. Cartilage-like mechanical properties of poly (ethylene glycol)-diacrylate hydrogels Biomaterials 33 66826690.

[99] Zhu, D., X. Tong, P. Trinh and F. Yang 2018. Mimicking Cartilage Tissue Zonal Organization by Engineering TissueScale Gradient Hydrogels as 3D Cell Niche Tissue Engineering - Part A 24 1-10. 


\title{
Reinforcing interpenetrating network hydrogels with 3D printed polymer networks to engineer cartilage mimetic composites
}

\author{
Rossana Schipani $^{1,3}$, Stefan Scheurer ${ }^{1,2}$, Romain Florentin ${ }^{1,3}$, Susan E Critchley ${ }^{1,3}$ and Daniel J Kelly Re, $^{1,3,5}$ \\ 1 Trinity Centre for Biomedical Engineering, Trinity Biomedical Sciences Institute, Trinity College Dublin, Dublin, Ireland \\ 2 Orthopaedics and Sports Medicine, Trinity College Dublin, Dublin, Ireland \\ 3 Department of Mechanical and Manufacturing Engineering, School of Engineering, Trinity College Dublin, Ireland \\ 4 Department of Anatomy, Royal College of Surgeons in Ireland, Dublin, Ireland \\ 5 Advanced Materials and Bioengineering Research Centre (AMBER), Royal College of Surgeons in Ireland and Trinity College Dublin, \\ Dublin, Ireland \\ E-mail: kellyd9@tcd.ie
}

Received xxxxxx

Accepted for publication $\mathrm{xxxxxx}$

Published xxxxxx

\begin{abstract}
Engineering constructs that mimic the complex structure, composition and biomechanics of the articular cartilage represents a promising route to joint regeneration. Such tissue engineering strategies require the development of biomaterials that mimic the mechanical properties of articular cartilage whilst simultaneously providing an environment supportive of chondrogenesis. Here three-dimensional (3D) bioprinting is used to develop polycaprolactone (PCL) fibre networks to mechanically reinforce interpenetrating network (IPN) hydrogels consisting of alginate and gelatin methacryloyl (GelMA). Inspired by the significant tension-compression nonlinearity of the collagen network in articular cartilage, we printed reinforcing PCL networks with different ratios of tensile to compressive modulus. Synergistic increases in compressive modulus were observed when IPN hydrogels were reinforced with PCL networks that were relatively soft in compression and stiff in tension. The resulting composites possessed equilibrium and dynamic mechanical properties that matched or approached that of native articular cartilage. Finite Element (FE) modelling revealed that the reinforcement of IPN hydrogels with specific PCL networks limited radial expansion and increased the hydrostatic pressure generated within the IPN upon the application of compressive loading. Next, multiple-tool biofabrication techniques were used to 3D bioprint PCL reinforced IPN hydrogels laden with a co-culture of bone marrow-derived stromal cells (BMSCs) and chondrocytes (CCs). The bioprinted biomimetic composites were found to support robust chondrogenesis, with encapsulated cells producing hyaline-like cartilage that stained strongly for sGAG and type II collagen deposition, and negatively for type X collagen and calcium deposition. Taken together, these results demonstrate how 3D bioprinting can be used to engineer constructs that are both pro-chondrogenic and biomimetic of the mechanical properties of articular cartilage.
\end{abstract}

Keywords: articular cartilage, tissue engineering, interpenetrating network hydrogels, polycaprolactone, 3D bioprinting, finite element modelling, biomimetic scaffolds

\section{Introduction}

Articular cartilage is a thin layer of connective tissue that lines the ends of bones within synovial joints. Under normal physiological conditions, cartilage provides a smooth, lubricated surface for articulation that facilitates load support and distribution across the joint. Biomechanically it functions as a multiphasic, fibre-reinforced material that has anisotropic, nonlinear and viscoelastic properties [1-3]. The complex load bearing properties of articular cartilage strongly depend on the anisotropic nature of the tissue, consisting primarily of collagen and proteoglycans, and the associated tensioncompression nonlinearity $[1,2,4,5]$. The negatively charged 
proteoglycans within articular cartilage generate an osmotic swelling pressure, which is balanced by a pre-stress that is developed in the collagen network $[3,6]$. The compressive properties of cartilage typically increase as the proteoglycans content increases, and the loss of proteoglycans has been shown to make the tissue more prone to microdamage during mechanical loading [1, 7-10]. The architecture and organization of the collagen fibres determine the intrinsic tensile stiffness and strength of cartilage [10-12]. The tensile modulus of articular cartilage is significantly higher than the compressive modulus $[13,14]$. When cartilage is tested in unconfined compression, this tension-compression nonlinearity enhances fluid pressurization and elevates the dynamic modulus of the tissue $[15,16]$, as the collagen network restricts lateral expansion of the tissue during compressive loading. The relative importance of the different matrix components on the overall mechanical properties of articular cartilage has been studied by depleting cartilage samples of collagen and/or proteoglycans [2, 9, 10, 17]. For example, the compressive modulus of the proteoglycandepleted cartilage matrix may be as low as $3 \mathrm{kPa}$, representing less than $2 \%$ of the normal tissue modulus [18]. Therefore, the collagen in articular cartilage can be considered as a fibrillar matrix that primarily sustains tension. Biomaterial strategies that aim to mimic articular cartilage should consider such complexities in their design.

As articular cartilage has poor regenerative capacity, damage to this tissue impairs its mechanical function and can lead to an abnormal loading within the joint. If left untreated, the damage can progress to osteoarthritis of the joint, causing significant pain and disability. This motivates the need for new regenerative approaches capable of restoring the normal biomechanics of the joint.

A number of tissue engineered strategies to engineer cartilage replacements have been developed [19-27]. A typical approach is to design biomaterial environments capable of supporting chondrogenesis; this commonly involves combining cells and/or bioactive molecules with hydrogels as a scaffolding material due to their high water content and attractive transport properties [28-31]. Other than presenting pro-chondrogenic biological cues to the encapsulated cells, such biomaterial scaffolds must also provide a suitable mechanical environment capable of instructing cell fate as well as withstanding the challenging physiological loading conditions experienced in vivo upon implantation into the body [27]. Commonly used single network hydrogels (e.g. alginate, GelMA, agarose, polyethylene glycol, etc.) have been extensively studied and have been shown to provide an environment that is conducive to chondrogenesis. However, they are typically mechanically weak and unable to withstand the challenging environment of the joint. Recently, more complex hydrogel systems such as IPN hydrogels, characterized by the combination of multiple polymer networks have been investigated. Within such IPN hydrogels individual polymer networks are physically entangled with each other and crosslinked only with themselves using specific chemistries [32-35]. In general, such IPN hydrogels have displayed synergistic increases in mechanical properties, including increased toughness and elasticity when compared to the single hydrogel components. However, they do not mimic many of the features of load bearing tissues like articular cartilage, such as anisotropy and tensioncompression nonlinearity. Furthermore, engineering IPN hydrogels with bulk mechanical properties approaching that of articular cartilage typically requires very high hydrogel polymer concentrations, resulting in biomaterial environments that are not compatible with robust extracellular matrix (ECM) deposition by encapsulated cells [36-38].

To address these concerns, hydrogels can be reinforced with networks of fibres to produce composites with improved mechanical properties [2, 31, 39-41]. However, these techniques typically involve the use of complex biofabrication strategies (e.g. weaving, melt electrowriting) that are challenging to scale and personalize to specific patient anatomies. In contrast, Fused Deposition Modelling (FDM) can be used to $3 \mathrm{D}$ print fibre networks that are relatively simple to scale, with internal and external architectures that are easily defined based on medical imaging $[42,43]$. This is achieved using PCL, a synthetic thermoplastic polymer which has been extensively used in the field of 3D bioprinting [44]. Advantages include ease of shaping and manufacture due to its low meting temperature $\left(\sim 60^{\circ} \mathrm{C}\right)$, biocompatibility, elasticity and mechanical properties [45]. The mechanical properties of PCL scaffolds can also be tuned by modifying the molecular weight and the scaffold architecture [46]. Other synthetic polymers such as poly(lactic acid) (PLA) and poly(lactic-co-glycolic acid) (PLGA) are also biocompatible with FDM, with PLGA also allowing for more tailorable degradation rates [47-49]. A concern with the use of PLGA is the release of acidic products as the polymer degrades, which can induce inflammation $[50,51]$. Another major disadvantage is the higher melting temperature of PLA and PLGA compared to PCL, which is not ideal when co-printing with live cells.

In this study, alginate - GelMA IPNs were reinforced with PCL networks printed using FDM to develop biomechanically competent composite constructs for cartilage tissue engineering. [30, 52]. Alginate is a widely used biomaterial in tissue engineering because of its excellent biocompatibility, relatively high control over stiffness and capability to form highly porous structures which facilitate the diffusion of large molecules in and out of the hydrogel [53-55]. However, alginate is often limited under mechanical loading due to its brittleness and low elasticity [56-58]. GelMA is a gelatinbased material that has high biocompatibility, degrades in response to matrix metalloproteinases (MMP), possesses 
RGD sequences that favour cell adhesion and is highly elastic $[59,60]$. GelMA also possesses relatively poor mechanical strength and toughness [59, 61, 62], however IPNs of alginate and GelMA have previously been shown to have superior mechanical properties to their individual hydrogel components and to be supportive of cell growth and differentiation [59]. Here, alginate, GelMA and alginate GelMA IPN hydrogel scaffolds were first mechanically characterized to investigate the benefit of combining the two single hydrogel components for engineering cartilage-mimetic hydrogels. These hydrogel bioinks were then reinforced with 3D printed PCL networks with architectures and mechanical properties inspired by the collagen network of articular cartilage. The reinforcement mechanism resulting from the combination of PCL networks with IPN hydrogels was examined using Finite Element Analysis (FEA). Finally, the ability of 3D bioprinted cell-laden PCL reinforced IPN constructs to support chondrogenesis was assessed in vitro over 6 weeks of culture.

\section{Materials and methods}

\subsection{Materials preparation}

Alginate was prepared by dissolving Alginate (PRONOVA UP LVG) in DMEM to make up a final concentration of $3.5 \%$ w/v. Cylindrical constructs were obtained by pouring the alginate solution into $2 \mathrm{~mm}$ high- $6 \mathrm{~mm}$ diameter agarose$\mathrm{CaCl}_{2}$ moulds (DMEM, 3\% w/v agarose, $45 \mathrm{mM} \mathrm{CaCl} 2$ ) and allowed to crosslink for $20 \mathrm{~min}$ at room temperature. GelMA synthesis was previously described [63]. Briefly, GelMA was synthesized by reaction of porcine type A gelatin (SigmaAldrich, average molecular weight $40-50 \mathrm{kDa}$ ) with methacrylic anhydride (Sigma-Aldrich) at $50{ }^{\circ} \mathrm{C}$ for $4 \mathrm{~h}$. To achieve a high degree of functionalization, $10 \mathrm{~mL}$ of methacrylic anhydride was added to a $10 \% \mathrm{w} / \mathrm{v}$ gelatin solution in phosphate-buffered saline (PBS) under stirring for $1 \mathrm{~h}$. The functionalized polymer was dialyzed against distilled water for 7 days at $40{ }^{\circ} \mathrm{C}$ to remove unreacted methacrylic anhydride, freeze-dried and stored at $-20{ }^{\circ} \mathrm{C}$ protected from light until further use. GelMA constructs were formed by dissolving GelMA in DMEM (including $0.05 \% \mathrm{v} / \mathrm{v}$ Irgacure (Sigma Aldrich) as photoinitiator) obtaining final concentrations of 5, 10 and $15 \%$ w/v. The GelMA solution was poured into polydimethylsiloxane (PDMS) moulds (same dimensions as for the agarose- $\mathrm{CaCl}_{2}$ moulds) and exposed to UV light for $15 \mathrm{~min}\left(365 \mathrm{~nm}, 5 \mathrm{~mW} / \mathrm{cm}^{2}\right)$ to perform the crosslinking. Alginate - GelMA IPN hydrogels were prepared by combining alginate solution (DMEM, 7\% w/v) with GelMA solution of various concentrations (DMEM, 10, 20 and $30 \% \mathrm{w} / \mathrm{v}$ ) containing $0.05 \% \mathrm{w} / \mathrm{v}$ Irgacure in $1: 1$ ratio. The final mixture was poured into the same agarose- $\mathrm{CaCl}_{2}$ moulds used for alginate constructs and cured with $45 \mathrm{mM} \mathrm{CaCl}_{2}$ and under UV light exposure for 20 and 15 min respectively.

\subsection{Isolation and expansion of MSCs and CCS}

Bone marrow was removed from the femoral shaft of a porcine donor and washed in high-glucose Dulbecco's Modified Eagle Medium (hgDMEM) (Biosciences) supplemented with $10 \%$ foetal bovine serum (FBS), $1 \%$ penicillin (100 U/mL) - streptomycin $(100 \mu \mathrm{g} / \mathrm{mL})$ (all Bioscience) and amphotericin B $(2.5 \mu \mathrm{g} / \mathrm{mL})$ (SigmaAldrich). A homogenous cell suspension was achieved by triturating with a $18 \mathrm{G}$ needle. The solution was centrifuged twice at $650 \mathrm{~g}$ for $5 \mathrm{~min}$, with removal of the supernatant. The resultant cell pellet was triturated, and the cell suspension was filtered through a $40 \mu \mathrm{m}$ cell sieve (Sarstedt). Cell counting was performed with trypan blue and acetic acid before plating at a density of $5 \times 103$ cells $/ \mathrm{cm}_{2}$ and maintained in a humidified chamber. Following colony formation, cells were trypsinized, counted and re-plated for a further passage at a density of $5 \mathrm{x}$ 103 cells/cm2. FGF-2 (Prospec-Tany TechnoGene Ltd) was added to the media which was changed twice weekly.

Cartilage tissue from another porcine donor was rinsed with PBS, weighed and finely diced. Chondrocytes were isolated by digestion in hgDMEM containing $1 \%$ penicillin (100 $\mathrm{U} / \mathrm{mL})-$ streptomycin $(100 \mu \mathrm{g} / \mathrm{mL})$ and $8 \mathrm{~mL} / \mathrm{g}$ of collagenase type II $(350 \mathrm{U} / \mathrm{mL})$ for $12-14 \mathrm{~h}$ under constant rotation at 37 ${ }^{\circ} \mathrm{C}$. The resulting cell suspension was filtered through a $40 \mu \mathrm{m}$ cell sieve, centrifuged and rinsed with PBS twice. Cell number and viability were determined using a haemocytometer and $0.4 \%$ trypan blue staining.

All expansion was conducted in low oxygen conditions $\left(5 \% \mathrm{pO}_{2}\right)$ and media was changed twice weekly.

\subsection{D bioprinting process}

All 3D printed constructs were fabricated using the 3D Discovery bioplotter purchased from RegenHU with spatial resolution of $\pm 5 \mu \mathrm{m}$. Porous PCL (CAPA 6500D, Perstorp, Mn $50 \mathrm{kDa}$ ) scaffolds with two different geometries named Aligned and Double Offset were printed using a FDM printhead. All printing parameters are described in Table 1.

For the in vitro evaluation, previously printed PCL scaffolds were sterilized with ethylene oxide (EtO). Prior to hydrogels printing, the PCL constructs were placed in each well of a 6-well plate by means of 3D printed poly lactic acid inserts containing PDMS moulds that kept the scaffolds in place in the centre of the well. This allowed for $\mathrm{Z}$-direction printing of the hydrogel bioinks through microextrusion bioprinting. 
Table 1. Summary of FDM and microextrusion printing parameters for PCL and hydrogels.

\section{Printing Parameters $\quad$ FDM printing Microextrusion printing}

\begin{tabular}{|c|c|c|c|c|}
\hline Material & PCL & $3.5 \%$ alginate & $5 \%$ gelMA & IPN \\
\hline Needle (Gauge) & 30 & 23 & 23 & 23 \\
\hline Pressure (bar) & 1 & 0.5 & 0.3 & 1.3 \\
\hline Screw Speed (revs/m) & 10 & - & - & - \\
\hline Temperature $\left({ }^{\circ} \mathrm{C}\right)$ & $86($ tank), 78 (needle) & - & 13 & 13 \\
\hline Layer Thickness (mm) & 0.1 & - & - & - \\
\hline Extrusion Speed $(\mathrm{mm} / \mathrm{s})$ & 6 & 4 & 2 & 4 \\
\hline
\end{tabular}

To form the single hydrogel component bioinks, alginate and GelMA were dissolved at a concentration of 7 and $10 \%$ w/v respectively. To produce the IPN bioink, alginate and GelMA were firstly dissolved to 14 and $20 \%$ w/v respectively and then mixed together in 1:1 ratio using a luer lock system (7 and 10\% w/v alginate and GelMA final respectively). As co-cultures of BMSCs and CCs have been shown to support stable cartilage tissue formation resistant to hypertrophy and endochondral ossification in vivo [64], using the above mentioned luer lock system, the obtained bioinks were then combined with a mixture of BMSCs and CCs (3:1 ratio) in $1: 1$ ratio $(3.5 \% \mathrm{w} / \mathrm{v}$ alginate final, $5 \% \mathrm{w} / \mathrm{v}$ GelMA final, $3.5 \% \mathrm{w} / \mathrm{v}$ alginate $-5 \%$ w/v GelMA IPN final, 20 x 106 cells $/ \mathrm{mL}$ ). Cellencapsulated bioinks were loaded into a syringe and the plunger was moved up and down until air bubbles were collected at the tip of the syringe and could be removed. After minimizing the presence of air bubbles, individual hydrogel components were mixed using a dual syringe approach to form the IPN.

Using a z-direction bioprinting approach, the pores of the previously printed PCL networks were loaded with either alginate, GelMA or alginate-GelMA IPN bioinks. Z-direction bioprinting involved using a generated custom G-code to deposit the required bioink by moving the needle in the $\mathrm{z}$ direction through the open pores of the PCL networks. To make sure that cell-encapsulated bioinks were homogeneously distributed, the PCL networks were soaked in $45 \mathrm{mM} \mathrm{CaCl}_{2}$ and let rotate overnight before bioprinting. This initiated the crosslinking process of the bioinks as soon as they were extruded, avoiding settling of the hydrogels at the bottom of the PCL scaffolds as well as formation of empty spaces. See Table 2 for printing parameters. Following the fabrication process, the constructs were fully crosslinked as follows: PCL + alginate scaffolds were immersed in a bath of $45 \mathrm{mM} \mathrm{CaCl}_{2}$ for 20 mins; PCL + GelMA constructs underwent UV light exposure for 15 mins; PCL + IPN constructs were subjected to both $\mathrm{CaCl}_{2}(45 \mathrm{mM})$ and $\mathrm{UV}$ light crosslinking for 15 and 20 min respectively.

\subsection{In vitro culture conditions}

3D bioprinted scaffolds were cultured for 6 weeks in chondrogenically defined media consisting of hgDMEM supplemented with $1 \%$ penicillin $(100 \mathrm{U} / \mathrm{mL})$ - streptomycin $(100 \mu \mathrm{g} / \mathrm{mL})$, sodium pyruvate $(100 \mu \mathrm{g} / \mathrm{mL})$, L-proline (40 $\mu \mathrm{g} / \mathrm{mL})$, L-ascorbic acid 2-phosphate $(50 \mu \mathrm{g} / \mathrm{mL})$, linoleic acid $(4.7 \mu \mathrm{g} / \mathrm{mL})$, bovine serum albumin $(1.5 \mathrm{mg} / \mathrm{mL}), 1 \mathrm{x}$ insulin-transferrin-selenium, dexamethasone (100 nM) (all Sigma-Aldrich) and human TGF-B3 (10 ng/mL) (ProspecTany TechnoGene Ltd). Media change was performed twice weekly. To test whether a co-culture of MSCs and CCs within a bioink is capable of promoting the development of phenotypically stable articular cartilage, constructs were maintained at $5 \% \mathrm{pO} 2$ for the first 3 weeks of culture to induce robust chondrogenesis, and then switched to $20 \% \mathrm{pO} 2$ for the final 3 weeks to test the capacity of the co-culture to maintain a stable cartilage phenotype.

\subsection{Live/Dead confocal microscopy}

Cell viability was evaluated $24 \mathrm{~h}$ post-printing using a Live/Dead assay kit (Bioscience). Bioprinted cell-laden scaffolds were rinsed with phenol free medium and incubated in a solution containing $4 \mu \mathrm{M}$ ethidium homodimer- 1 and 2 $\mu \mathrm{M}$ calcein for $1 \mathrm{~h}$. Following incubation, the scaffolds were rinsed again and imaged with Olympus FV-1000 PointScanning Confocal Microscope t 488 and $543 \mathrm{~nm}$ channels. Cell viability was quantified using Image-J software. 


\subsection{Biochemical analysis}

The biochemical content of all constructs was quantified. Samples were digested with papain $(125 \mu \mathrm{g} / \mathrm{mL})$ in $0.1 \mathrm{M}$ sodium acetate, $5 \mathrm{mM}$ L-cysteine- $\mathrm{HCl}, 0.05 \mathrm{M}$ ethylenediamine-tetracetic acid (EDTA) (all Sigma-Aldrich) and $\mathrm{pH} 6$ under constant rotation at $60{ }^{\circ} \mathrm{C}$ for $18 \mathrm{~h}$. DNA content was assessed using the Hoechst Bisbenzimide 33258 dye assay. The amount of sulphated glycosaminoglycans (sGAG) was measured using the dimethyl methylene blue-binding assay (DMMB) (Blyscan, Bicolor Ltd.). Total collagen content was determined by quantifying the hydroxyproline content using the (dimethylamino)benzaldehyde and chloramine $\mathrm{T}$ assay and a hydroxyproline to collagen ratio of 1:7.69. As the gelatin within the GelMA group is derived from collagenous material, day 1 data is not included in relevant figures because the hydroxyproline assay detects the gelatin in the GelMA day 1 samples. Therefore, day 1 data is subtracted from the day 21 and day 42 data for all hydrogel groups to determine the actual produced collagen during in vitro culture.

\subsection{Histological and Immunohistochemical analysis}

Scaffolds were fixed with $4 \%$ paraformaldehyde solution, dehydrated in graded series of ethanol, embedded in paraffin wax, sectioned at $8 \mu \mathrm{m}$ and affixed to microscope slides. The sections were stained with Alcian Blue/Aldehyde Fuchsin, Picrosirius Red and Alizarin Red to assess for sGAG, collagen and calcium content.

Immunohistochemical technique was used to evaluate collagen types II and X. Sections were rehydrated and treated with chondroitinase ABC (Sigma-Aldrich) in a humidified environment at $37{ }^{\circ} \mathrm{C}$ to enhance permeability of the extracellular matrix. This was followed by incubation in goat serum to block non-specific sites and the relevant collagen type II (sc52658, 1:400) (mouse monoclonal; Santa Cruz Biotechnology) or collagen type X (ab49945, 1:200) primary antibodies (mouse monoclonal; Abcam) were applied overnight at $4{ }^{\circ} \mathrm{C}$. Treatment with peroxidase preceded the application of the secondary antibody (collagen type II, B7151, 1:300; collagen type X, ab49760, 1:200) at room temperature for $1 \mathrm{~h}$. Thereafter, all sections were incubated with ABC reagent (Vectastain PK-400; Vector Labs) for 45 min. Finally, sections were developed with DAB peroxidase (Vector Labs) for $5 \mathrm{~min}$. Positive and negative controls were included in the immunohistochemical staining protocols.

\subsection{Mechanical characterization}

All mechanical experiments were performed at room temperature $\left(\sim 25^{\circ} \mathrm{C}\right)$ using a twin column Zwick universal testing machine (Zwick, Roell). Unconfined compression tests were carried out in a PBS bath using a $100 \mathrm{~N}$ load cell. To ensure contact between the surface of the scaffolds $(n=4$ per group) and the top compression platen, a preload of $0.05 \mathrm{~N}$ was used for hydrogels alone constructs $(2 \mathrm{~mm}$ high x $6 \mathrm{~mm}$ diameter), whereas $0.5 \mathrm{~N}$ preload was applied to PCL alone as well as composite constructs (9 $\mathrm{mm} \times 9 \mathrm{~mm} \times 4 \mathrm{~mm}$ ). Hydrogels alone constructs were produced in the shape of a cylinder (A combined stress-relaxation and dynamic compression protocol (Supplementary Figure 1a) was implemented, where a series of compressive strains were applied in increasing steps of $10 \%$ to a maximum of $30 \%$. Peak strain was reached within $500 \mathrm{~s}$ and the equilibrium stress was obtained after a relaxation time of $45 \mathrm{~min}$. After the relaxation phase, five compressive cycles at $1 \%$ strain at a frequency of $1 \mathrm{~Hz}$ were superimposed. Ramp (or compressive), equilibrium and dynamic moduli were quantified at each strain increment. The ramp modulus was calculated as the slope of the initial linear region of the obtained stress-strain curves. The equilibrium modulus was determined as the equilibrium force divided by the sample's cross-sectional area divided by the applied strain. The dynamic modulus was measured as the average force amplitude over the five cycles divided by the sample's crosssectional area divided by the applied strain amplitude.

Cell-laden 3D bioprinted constructs underwent a shorter version of the stress-relaxation and dynamic compression protocol, to prevent cell death as there was no temperature and/or oxygen control during testing. Samples $(n=3)$ were subjected to $20 \%$ compressive strain followed by $45 \mathrm{~min}$ relaxation time and five dynamic compressive cycles at $1 \%$ strain and $1 \mathrm{~Hz}$ frequency. Ramp, equilibrium and dynamic moduli were quantified as explained in the previous paragraph.

Uniaxial tensile tests on 3D printed dog bone-shaped PCL samples were performed. Each sample ( $n=4$ per group) was mounted on a sandpaper frame with Araldite glue (Huntsman). The sandpaper frame, along with the PCL scaffold attached to it, was then fixed between two grips. Every test started after cutting through both edges of the sandpaper frame to allow for stretching of the sample. Tensile specimens were tested using a $2500 \mathrm{~N}$ load cell at a displacement rate of $1 \mathrm{~mm} / \mathrm{min}$ and loaded to $50 \%$ strain. The tensile modulus was taken as the slope of the stress-strain plots between 2 and $7 \%$ strain.

\subsection{Finite Element Analysis}

IPN only, PCL only and composite constructs $(4.5 \mathrm{~mm} \mathrm{x}$ $4.5 \mathrm{~mm} \times 4 \mathrm{~mm}$ ) were modelled using axisymmetric $\mathrm{FE}$ models developed in Abaqus 6.14 (DS Simulia, USA) to evaluate von Mises stress, maximum principal stress, hydrostatic pressure and scaffold lateral expansion upon loading application. PCL structures were modelled according to a previously described approach [65]. PCL reinforced IPN constructs were simulated by placing an IPN hydrogel matrix inside the pores of the PCL scaffold. A tie interaction between the PCL and IPN meshes was applied in order to constrain the translation degrees of freedom of the common nodes of the 
Table 2. Material parameters for the PCL and IPN hydrogel used for the numerical analysis.

IPN to those of the PCL scaffold. The models simulated ramp compression tests in unconfined configuration, until $10 \%$ strain was applied. For all groups, the nodes at the bottom ends of the scaffolds were constrained only in the loading direction, allowing for scaffold lateral expansion in the other two directions. A displacement of approximately $0.4 \mathrm{~mm}$ was prescribed through a platen that was considered a rigid body and in full contact with the nodes at the top surface of the scaffolds. Symmetric boundary conditions were applied to the constructs' inner faces.

\section{Material Material Model Material Properties}

\begin{tabular}{|c|c|c|}
\hline PCL & Isotropic elastic & $\mathrm{E}=430 \mathrm{MPa} ; v=0.3$ \\
& Isotropic plastic & $\begin{array}{c}\sigma_{\text {true }}^{\mathrm{y}}=17.745 \mathrm{MPa} ; \varepsilon_{\text {true }}^{\mathrm{y}}=0 ; \\
\sigma_{\text {true }}^{\mathrm{f}}=113.39 \mathrm{MPa} ; \varepsilon_{\text {true }}^{\mathrm{f}}=1.3316\end{array}$ \\
\hline IPN & Isotropic elastic & $\mathrm{E}=0.036 \mathrm{MPa} ; v=0.49$ \\
\hline
\end{tabular}

The engineering compressive modulus of the constructs was obtained from the stress and strain values of the linear region of the curve, calculated from the displacement and resultant reaction force data computed from the simulations. Quadratic ten-node tetrahedral elements (C3D10) were used. Material properties were determined from experimental mechanical testing. Isotropic elastic behaviour was assumed for the IPN hydrogel with a Poisson's ratio of 0.49, representing an almost incompressible material [66]. Based on our previous work [65], isotropic elastic and plastic material properties were assigned to the PCL. Material properties of both PCL and IPN are summarized in Table 2.

\subsection{Statistical analysis}

Results are presented as mean \pm standard deviation. Statistical analysis was performed with GraphPad (GraphPad Software, La Jolla California, USA). Experimental groups were analysed for significant differences using either a t-test or a general linear model for analysis of variance (ANOVA) and preforming Tukey's post-test. For all comparisons, significance was accepted for $\mathrm{p} \leq 0.05$.

\section{Results and discussion}

\subsection{Alginate - GeIMA IPNs possessed superior mechanical properties to their single hydrogel components}

The mechanical properties of alginate - GelMA IPN hydrogels were first compared to that of the individual hydrogel components. This was achieved using a series of stress-relaxation tests combined with dynamic tests in an unconfined compression configuration (Supplementary Figure 1a). The ramp phase of the unconfined compression test clearly showed that the IPN hydrogel was significantly stiffer than either the alginate or GelMA hydrogel alone (Supplementary Figure 1b). From the ramp phase of each compression test (to either $10 \%, 20 \%$ or $30 \%$ strain), it was possible to quantify the ramp modulus of the hydrogels (Figure 1a; Supplementary Figure 2 a-c; Table 3). The ramp modulus of IPN hydrogel was significantly higher than the alginate and GelMA only hydrogels, and significantly higher than the sum of the individual hydrogel components (shown by the red dashed line) at 20\% and 30\% strain, demonstrating a synergistic increase in stiffness. Strain stiffening was observed for all three hydrogels, with the ramp modulus increasing with the applied strain amplitude.

Equilibrium and dynamic properties are other important functional parameters to measure when assessing articular cartilage and biomaterials designed to regenerate this tissue [1, $3,5,67]$. Stress-relaxation tests were next used to determine the equilibrium modulus of the hydrogels in the absence of fluid flow. Stress-time curves (Supplementary Figure 1c) revealed the distinct stress-relaxation behaviour of each hydrogel. Alginate gels exhibited a complete relaxation of the stress after only $900 \mathrm{~s}$, presumably due to the unbinding of the ionic crosslinker followed by polymer matrix flow [68-71]. In contrast, GelMA hydrogels displayed relatively little stress 
cartilage is associated with the low permeability of the solid matrix $[3,4]$.

In spite of the improvements associated with the IPN hydrogel, its equilibrium and dynamic mechanical properties still remained at least one order of magnitude lower than that of native articular cartilage (equilibrium modulus ranges from approximately 0.2 to $2 \mathrm{MPa}$, while the dynamic modulus ranges from approximately 10 to $60 \mathrm{MPa})[15,72-74]$.

Table 3. Summary of constructs mechanical properties under unconfined compression.

\begin{tabular}{|c|c|c|c|c|c|c|c|c|c|}
\hline & \multicolumn{3}{|c|}{$\begin{array}{c}\text { Ramp Modulus } \\
\text { (MPa) }\end{array}$} & \multicolumn{3}{|c|}{$\begin{array}{l}\text { Equilibrium Modulus } \\
\text { (MPa) }\end{array}$} & \multicolumn{3}{|c|}{$\begin{array}{c}\text { Dynamic Modulus } \\
\text { (MPa) }\end{array}$} \\
\hline & $\begin{array}{l}10 \% \\
\text { strain }\end{array}$ & $\begin{array}{c}20 \% \\
\text { strain }\end{array}$ & $\begin{array}{l}30 \% \\
\text { strain }\end{array}$ & $\begin{array}{l}10 \% \\
\text { strain }\end{array}$ & $\begin{array}{l}20 \% \\
\text { strain }\end{array}$ & $\begin{array}{l}30 \% \\
\text { strain }\end{array}$ & $\begin{array}{l}10 \% \\
\text { strain }\end{array}$ & $\begin{array}{l}20 \% \\
\text { strain }\end{array}$ & $\begin{array}{c}30 \% \\
\text { strain }\end{array}$ \\
\hline $3.5 \%$ Alginate & $\begin{array}{c}0.011 \pm \\
0.003\end{array}$ & $\begin{array}{c}0.044 \pm \\
0.031\end{array}$ & $\begin{array}{c}0.049 \pm \\
0.026\end{array}$ & $\begin{array}{c}0.001 \pm \\
0.001\end{array}$ & $\begin{array}{c}0.006 \pm \\
0.005\end{array}$ & $\begin{array}{c}0.007 \pm \\
0.007\end{array}$ & $\begin{array}{c}0.046 \pm \\
0.034\end{array}$ & $\begin{array}{c}0.066 \pm \\
0.058\end{array}$ & $\begin{array}{c}0.094 \pm \\
0.095\end{array}$ \\
\hline $5 \%$ GelMA & $\begin{array}{c}0.008 \pm \\
0.001\end{array}$ & $\begin{array}{c}0.015 \pm \\
0.003\end{array}$ & $\begin{array}{c}0.018 \pm \\
0.002\end{array}$ & $\begin{array}{c}0.005 \pm \\
0.002\end{array}$ & $\begin{array}{c}0.005 \pm \\
0.003\end{array}$ & $\begin{array}{c}0.007 \pm \\
0.002\end{array}$ & $\begin{array}{c}0.015 \pm \\
0.006\end{array}$ & $\begin{array}{c}0.018 \pm \\
0.005\end{array}$ & $\begin{array}{c}0.027 \pm \\
0.005\end{array}$ \\
\hline$I P N$ & $\begin{array}{c}0.036 \pm \\
0.009\end{array}$ & $\begin{array}{c}0.110 \pm \\
0.035\end{array}$ & $\begin{array}{c}0.214 \pm \\
0.048\end{array}$ & $\begin{array}{c}0.013 \pm \\
0.011\end{array}$ & $\begin{array}{c}0.028 \pm \\
0.005\end{array}$ & $\begin{array}{c}0.034 \pm \\
0.001\end{array}$ & $\begin{array}{c}0.147 \pm \\
0.044\end{array}$ & $\begin{array}{c}0.265 \pm \\
0.070\end{array}$ & $\begin{array}{c}0.385 \pm \\
0.090\end{array}$ \\
\hline $\begin{array}{c}\text { Double Offset } \\
\text { PCL }\end{array}$ & $\begin{array}{c}0.221 \pm \\
0.060\end{array}$ & $\begin{array}{c}0.304 \pm \\
0.041\end{array}$ & $\begin{array}{c}0.623 \pm \\
0.092\end{array}$ & $\begin{array}{c}0.246 \pm \\
0.041\end{array}$ & $\begin{array}{c}0.261 \pm \\
0.043\end{array}$ & $\begin{array}{c}0.395 \pm \\
0.036\end{array}$ & $\begin{array}{c}0.515 \pm \\
0.127\end{array}$ & $\begin{array}{c}0.777 \pm \\
0.117\end{array}$ & $\begin{array}{c}1.603 \pm \\
0.205\end{array}$ \\
\hline $\begin{array}{c}\text { Double Offset } \\
P C L+I P N\end{array}$ & $\begin{array}{c}0.775 \pm \\
0.081\end{array}$ & $\begin{array}{c}1.034 \pm \\
0.378\end{array}$ & $\begin{array}{c}1.445 \pm \\
0.256\end{array}$ & $\begin{array}{c}0.203 \pm \\
0.215\end{array}$ & $\begin{array}{c}0.652 \pm \\
0.284\end{array}$ & $\begin{array}{c}0.957 \pm \\
0.527\end{array}$ & $\begin{array}{c}0.757 \pm \\
0.195\end{array}$ & $\begin{array}{c}1.415 \pm \\
0.285\end{array}$ & $\begin{array}{c}2.293 \pm \\
0.352\end{array}$ \\
\hline
\end{tabular}

a

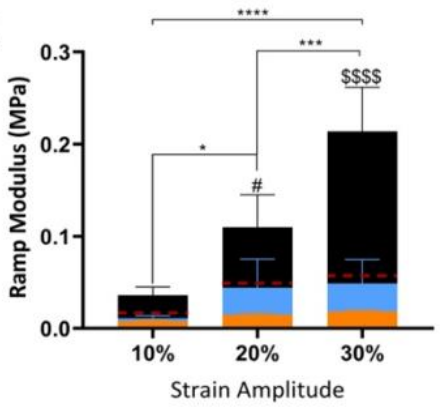

$3.5 \%$ Alginate b

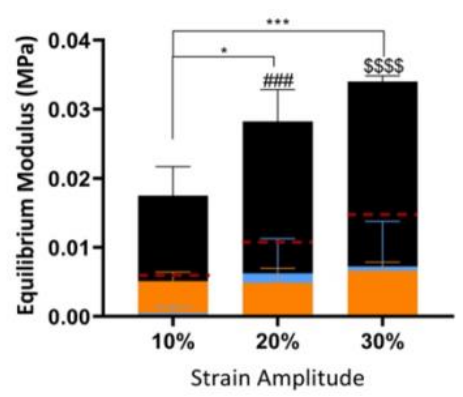

C

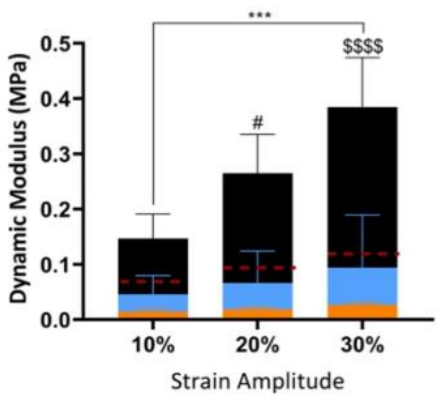

$5 \%$ GelMA

Figure 1. (a) Ramp modulus, (b) equilibrium modulus and (c) dynamic modulus in unconfined compression of $3.5 \%$ alginate (blue bars), 5\% GelMA (orange bars) and IPN (black bars) hydrogels when applying increasing levels of strain amplitude: 10, 
properties of articular cartilage, we next sought to investigate whether it was possible to design more biomimetic materials with nonlinear mechanical properties by reinforcing IPN hydrogels with 3D printed PCL networks with distinct tension-compression nonlinearity.
Recognizing that hydrogels alone [43, 75-79], even IPN

hydrogels, fail to recapitulate the complex mechanical

20 and $30 \%$. The sum of the modulus of the single component hydrogels for each applied strain is indicated by the dashed red line. \#p<0.01and \#\#\#p<0.001 IPN vs. 3.5\% alginate and $5 \%$ GelMA at $20 \%$ applied strain; $\$ \$ \$ p<0.0001$ IPN vs. $3.5 \%$ alginate and $5 \%$ GelMA at $30 \%$ strain amplitude; ${ }^{*} p<0.01, * * * p<0.001$ and $* * * * p<0.0001$ vs. IPN; $n=4$ per group.

\subsection{D printing of PCL networks with tension- compression nonlinearity}

Table 4. Summary of compressive and tensile properties for Aligned and Double Offset PCL.

\begin{tabular}{|c|c|c|}
\hline & $\begin{array}{c}\text { Compressive Modulus } \\
\mathbf{( M P a )}\end{array}$ & $\begin{array}{c}\text { Tensile Modulus } \\
\mathbf{( M P a )}\end{array}$ \\
\hline Aligned PCL & $1.341 \pm 0.095$ & $14.795 \pm 0.278$ \\
\hline Double Offset PCL & $0.218 \pm 0.014$ & $8.358 \pm 0.990$ \\
\hline
\end{tabular}

a Aligned
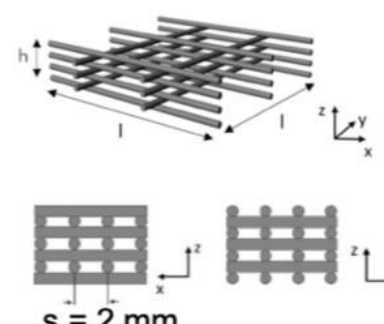
$\mathrm{s}=2 \mathrm{~mm}$
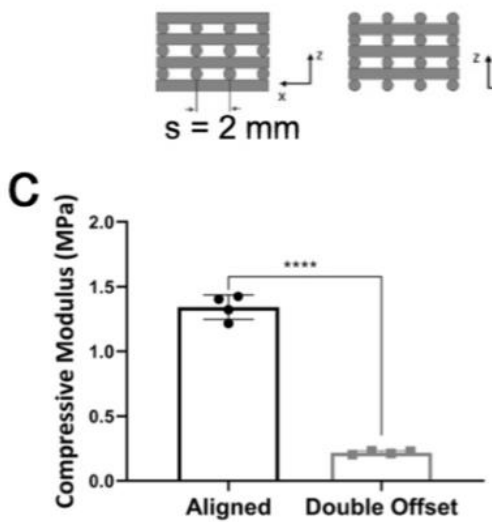

Double Offset

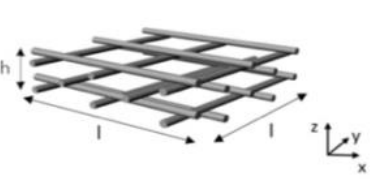

\section{${ }^{2} \mathrm{~L}$}

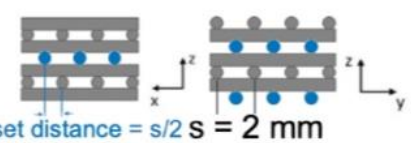

d

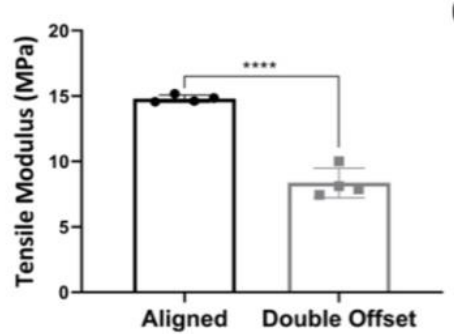

b Aligned Double Offset

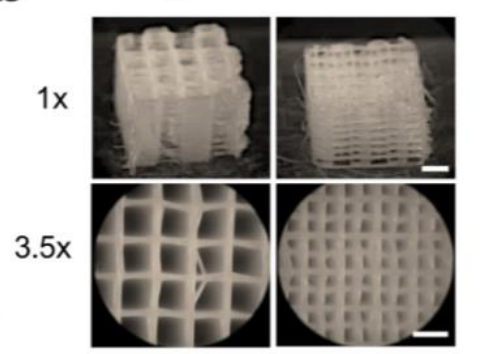

e

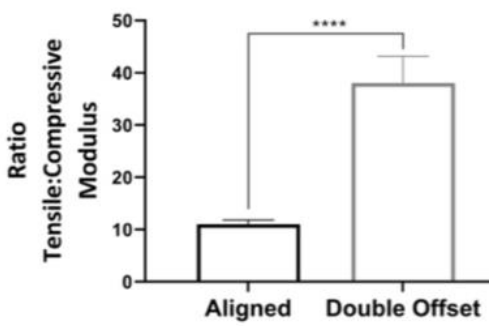

Figure 2. (a) Schematic of the PCL scaffolds depicting the Aligned geometry characterized by a regular orthogonal architecture and the Double Offset geometry comprising of offset layers present in both xz and yz planes. Offset layers are shown in blue. Scaffold architecture features are also indicated: $h$, height of the scaffold; l, length of the scaffold; s, fibre spacing. (b) Microscopy images of Aligned (left column) and Double Offset (right column) constructs. Scale bar: 2 mm. (c) Tensile modulus, (d) compressive modulus and (e) ratio between tensile and compressive modulus of Aligned (black bars) and Double Offset (grey bars) 3D printed PCL scaffolds. $* * * * p<0.0001 ; n=4$ per group. 
To this end, 3D printing parameters (Table 1) were varied in order to obtain two distinct 3D PCL geometries named Aligned and Double Offset (Figure 2a, b). The Aligned geometry (Figure 2a) was characterized by a regular orthogonal architecture in a lay-down pattern of $0^{\circ} 00^{\circ} / 90^{\circ} 90^{\circ}$ forming square pores. The Double Offset geometry (Figure 2a) consisted of an orthogonal architecture with the same laydown pattern. This differed from the Aligned pattern because of the presence of offset layers every other four layers that were deposited at an offset distance equal to half the fibre spacing. The obtained 3D network/scaffold architectures were tested in uniaxial tension and compression configurations to evaluate the effect of network geometry on the degree of tension-compression nonlinearity. Aligned constructs were stiffer in both tension and compression compared to Double Offset networks (Figure 2c, d; Table 4), with more dramatic differences observed in the compressive properties of the PCL networks. The compressive modulus was $1.341 \pm 0.095$ and $0.218 \pm 0.014 \mathrm{MPa}$ for Aligned and Double Offset samples respectively (Figure 2c; Table 4), whereas he tensile stiffness was $14.795 \pm 0.278 \mathrm{MPa}$ for the Aligned geometry and 8.358 $\pm 0.990 \mathrm{MPa}$ for the Double Offset one (Figure 2d; Table 4). When quantifying the ratio between tensile and compressive moduli, higher tension-compression nonlinearity was observed in the Double Offset networks in comparison to Aligned samples (Figure 2e).

\subsection{Combining IPN hydrogels with reinforcing $P C L$ networks to produce biomimetic composites with mechanical properties comparable to native cartilage}

As tension-compression nonlinearity is believed to play a critical role in the load-bearing capacity of articular cartilage by enhancing fluid pressurization under compression, we hypothesized that reinforcing IPN hydrogels with a Double Offset PCL network design would result in the development of constructs that more closely mimicked the mechanical behaviour of the native tissue. To test this hypothesis, we next reinforced the alginate - GelMA IPNs with 3D printed PCL networks with two distinct architectures (Aligned and Double Offset).

a

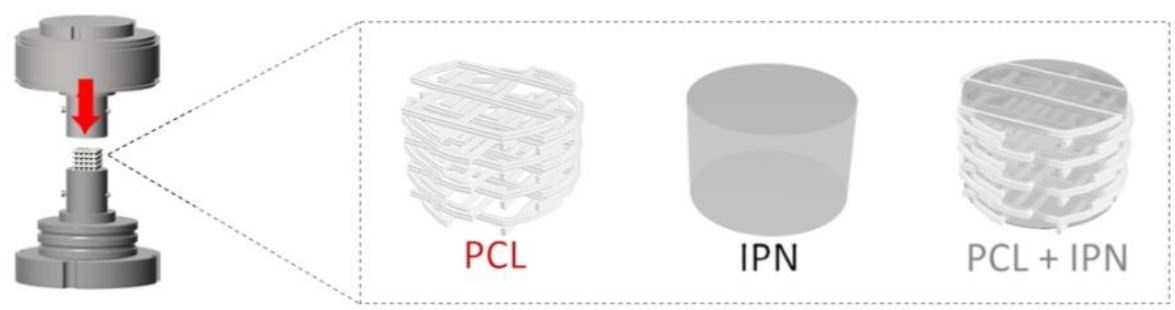

b

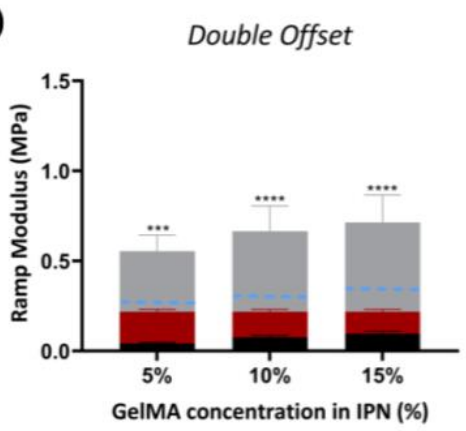

C

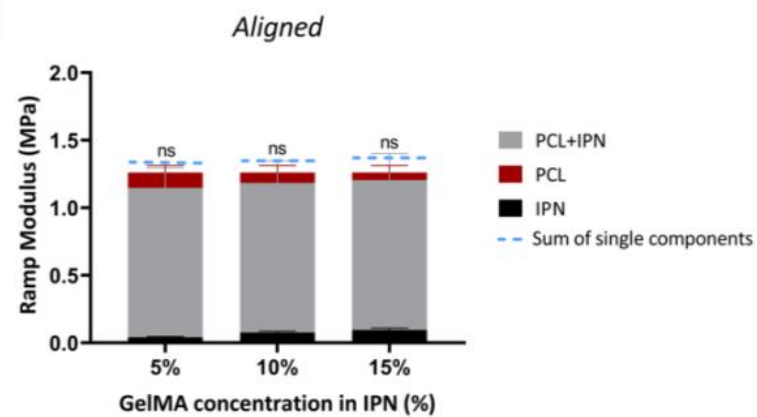

Figure 3. (a) Schematic illustration of the mechanical testing set-up and scaffold groups (PCL, IPN and $P C L+I P N)$ that underwent compression tests. (b,c) Ramp modulus of PCL frames (red bars), IPN hydrogels (black bars) and PCL + IPN scaffolds (grey bars) when varying GeIMA concentration in the IPN from 5 to $15 \%$ (w/v). Results are shown for groups containing either the (b) Aligned or (c) Double Offset PCL geometry. The sum of the compressive modulus of the individual components for each GelMA concentration is indicated by the dashed blue line. ${ }^{* * *} p<0.001$ and ${ }^{* * * *} p<0.0001 ; n=4$ per group. 
a

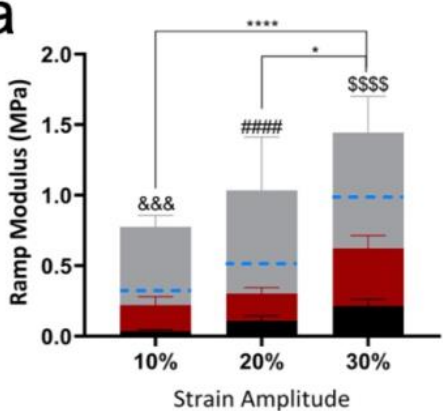

b

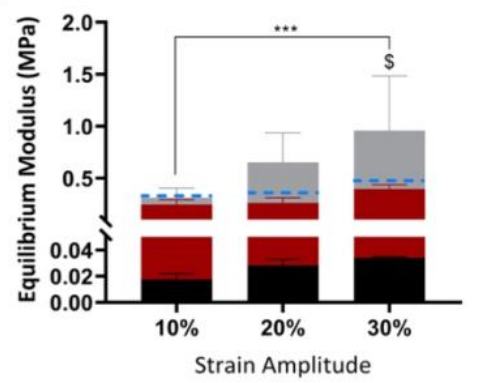

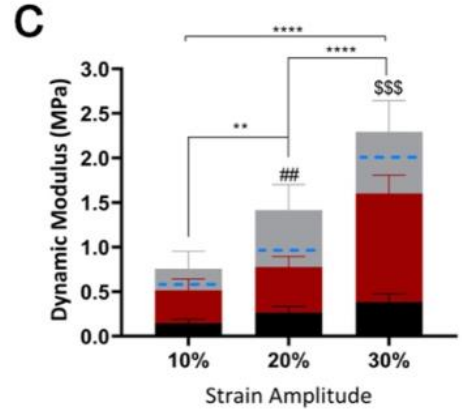

\section{IPN $\square \mathrm{PCL} \square \mathrm{PCL}+\mathrm{IPN}--$ Sum of single components}

Figure 4. (a) Ramp modulus, (b) equilibrium modulus and (c) dynamic modulus in unconfined compression of IPN hydrogel (black bars), Double Offset PCL (red bars) and PCL + IPN (grey bars) scaffolds when applying increasing levels of strain amplitude: 10, 20 and 30\%. The sum of the moduli of the individual components for each applied strain is indicated by the dashed blue line. \&\&\&p<0.001 PCL + IPN vs. PCL at $10 \%$ applied strain; $\# p<0.01$ and \#\#\# $p<0.0001 P C L+I P N$ vs. PCL at $20 \%$ applied strain; $\$ p<0.05$, $\$ \$ p<0.001$ and $\$ \$ \$ p<0.0001 P C L+I P N$ vs. $P C L$ at $30 \%$ applied strain; $* p<0.05, * * p<0.01, * * * p<0.001$ and $* * * * p<0.0001$ vs. $P C L+I P N ; n=4$ per group.

The PCL scaffolds were infused with different alginate GelMA IPN hydrogel formulations where the alginate concentration $(3.5 \% \mathrm{w} / \mathrm{v})$ was maintained constant, whereas the GelMA content was increased $(5,10$ and $15 \% \mathrm{w} / \mathrm{v})$. The resulting composite constructs were subjected to compression tests to determine their ramp modulus in comparison to PCL scaffolds alone, IPNs alone and the sum of the moduli of the individual components (Figure 3a-c; Supplementary Figure 3). After infusing the Double Offset PCL scaffolds with the IPN hydrogels (Figure 3b), a synergistic increase in stiffness was observed for all IPN formulations. Specifically, the ramp modulus of the composite samples (grey bars) was higher than the sum of the moduli of the single components (blue dashed lines) as well as to the individual components, i.e. the PCL scaffolds (red bars) and IPN gels (black bars). The ramp modulus of the composite increased as the GelMA content in the IPN increased $(0.554 \pm 0.090,0.665 \pm 0.140$ and $0.713 \pm$ $0.153 \mathrm{MPa}$ at 5,10 and $15 \% \mathrm{w} / \mathrm{v}$ GelMA concentration, respectively). Moreover, Double Offset PCL + IPN constructs were significantly stiffer than the Double Offset PCL alone samples $(0.218 \pm 0.014 \mathrm{MPa})$, showing approximately $2.5-, 3$ and 3.3-fold increases in stiffness at 5, 10 and $15 \% \mathrm{w} / \mathrm{v}$ GelMA concentrations, respectively. The obtained ramp modulus values for the Double Offset PCL + IPN constructs fell in the range of native tissue properties ranging from approximately 0.24 to $1.4 \mathrm{MPa}[73,80,81]$. Combining the Aligned PCL constructs with the IPN hydrogels (Figure 3c) did not result in the same synergistic increase in stiffness. The ramp moduli of the composite constructs did not increase as the GelMA content in the IPN gels increased $(1.147 \pm 0.149$, $1.183 \pm 0.164$ and $1.206 \pm 0.195 \mathrm{MPa}$ at 5,10 and $15 \% \mathrm{w} / \mathrm{v}$
GelMA concentration, respectively). Furthermore, there was no statistical difference in ramp modulus compared to the Aligned PCL alone scaffolds (1.261 $\pm 0.051 \mathrm{MPa})$, indicating that the PCL component was dominating the mechanical behaviour of the composite.

The ramp, equilibrium and dynamic moduli of the composites were also assessed after the application of 10, 20 and 30\% strain for the Double Offset PCL design (Figure 4ac; Supplementary Figure 4; Table 3). Stiffness was found to increase with increases in the applied strain amplitude. For all strain levels, the ramp moduli was significantly higher for the composite constructs (grey bars; $0.775 \pm 0.081,1.034 \pm 0.378$ and $1.445 \pm 0.256 \mathrm{MPa}$ when applying 10, 20 and $30 \%$ strains respectively) compared to the PCL alone (red bars; $0.221 \pm$ $0.060,0.304 \pm 0.041$ and $0.623 \pm 0.092 \mathrm{MPa}$ at 10,20 and $30 \%$ applied strain amplitudes respectively), the IPN alone (black bars) as well as the sum of the individual components (blue dashed line) at all applied strain amplitudes (Figure 4a; Supplementary Figure 4 a-c; Table 3). The equilibrium modulus of the PCL + IPN composites was only higher than the PCL scaffolds alone at higher strain levels.

In unconfined compression, the equilibrium modulus of articular cartilage ranges from approximately 0.2 to $2 \mathrm{MPa}$ $[67,73,74,82-84]$, whereas the dynamic modulus in response to cyclic loading ranges from approximately 10 to $60 \mathrm{MPa}$, depending on the loading frequency $[15,31,73,85,86]$. The composite 3D printed PCL reinforced IPN constructs possessed equilibrium moduli that fell in the range of native cartilage equilibrium properties (Figure 4b; Supplementary Figure 4 d-f; Table 3). Although the dynamic modulus of PCL + IPN composites did not match the values of the native tissue, 
a

Double Offset PCL

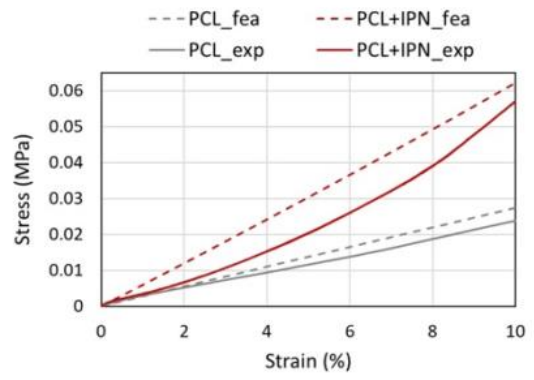

C

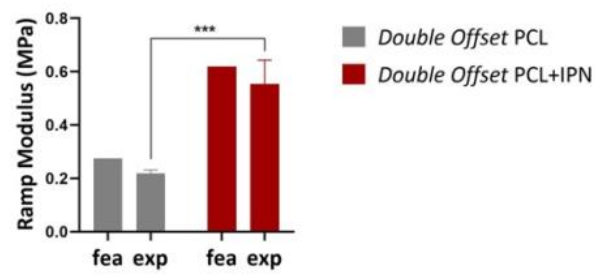

e

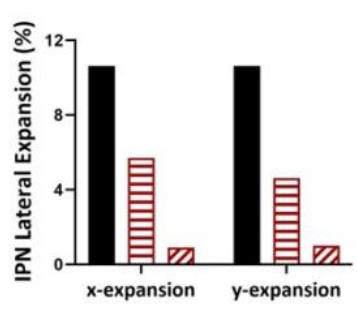

it showed a marked improvement compared to PCL and IPN alone (Figure 4c; Supplementary Figure 4 g-i; Table 3), reaching the same order of magnitude as articular cartilage. The dynamic modulus for composite constructs was measured to be $0.757 \pm 0.195,1.415 \pm 0.285$ and $2.293 \pm 0.352 \mathrm{MPa}$ at 10,20 and $30 \%$ applied strain amplitudes respectively, fact its permeability is significantly higher than that of articular cartilage. While a lower permeability (and hence a superior capacity to generate fluid load support) could be achieved by increasing the density of the IPN, this could negatively impact nutrient transport and waste removal once the biomaterial is seeded with cells for tissue engineering applications. Hence, the properties achieved here can be viewed as a balance between providing initial mechanical function (mimicking native equilibrium and ramp modulus values) and an environment supportive of chondrogenesis (that is assessed below).

b Aligned PCL

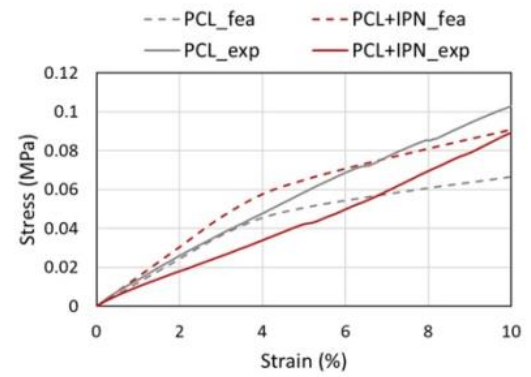

d

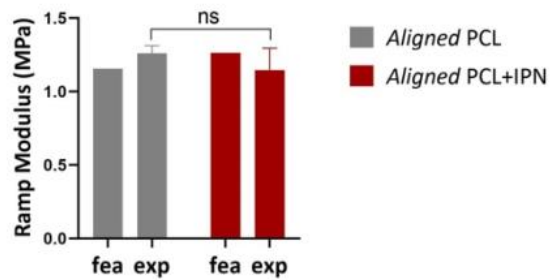

$f$

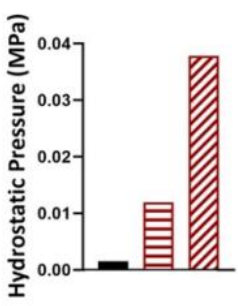

IPN alone

曰 Aligned PCL+IPN

$\square$ Double Offset PCL+IPN

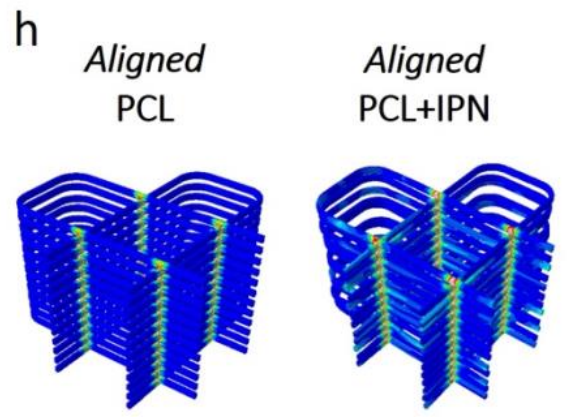

Figure 5. Computational and experimental $(\mathrm{a}, \mathrm{b})$ compressive stress-strain curves and $(\mathrm{c}, \mathrm{d})$ ramp modulus for $(\mathrm{a}, \mathrm{c})$ Double Offset and (b,d) Aligned PCL-based structures; ${ }^{* * *} \mathrm{p}<0.001 ; \mathrm{n}=4$ per group. Comparison of predicted IPN (e) lateral expansion 
and (f) hydrostatic pressure in IPN alone, Aligned PCL+IPN and Double Offset PCL+IPN structures. Von Mises stress contour plots for (g) Double Offset and (h) Aligned PCL-based constructs.

\subsection{FE modelling to better understand the mechanism by which 3D printed fibre networks mechanically reinforce IPN hydrogels}

FEA was next utilized to better understand the mechanism by which the PCL networks were mechanically reinforcing the IPN hydrogels.

FE simulations were performed of uniaxial compression tests on the IPN alone, PCL alone and composite constructs for both Double Offset and Aligned PCL-based structures (Figure 5 ). The predicted stress-strain behaviour (Figure 5a, b) and compressive modulus (Figure $5 \mathrm{c}, \mathrm{d}$ ) of all groups were in good agreement with the experimental results. Having demonstrated that this computational approach was able to accurately predict the compressive mechanical behaviour of 3D printed scaffolds, we next sought to investigate how the Double Offset PCL network was reinforcing the IPN hydrogels. When articular cartilage is subjected to compressive loading, the collagen network resists lateral expansion and fluid pressurization supports a significant component of the applied load. When comparing the expansion in both $\mathrm{x}$ - and y-direction of IPN in Double Offset PCL + IPN, Aligned PCL + IPN and IPN alone models, it was observed that reinforcing the IPN with either of the PCL networks reduced lateral expansion of the composite, with the lowest expansion observed in the Double Offset PCL composite (Figure 5e). We next predicted the average hydrostatic pressure produced in the IPN when applying $10 \%$ compressive strain. In general, higher pressure was generated within the IPN in the presence of both Aligned and Double Offset PCL, with the highest values predicted in Double Offset PCL + IPN structures. Compared to IPN alone, there was approximately a 24- and 7.5-fold increase in hydrostatic pressure in Double Offset PCL + IPN and Aligned PCL + IPN constructs, respectively (Figure 5f). The FE model also predicted higher stresses in the PCL filaments within the Double Offset PCL network in composites compared to Double Offset PCL only structures (Figure 5g) On the other side, little difference in von Mises stress distribution was predicted in the PCL network of the Aligned PCL + IPN composites compared to Aligned PCL only scaffolds (Figure $5 \mathrm{~h})$. Together, the FE models suggest two related mechanisms by which the Double Offset PCL network reinforces the IPN hydrogel. Firstly, the presence of the IPN in the PCL network increases the tensile stresses generated in the PCL filaments (see also Supplementary Figure 5), allowing them to play a greater role in supporting the applied load. Secondly, the PCL network is predicted to limit lateral expansion of the composite and lead to higher magnitudes of hydrostatic pressure being generated within the IPN. Such reinforcement mechanisms are enhanced by PCL networks with greater tension-compression nonlinearity.

Other studies have reported similar reinforcement mechanisms, mainly when reinforcing hydrogels with electrospun fibres. For example, Castilho et al. [66] demonstrated that the reinforcement effect of microfibre reinforced GelMA composites derived either from fibres being pulled in tension by the expansion of the hydrogel, or from the support provided by the hydrogel in preventing buckling of the fibres under compression.

The FE simulations further demonstrate that the combination of the Double Offset PCL network with the IPN produce a cartilage biomimetic construct.

\subsection{PCL reinforced cell-laden IPNs fabricated using 3D bioprinting support chondrogenesis with minimal hypertrophy}

Having successfully obtained composite constructs with mechanical properties comparable to those of native cartilage, we proceeded to assess the capacity of these composites to provide a pro-chondrogenic environment in vitro. With a view to bioprinting cell-laden implants, we established a multipletool biofabrication process that first involved the bioprinting of the porous reinforcing Double Offset PCL networks in the shape of a cylinder $(6 \mathrm{~mm} \times 6 \mathrm{~mm})$. In a second bioprinting step, the obtained PCL networks were placed in a moulding system that kept them in the centre of the well of a 6 -well plate, which facilitated the z-direction bioprinting of the bioinks (alginate, GelMA and alginate - GelMA IPNs containing a co-culture of BMSCs and CCs) into the PCL networks (Figure 6a). Z-direction bioprinting consists of depositing the cell encapsulating-bioinks within the empty pores of the previously printed PCL networks by moving the needle in the z-direction. The bioprinted constructs were then transferred to chondrogenic media and cultured in static conditions at $5 \% \mathrm{O}_{2}$ for the first 3 weeks and then at $21 \% \mathrm{O}_{2}$ for further 3 weeks.

To verify that cell viability was not affected by the 3D printing process or the post-fabrication crosslinking of the bioinks, all constructs were analysed using live-dead staining after $24 \mathrm{~h}$ of culture (Figure 6b-c). All bioinks supported high levels of cell viability ( $80 \%$; Figure $6 \mathrm{c})$, showing that the fabrication process was not overly harmful to the cells.

Over 6 weeks of in vitro culture, the ramp modulus of the PCL reinforced GelMA and IPN-based composites progressively increased (Figure 6d-f; Table 5). 
a

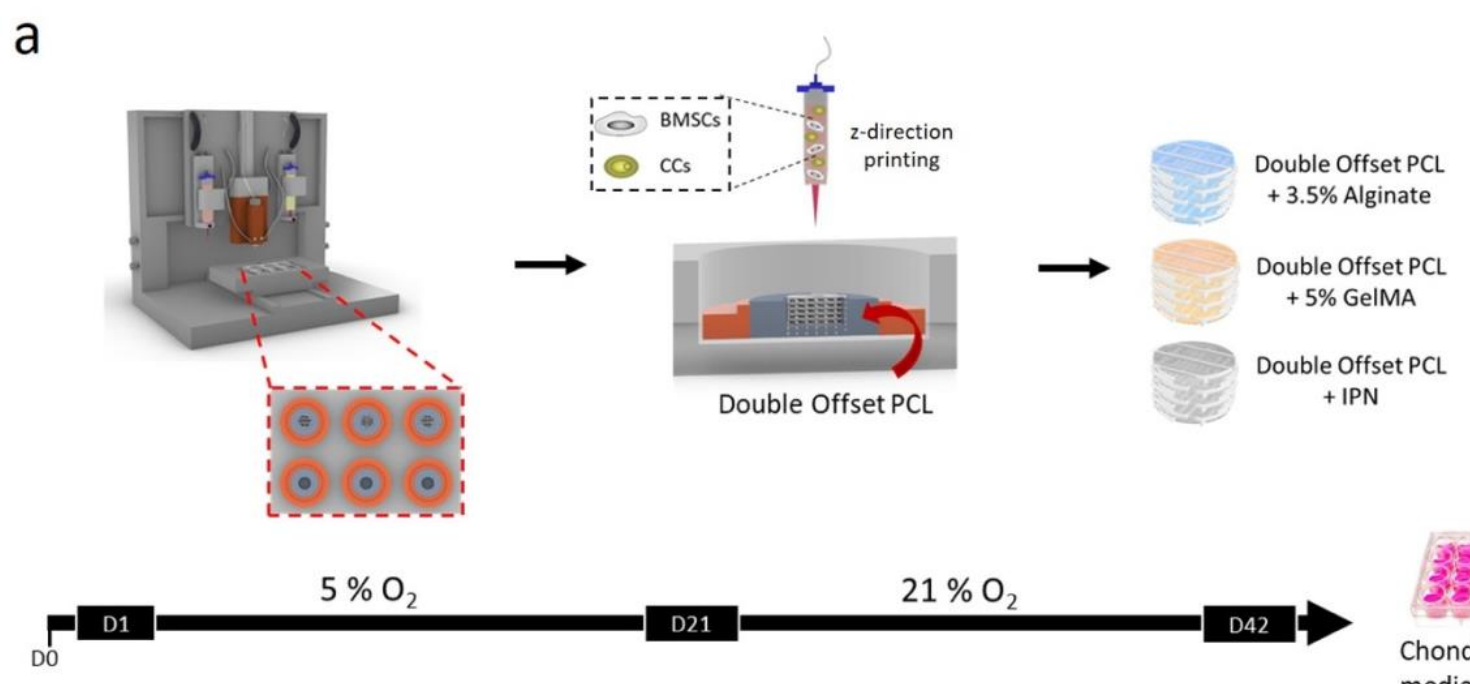

b

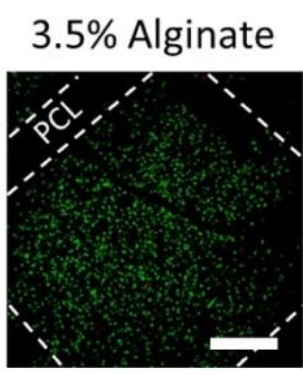

\section{$5 \%$ GelMA}
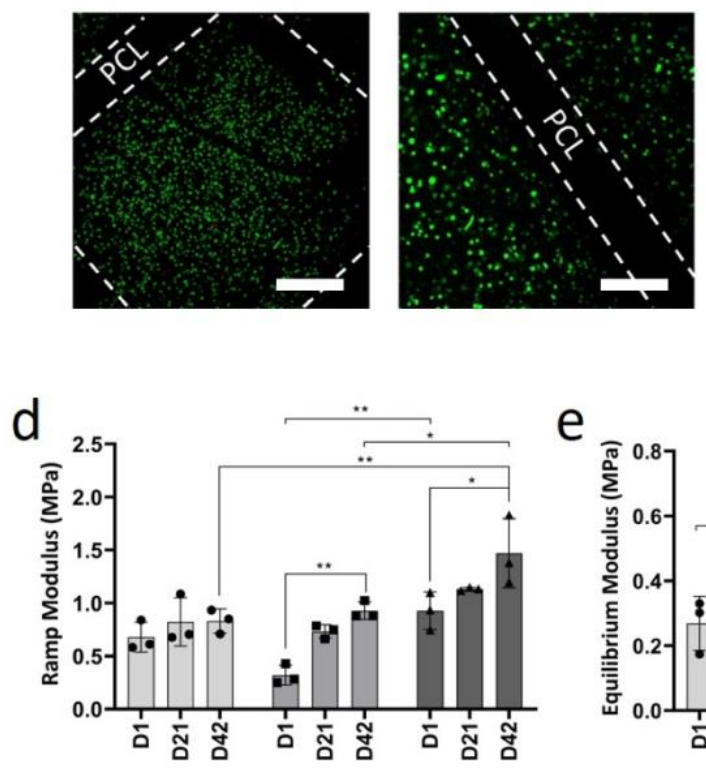

e

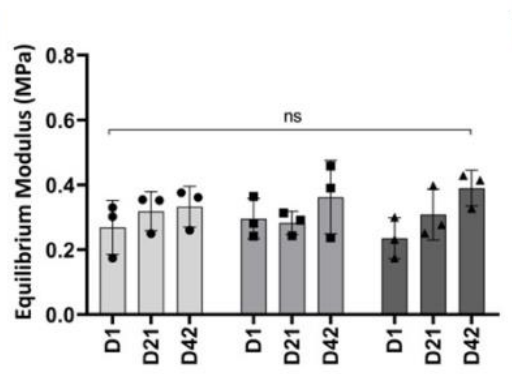

3.5\% Alginate

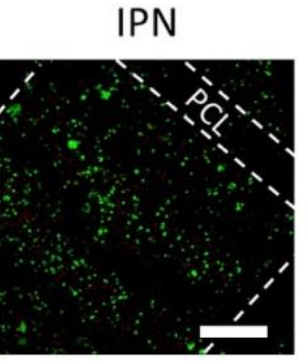

C

C Day 1 media (+TGF- $\beta 3$ )
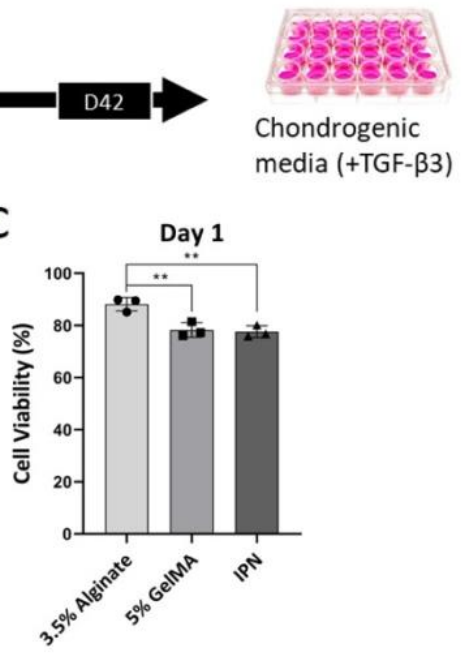

$f$

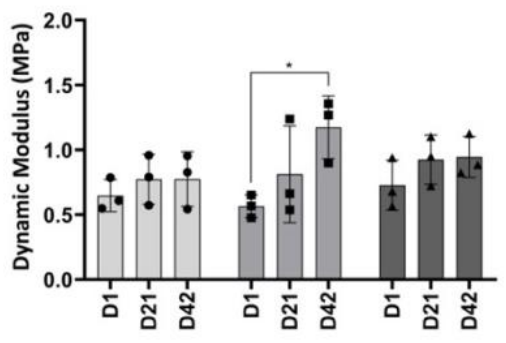

IPN

Figure 6. (a) Schematic of the experimental set-up: Double Offset PCL frames were 3D printed via FDM and then placed in a moulding system that kept the PCL scaffolds in the centre of each well of a 6-well plate to facilitate microextrusion (z-direction printing) of the cell-laden bioinks. Obtained composite constructs were cultured in chondrogenic media for the first 3 weeks in hypoxia and the following 3 weeks in normoxia. (b) Representative images of Live/Dead staining used to determine the cell viability of 3.5\% alginate, 5\% GeIMA and IPN bioinks. Scale bar: $200 \mu \mathrm{m}$. (c) Quantitative analysis of the cell viability for all of the bioinks. (d) Ramp modulus, (e) equilibrium modulus and ( $f$ ) dynamic modulus for all composite groups after day 1, 21 and 42 of culture. ${ }^{*} p<0.05,{ }^{* *} p<0.01, * * * p<0.001$ and ${ }^{* * * *} p<0.0001 ; n=3$ per group. 
Table 5. Summary of mechanical properties of alginate-, GelMA- and IPN-based composites under unconfined compression after 1,21 and 42 days of culture.

\begin{tabular}{|c|c|c|c|c|c|c|c|c|c|}
\hline \multirow{2}{*}{} & \multicolumn{3}{|c|}{ Ramp Modulus } & \multicolumn{3}{c|}{ Equilibrium Modulus } & \multicolumn{3}{c|}{ Dynamic Modulus } \\
& \multicolumn{3}{|c|}{ (MPa) } & \multicolumn{3}{c|}{ (MPa) } & \multicolumn{3}{c|}{ (MPa) } \\
\cline { 2 - 12 } & $\mathrm{D} 1$ & $\mathrm{D} 21$ & $\mathrm{D} 42$ & $\mathrm{D} 1$ & $\mathrm{D} 21$ & $\mathrm{D} 42$ & $\mathrm{D} 1$ & $\mathrm{D} 21$ & $\mathrm{D} 42$ \\
\hline \multirow{2}{*}{$3.5 \%$ Alginate } & $0.678 \pm$ & $0.822 \pm$ & $0.830 \pm$ & $0.269 \pm$ & $0.318 \pm$ & $0.332 \pm$ & $0.647 \pm$ & $0.773 \pm$ & $0.774 \pm$ \\
& 0.141 & 0.228 & 0.114 & 0.083 & 0.060 & 0.063 & 0.123 & 0.193 & 0.211 \\
\hline $5 \%$ GelMA & $0.320 \pm$ & $0.732 \pm$ & $0.927 \pm$ & $0.296 \pm$ & $0.282 \pm$ & $0.362 \pm$ & $0.565 \pm$ & $0.812 \pm$ & $1.174 \pm$ \\
& 0.094 & 0.062 & 0.082 & 0.062 & 0.036 & 0.113 & 0.088 & 0.278 & 0.345 \\
\hline IPN & $0.926 \pm$ & $1.133 \pm$ & $1.469 \pm$ & $0.235 \pm$ & $0.309 \pm$ & $0.390 \pm$ & $0.727 \pm$ & $0.925 \pm$ & $0.945 \pm$ \\
& 0.177 & 0.015 & 0.326 & 0.063 & 0.078 & 0.055 & 0.192 & 0.190 & 0.158 \\
\hline
\end{tabular}

The PCL reinforced-IPN constructs possessed the highest ramp modulus at all time points reaching values of $1.469 \pm$ $0.326 \mathrm{MPa}$ compared to $0.830 \pm 0.114$ and $0.927 \pm 0.082 \mathrm{MPa}$ for alginate-based and GelMA-based composites respectively at day 42 (Figure 6d; Table 5). No major differences in the equilibrium modulus were observed between the different groups, which was approximately $0.350 \mathrm{MPa}$ after 42 days of culture (Figure 6e; Table 5). Significant increases in dynamic modulus with time in culture were only observed in the GelMA-based composites (Figure 6f; Table 5).

All three PCL reinforced bioinks supported the development of a hyaline cartilage-like tissue that stained positive for GAG and collagen type II deposition (Figure 7ac). GAG and collagen deposition appeared quite pericellular within the IPN-based composites at day 21 (Figure 7b) but become more diffuse with further time in culture (Figure 7c). What was presumed to be non-specific type $\mathrm{X}$ collagen staining, was observed in the GelMA-based composites at day 1 , making it difficult to assess type $\mathrm{X}$ collagen deposition in this group. There was no positive staining for collagen type $\mathrm{X}$ in the other groups at any time point, suggesting that the engineered tissues were not becoming hypertrophic with time in culture. Alginate and GelMA-based scaffolds showed some positive staining for alizarin red at day 42 , demonstrating the presence of calcium deposits. In contrast, no evidence of calcium deposition was observed in IPN-based composites, suggesting then the development of phenotypically stable articular cartilage-like tissue within the IPNs. The percentage of tissue staining positive for Alizarin Red was also quantified, which demonstrated that the alginate-based constructs supported the highest levels of calcification, while calcific deposits were barely detectable in the IPN group (Figure $6 \mathrm{~g}$ ). Histological observations were confirmed by biochemical analysis, which demonstrated that GAG and collagen content significantly increased over the culture period, with no dramatic differences observed between the three hydrogel groups (Figure 7e-f).

Together these results demonstrated that alginate - GelMA IPNs were able to support robust chondrogenesis, with levels of ECM deposition comparable to that observed in the individual hydrogels. Importantly, it was shown that the increased mechanical properties achieved with the IPN did not negatively affect its biological functionality. Usually, hydrogels presenting cell binding motifs such as GelMA can facilitate cell-cell and cell-matrix interactions leading more to fibrocartilage-like tissue formation [78, 87-90]. In contrast, alginate is known to be an inert material and should facilitate MSCs to develop a round shape, which is known to support a chondrogenic phenotype $[78,91,92]$. Given that a co-culture of CCs and MSCs is known to support the generation of phenotypically stable articular cartilage [64, 93-96], we did not expect to see dramatic difference in hypertrophy and progression along an endochondral pathway in the three different hydrogel bioink groups. Importantly, the stiffer IPN based composites supported the development of cartilage resistant to calcification after 6 weeks of culture, despite the fact that stiffer hydrogel environments can be more supportive of chondrocyte hypertrophy [37, 97-99].

Although the architecture of the 3D printed PCL networks did not mimic the arrangement of the collagen fibres in the native articular cartilage, the findings of this study show that the internal fibre pattern of such scaffolds can be tailored to ultimately engineer constructs with key mechanical properties (i.e. nonlinearity, equilibrium and dynamic properties) that match or approach those of cartilage prior to any in vitro culture, making them potentially suitable for immediate implantation. In addition, designing PCL networks with an offset pattern results in relatively high tension-compression 
nonlinearity, which, similarly to the collagen network in the native tissue, limits lateral expansion of the constructs and enhances the hydrostatic pressure within the IPN during compressive loading. Furthermore, the PCL + IPN composites support robust chondrogenesis, ensuring that the mechanical properties of cell-laden constructs will continue to improve (e.g. with time in culture) as the encapsulated cells secrete a cartilage-like ECM.

As the architecture of the collagenous network is known to playing a key role in determining the load-bearing properties of soft tissues such as articular cartilage [82], future studies could use 3D bioprinting strategies to develop scaffolds that also replicate specific structural characteristics of cartilage. Specifically, 3D bioprinting could potentially enable the production of either polymer networks that mimic the zonespecific arrangement of the collagen fibres in the native tissue, and/or polymeric fibre networks that could direct the alignment of the newly formed matrix to produce engineered tissues that better mimic the zonal collagenous structure of articular cartilage.

\section{a}

Alcian Blue-

Aldehyde Fuchsin

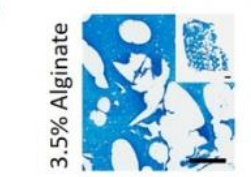

게웡

$\underset{a}{a}$

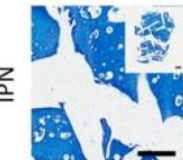

$\mathrm{b}$

C
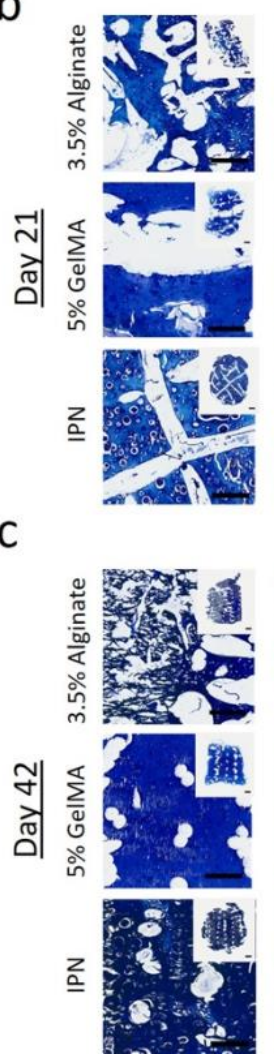

Alizarin Red

Picrosirius Red

Collagen II
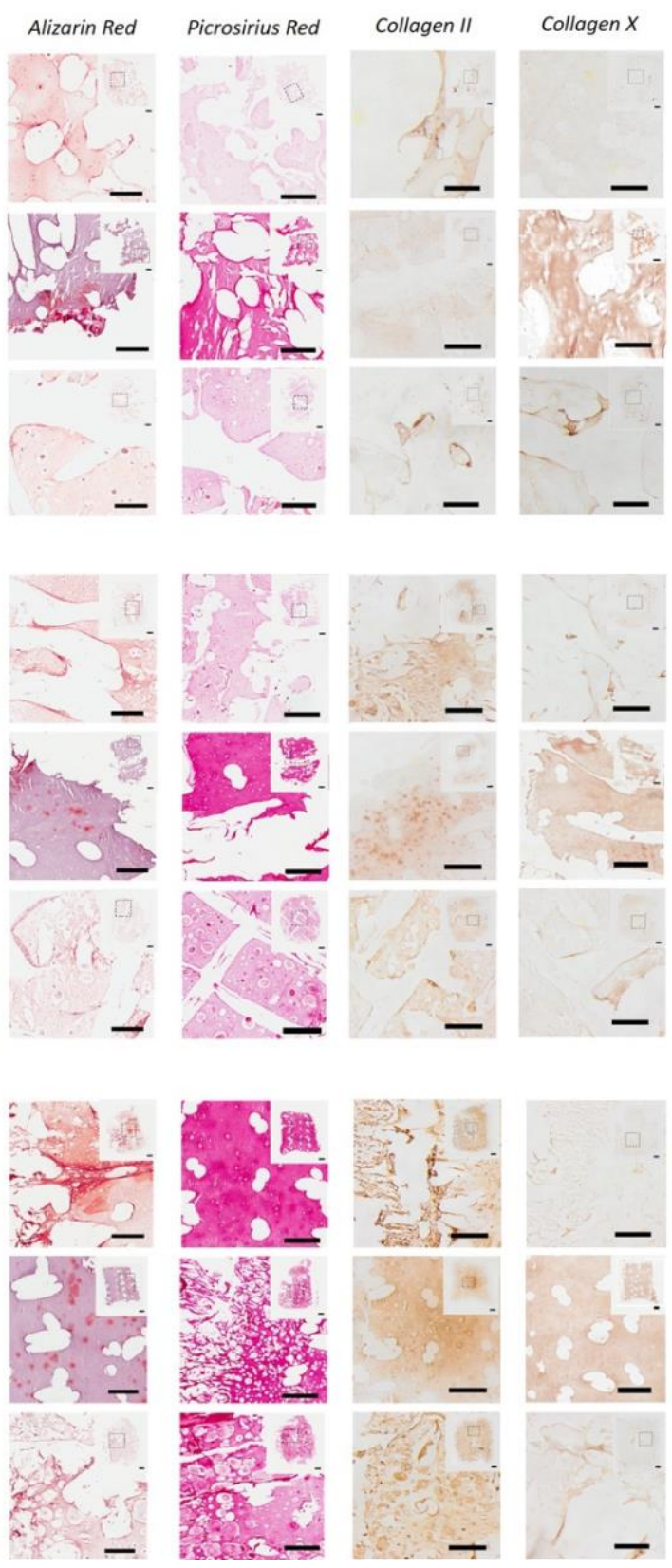

$3.5 \%$ Alginate

5\% GelMA

$\triangle$ IPN

d

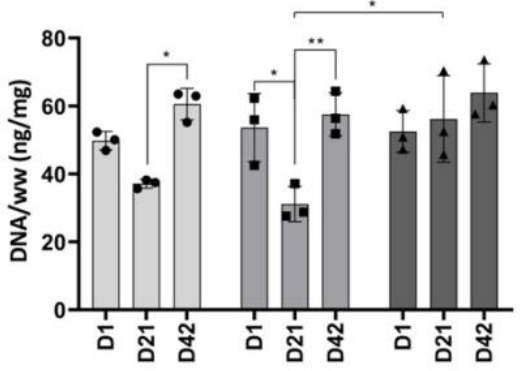

e

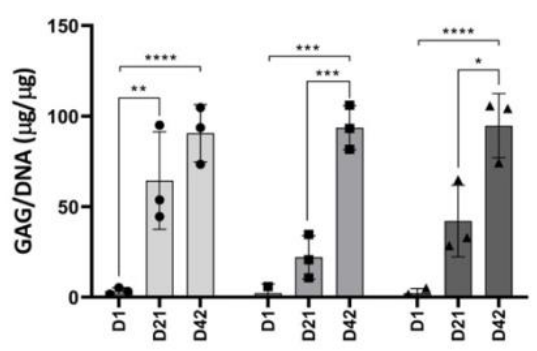

f

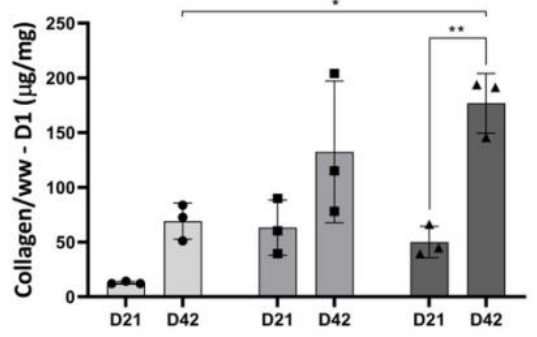

g

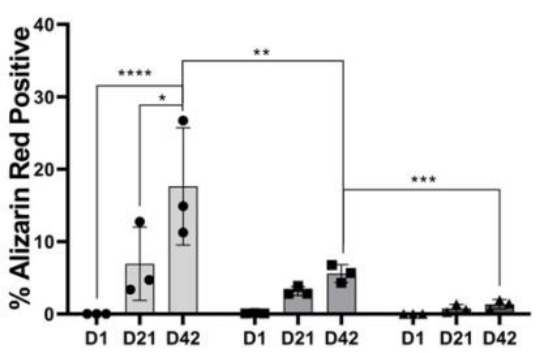


Figure 7. (a-c) Histological and immunohistochemical staining of all 3D bioprinted constructs groups after (a) 1 , (b) 21 and (c) 42 days of in vitro culture. Scale bar: $500 \mu \mathrm{m}$. (d-f) Biochemical analysis of all composites, including (d) DNA content normalized to wet weight (ng/mg), (e) GAG/DNA and (f) collagen/DNA after day 1, 21 and 42 of in vitro culture. (g) Quantification of percentage of Alizarin Red staining within all three composite groups at day 1,21 and $42 .{ }^{*} p<0.05, * * p<0.01$, $* * * \mathrm{p}<0.001$ and $* * * * \mathrm{p}<0.0001 ; \mathrm{n}=3$ per group.

\section{Conclusions}

This work represents a significant step towards developing biomechanically functional biomaterials for cartilage tissue engineering. The combination of alginate - GelMA IPN hydrogels with appropriately designed 3D printed PCL networks enables the engineering of composites with mechanical properties that are mimetic of normal articular cartilage. Importantly, such composite constructs provide encapsulated cells with an environment conducive to chondrogenesis, resulting in robust production of articular cartilage-like matrix.

\section{Acknowledgements}

This work was supported by a European Research Council Consolidator grant (647004) and a research grant from Science Foundation Ireland under grant number 12/IA/1554.

\section{References}

[1] Mow, V. C., S. C. Kuei, W. M. Lai and C. G. Armstrong 1980. Biphasic creep and stress relaxation of articular cartilage in compression? Theory and experiments. J Biomech Eng 102 73-84.

[2] Moutos, F. T., L. E. Freed and F. Guilak 2007. A biomimetic three-dimensional woven composite scaffold for functional tissue engineering of cartilage Nat Mater 6 162167.

[3] Gannon, A. R., T. Nagel and D. J. Kelly 2012. The role of the superficial region in determining the dynamic properties of articular cartilage Osteoarthritis Cartilage 20 1417-1425. [4] Soltz, M. A. and G. A. Ateshian 2000. Interstitial Fluid Pressurization during Confined Compression Cyclical Loading of Articular Cartilage Ann Biomed Eng 28 150-159. [5] Guilak, F., D. L. Butler and S. A. Goldstein 2001. Functional tissue engineering: the role of biomechanics in articular cartilage repair. Clin Orthop Relat Res S295-305.

[6] Horkay, F. 2012. Interactions of cartilage extracellular matrix macromolecules Journal of Polymer Science, Part B: Polymer Physics 50 1699-1705.

[7] Kempson, G. E., H. Muir, S. A. V. Swanson and M. A. R. Freeman 1970. Correlations between stiffness and the chemical constituents of cartilage on the human femoral head BBA - General Subjects 215 70-77.

[8] Buckwalter, J. A., P. J. Roughley and L. C. Rosenberg 1994. Age-Related changes in cartilage proteoglycans: Quantitative electron microscopic studies Microsc Res Tech 28 398-408.
[9] Pastrama, M.-I., A. C. Ortiz, L. Zevenbergen, N. Famaey, W. Gsell, C. P. Neu, U. Himmelreich and I. Jonkers 2019. Combined enzymatic degradation of proteoglycans and collagen significantly alters intratissue strains in articular cartilage during cyclic compression J Mech Behav Biomed Mater 98 383-394.

[10] Schmidt, M. B., V. C. Mow, L. E. Chun and D. R. Eyre 1990. Effects of proteoglycan extraction on the tensile behavior of articular cartilage J Orthop Res 8 353-363.

[11] Lu, X. L. and V. C. Mow 2008. Biomechanics of articular cartilage and determination of material properties Med Sci Sports Exerc 40 193-199.

[12] Bae, W. C., V. W. Wong, J. Hwang, J. M. Antonacci, G. E. Nugent-Derfus, M. E. Blewis, M. M. Temple-Wong and R. L. Sah 2008. Wear-lines and split-lines of human patellar cartilage: relation to tensile biomechanical properties. Osteoarthritis Cartilage 16 841-845.

[13] Kempson, G. E., M. A. Freeman and S. A. Swanson 1968. Tensile properties of articular cartilage. Nature $2201127-$ 1128.

[14] Williamson, A. K., A. C. Chen, K. Masuda, E. J. M. A. Thonar and R. L. Sah 2003. Tensile mechanical properties of bovine articular cartilage: Variations with growth and relationships to collagen network components J Orthop Res 21 872-880.

[15] Park, S., C. T. Hung and G. A. Ateshian 2004. Mechanical response of bovine articular cartilage under dynamic unconfined compression loading at physiological stress levels Osteoarthritis Cartilage 12 65-73.

[16] Park, S., R. Krishnan, S. B. Nicoll and G. A. Ateshian 2003. Cartilage interstitial fluid load support in unconfined compression J Biomech 36 1785-1796.

[17] Federico, S. and W. Herzog 2008. On the anisotropy and inhomogeneity of permeability in articular cartilage Biomech Model Mechanobiol 7 367-378.

[18] Canal Guterl, C., C. T. Hung and G. A. Ateshian 2010. Electrostatic and non-electrostatic contributions of proteoglycans to the compressive equilibrium modulus of bovine articular cartilage J Biomech 43 1343-1350.

[19] Bas, O., E. M. De-Juan-Pardo, C. Meinert, D. D'Angella, J. G. Baldwin, L. J. Bray, R. M. Wellard, S. Kollmannsberger, E. Rank, C. Werner, T. J. Klein, I. Catelas and D. W. Hutmacher 2017. Biofabricated soft network composites for cartilage tissue engineering Biofabrication 9025014.

[20] Almeida, H. V., G. M. Cunniffe, T. Vinardell, C. T. Buckley, F. J. O'Brien and D. J. Kelly 2015. Coupling Freshly Isolated CD44(+) Infrapatellar Fat Pad-Derived Stromal Cells with a TGF-beta3 Eluting Cartilage ECM-Derived Scaffold as 
a Single-Stage Strategy for Promoting Chondrogenesis $A d v$ Healthc Mater 4 1043-1053.

[21] Thorpe, S. D., T. Nagel, S. F. Carroll and D. J. Kelly 2013. Modulating gradients in regulatory signals within mesenchymal stem cell seeded hydrogels: a novel strategy to engineer zonal articular cartilage PLoS One 8 e60764.

[22] Cunniffe, G. M., P. J. Díaz-Payno, E. J. Sheehy, S. E. Critchley, H. V. Almeida, P. Pitacco, S. F. Carroll, O. R. Mahon, A. Dunne, T. J. Levingstone, C. J. Moran, R. T. Brady, F. J. O'Brien, P. A. J. Brama and D. J. Kelly 2019. Tissue-specific extracellular matrix scaffolds for the regeneration of spatially complex musculoskeletal tissues Biomaterials 188 63-73.

[23] Yang, J., Y. S. Zhang, K. Yue and A. Khademhosseini 2017. Cell-laden hydrogels for osteochondral and cartilage tissue engineering Acta Biomater 57 1-25.

[24] Bhattacharjee, M., J. Coburn, M. Centola, S. Murab, A. Barbero, D. L. Kaplan, I. Martin and S. Ghosh 2015. Tissue engineering strategies to study cartilage development, degeneration and regeneration Adv Drug Del Rev 84 107-122. [25] Kwon, H., W. E. Brown, C. A. Lee, D. Wang, N. Paschos, J. C. Hu and K. A. Athanasiou 2019. Surgical and tissue engineering strategies for articular cartilage and meniscus repair Nature Reviews Rheumatology.

[26] Izadifar, Z., X. Chen and W. Kulyk 2012. Strategic design and fabrication of engineered scaffolds for articular cartilage repair. Journal of functional biomaterials 3 799-838.

[27] Johnstone, B., M. Alini, M. Cucchiarini, G. Dodge, D. Eglin, F. Guilak, H. Madry, A. Mata, R. Mauck, C. Semino and M. Stoddart 2013. Tissue engineering for articular cartilage repair - the state of the art Eur Cells Mater 25 248267.

[28] Yang, X., Z. Lu, H. Wu, W. Li, L. Zheng and J. Zhao 2018. Collagen-alginate as bioink for three-dimensional (3D) cell printing based cartilage tissue engineering Materials Science and Engineering: C 83 195-201.

[29] Filardo, G., E. Kon, A. Roffi, A. Di Martino and M. Marcacci 2013. Scaffold-based repair for cartilage healing: A systematic review and technical note Arthroscopy - Journal of Arthroscopic and Related Surgery 29 174-186.

[30] Neumann, A. J., T. Quinn and S. J. Bryant 2016. Nondestructive evaluation of a new hydrolytically degradable and photo-clickable PEG hydrogel for cartilage tissue engineering Acta Biomater 39 1-11.

[31] Liao, I. C., F. T. Moutos, B. T. Estes, X. Zhao and F. Guilak 2013. Composite three-dimensional woven scaffolds with interpenetrating network hydrogels to create functional synthetic articular cartilage Adv Funct Mater 23 5833-5839. [32] Xu, C., G. Dai and Y. Hong 2019. Recent advances in high-strength and elastic hydrogels for 3D printing in biomedical applications Acta Biomater 95 50-59.

[33] Jeon, O., J.-Y. Shin, R. Marks, M. Hopkins, T.-H. Kim, H.-H. Park and E. Alsberg 2017. Highly Elastic and Tough Interpenetrating Polymer Network-Structured Hybrid Hydrogels for Cyclic Mechanical Loading-Enhanced Tissue Engineering Chem Mater 29 8425-8432.

[34] Brigham, M. D., M. Eng, A. Bick, E. Lo, A. Bendali, J. A. Burdick, D. Ph and A. Khademhosseini 2009.
Mechanically Robust and Bioadhesive Collagen SemiInterpenetrating Networks Tissue Engineering: Part A 15 1645-1653.

[35] Chimene, D., K. K. Lennox, R. R. Kaunas and A. K. Gaharwar 2016. Advanced Bioinks for 3D Printing: A Materials Science Perspective Ann Biomed Eng 44 20902102.

[36] Malda, J., J. Visser, F. P. Melchels, T. Jüngst, W. E. Hennink, W. J. A. Dhert, J. Groll and D. W. Hutmacher 2013. 25th anniversary article: Engineering hydrogels for biofabrication Adv Mater 25 5011-5028.

[37] Bian, L., C. Hou, E. Tous, R. Rai, R. L. Mauck and J. A. Burdick 2013. The influence of hyaluronic acid hydrogel crosslinking density and macromolecular diffusivity on human MSC chondrogenesis and hypertrophy Biomaterials 34 413-421.

[38] Sun, A. X., H. Lin, M. R. Fritch, H. Shen, P. G. Alexander, M. DeHart and R. S. Tuan 2017. Chondrogenesis of human bone marrow mesenchymal stem cells in 3dimensional, photocrosslinked hydrogel constructs: Effect of cell seeding density and material stiffness. Acta Biomater $\mathbf{5 8}$ 302-311.

[39] Visser, J., F. P. W. Melchels, J. E. Jeon, E. M. van Bussel, L. S. Kimpton, H. M. Byrne, W. J. a. Dhert, P. D. Dalton, D. W. Hutmacher and J. Malda 2015. Reinforcement of hydrogels using three-dimensionally printed microfibres. Nature communications 66933.

[40] Liao, I.-C., F. T. Moutos, B. T. Estes, X. Zhao and F. Guilak 2013. Composite Three-Dimensional Woven Scaffolds with Interpenetrating Network Hydrogels to Create Functional Synthetic Articular Cartilage Adv Funct Mater 23 5833-5839.

[41] Moffat, K. L., K. Goon, F. T. Moutos, B. T. Estes, S. J. Oswald, X. Zhao and F. Guilak 2018. Composite Cellularized Structures Created from an Interpenetrating Polymer Network Hydrogel Reinforced by a 3D Woven Scaffold Macromol Biosci 18001401800140.

[42] Murphy, S. V. and A. Atala 2014. 3D bioprinting of tissues and organs Nat Biotechnol 32 773-785.

[43] Daly, A. C., F. E. Freeman, T. Gonzalez-Fernandez, S. E. Critchley, J. Nulty and D. J. Kelly 2017. 3D Bioprinting for Cartilage and Osteochondral Tissue Engineering Adv Healthc Mater 6.

[44] Middleton, J. C. and A. J. Tipton 2000. Synthetic biodegradable polymers as orthopedic devices Biomaterials 21 2335-2346.

[45] Woodruff, M. A. and D. W. Hutmacher 2010. The return of a forgotten polymer - Polycaprolactone in the 21 st century Progress in Polymer Science (Oxford) 35 1217-1256.

[46] Olubamiji, A. D., Z. Izadifar, J. L. Si, D. M. L. Cooper, B. F. Eames and D. X. B. Chen 2016. Modulating mechanical behaviour of 3D-printed cartilage-mimetic PCL scaffolds: influence of molecular weight and pore geometry. Biofabrication 8025020.

[47] Shasteen, C. and Y. B. Choy 2011. Controlling degradation rate of poly(lactic acid) for its biomedical applications Biomedical Engineering Letters 1 163-167. 
[48] Pan, Z. and J. Ding 2012. Poly(lactide-co-glycolide) porous scaffolds for tissue engineering and regenerative medicine Interface Focus 2 366-377.

[49] Nair, L. S. and C. T. Laurencin 2007. Biodegradable polymers as biomaterials Progress in Polymer Science (Oxford) 32 762-798.

[50] Dawson, E., G. Mapili, K. Erickson, S. Taqvi and K. Roy 2008. Biomaterials for stem cell differentiation Adv Drug Del $\operatorname{Rev} 60$ 215-228.

[51] van Sliedregt, A., M. Knook, S. C. Hesseling, H. K. Koerten, K. de Groot and C. A. van Blitterswijk 1992. Cellular reaction on the intraperitoneal injection of four types of polylactide particulates Biomaterials 13 819-824.

[52] Hung, C. T., R. L. Mauck, C. C. B. Wang, E. G. Lima and G. A. Ateshian 2004. A paradigm for functional tissue engineering of articular cartilage via applied physiologic deformational loading Ann Biomed Eng 32 35-49.

[53] Fedorovich, N. E., J. Alblas, J. R. de Wijn, W. E. Hennink, A. J. Verbout and W. J. A. Dhert 2007. Hydrogels as Extracellular Matrices for Skeletal Tissue Engineering: State-of-the-Art and Novel Application in Organ Printing Tissue Eng 13 1905-1925.

[54] Augst, A. D., H. J. Kong and D. J. Mooney 2006. Alginate hydrogels as biomaterials Macromol Biosci 6623 633.

[55] Gasperini, L., J. F. Mano and R. L. Reis 2014. Natural polymers for the microencapsulation of cells Journal of the Royal Society Interface 11.

[56] Alsberg, E., H. J. Kong, Y. Hirano, M. K. Smith, A. Albeiruti and D. J. Mooney 2003. Regulating bone formation via controlled scaffold degradation J Dent Res 82 903-908. [57] Lee, K. Y. and D. J. Mooney 2012. Alginate: Properties and biomedical applications Progress in Polymer Science (Oxford) 37 106-126.

[58] Jeon, O., C. Powell, S. M. Ahmed and E. Alsberg 2010. Biodegradable, photocrosslinked alginate hydrogels with independently tailorable physical properties and cell adhesivity Tissue Engineering - Part A 16 2915-2925.

[59] Jeon, O., J. Y. Shin, R. Marks, M. Hopkins, T. H. Kim, H. H. Park and E. Alsberg 2017. Highly elastic and tough interpenetrating polymer network-structured hybrid hydrogels for cyclic mechanical loading-enhanced tissue engineering Chem Mater 29 8425-8432.

[60] Sun, J. Y., X. Zhao, W. R. K. Illeperuma, O. Chaudhuri, K. H. Oh, D. J. Mooney, J. J. Vlassak and Z. Suo 2012. Highly stretchable and tough hydrogels Nature 489 133-136.

[61] Zhu, M., Y. Wang, G. Ferracci, J. Zheng, N. J. Cho and B. H. Lee 2019. Gelatin methacryloyl and its hydrogels with an exceptional degree of controllability and batch-to-batch consistency Sci Rep 9 1-13.

[62] Wang, H., L. Zhou, J. Liao, Y. Tan, K. Ouyang, C. Ning, G. Ni and G. Tan 2014. Cell-laden photocrosslinked GelMADexMA copolymer hydrogels with tunable mechanical properties for tissue engineering J Mater Sci Mater Med 25 2173-2183.

[63] Van Den Bulcke, A. I., B. Bogdanov, N. De Rooze, E. H. Schacht, M. Cornelissen and H. Berghmans 2000. Structural and Rheological Properties of Methacrylamide Modified Gelatin Hydrogels Biomacromolecules 1 31-38.

[64] Mesallati, T., E. J. Sheehy, T. Vinardell, C. T. Buckley and D. J. Kelly 2015. Tissue engineering scaled-up, anatomically shaped osteochondral constructs for joint resurfacing Eur Cells Mater 30 163-186.

[65] Schipani, R., D. R. Nolan, C. Lally, D. J. Kelly, R. Schipani, D. R. Nolan, C. Lally and D. J. Kelly 2019. Integrating finite element modelling and $3 \mathrm{D}$ printing to engineer biomimetic polymeric scaffolds for tissue engineering 8207.

[66] Castilho, M., G. Hochleitner, W. Wilson, B. van Rietbergen, P. D. Dalton, J. Groll, J. Malda and K. Ito 2018. Mechanical behavior of a soft hydrogel reinforced with threedimensional printed microfibre scaffolds Sci Rep 81245.

[67] Mow, V. C. and X. E. Guo 2002. MechanoElectrochemical Properties Of Articular Cartilage: Their Inhomogeneities and Anisotropies Annu Rev Biomed Eng 4 175-209.

[68] Gu, L., O. Chaudhuri, H.-p. Lee, D. J. Mooney and M. E. Levenston 2017. Mechanical confinement regulates cartilage matrix formation by chondrocytes Nature Materials 161243 1251.

[69] Chaudhuri, O., L. Gu, D. Klumpers, M. Darnell, S. A. Bencherif, J. C. Weaver, N. Huebsch, H. P. Lee, E. Lippens, G. N. Duda and D. J. Mooney 2016. Hydrogels with tunable stress relaxation regulate stem cell fate and activity Nat Mater 15 326-334.

[70] Metzger, T. A., S. A. Schwaner, A. J. LaNeve, T. C. Kreipke and G. L. Niebur 2015. Pressure and shear stress in trabecular bone marrow during whole bone loading $J$ Biomech 48 3035-3043.

[71] Zhao, X., N. Huebsch, D. J. Mooney and Z. Suo 2010. Stress-relaxation behavior in gels with ionic and covalent crosslinks J Appl Phys 10763509.

[72] Beck, E. C., M. Barragan, M. H. Tadros, S. H. Gehrke and M. S. Detamore 2016. Approaching the compressive modulus of articular cartilage with a decellularized cartilagebased hydrogel Acta Biomater 38 94-105.

[73] Little, C. J., N. K. Bawolin and X. Chen 2011. Mechanical Properties of Natural Cartilage and TissueEngineered Constructs Tissue Engineering Part B: Reviews 17 213-227.

[74] Jurvelin, J. S., M. D. Buschmann and E. B. Hunziker 1997. Optical and mechanical determination of Poisson's ratio of adult bovine humeral articular cartilage J Biomech 30235 241.

[75] Erickson, I. E., A. H. Huang, C. Chung, R. T. Li, J. A. Burdick and R. L. Mauck 2009. Differential Maturation and Structure-Function Relationships in Mesenchymal Stem Celland Chondrocyte-Seeded Hydrogels Tissue Engineering Part A 15 1041-1052.

[76] Hofmann, S., S. Knecht, R. Langer, D. L. Kaplan, G. Vunjak-Novakovic, H. P. Merkle and L. Meinel 2006. Cartilage-like Tissue Engineering Using Silk Scaffolds and Mesenchymal Stem Cells Tissue Eng 0060915113954001. 
[77] Critchley, S. E. and D. J. Kelly 2017. Bioinks for bioprinting functional meniscus and articular cartilage Journal of 3D Printing in Medicine 1 269-290.

[78] Daly, A. C., S. E. Critchley, E. M. Rencsok and D. J. Kelly 2016. A comparison of different bioinks for 3D bioprinting of fi brocartilage and hyaline cartilage Biofabrication 8 1-10.

[79] Critchley, S., G. Cunniffe, A. O'Reilly, P. Diaz-Payno, R. Schipani, A. McAlinden, D. Withers, J. Shin, E. Alsberg and D. J. Kelly 2019. Regeneration of Osteochondral Defects Using Developmentally Inspired Cartilaginous Templates Tissue Eng Part A 25 159-171.

[80] Beck, E. C., M. Barragan, M. H. Tadros, S. H. Gehrke and M. S. Detamore 2016. Approaching the compressive modulus of articular cartilage with a decellularized cartilagebased hydrogel. Acta Biomater 38 94-105.

[81] Korhonen, R. K. and J. S. Jurvelin 2010. Compressive and tensile properties of articular cartilage in axial loading are modulated differently by osmotic environment Med Eng Phys 32 155-160

[82] Gannon, A. R., T. Nagel, A. P. Bell, N. C. Avery and D. J. Kelly 2015. Postnatal changes to the mechanical properties of articular cartilage are driven by the evolution of its Collagen network European Cells and Materials 29 105-123.

[83] Gao, L. L., C. Q. Zhang, H. Gao, Z. D. Liu and P. P. Xiao 2014. Depth and rate dependent mechanical behaviors for articular cartilage: Experiments and theoretical predictions Mater Sci Eng, C 38 244-251.

[84] Athanasiou, K. A., A. Agarwal and F. J. Dzida 1994. Comparative study of the intrinsic mechanical properties of the human acetabular and femoral head cartilage J Orthop Res 12 340-349.

[85] Kinnunen, J., S. Saarakkala, M. Hauta-Kasari, P. Vahimaa and J. S. Jurvelin 2011. Optical spectral reflectance of human articular cartilage - relationships with tissue structure, composition and mechanical properties Biomedical Optics Express 21394.

[86] Bian, L., E. G. Lima, S. L. Angione, K. W. Ng, D. Y. Williams, D. Xu, A. M. Stoker, J. L. Cook, G. A. Ateshian and C. T. Hung 2008. Mechanical and biochemical characterization of cartilage explants in serum-free culture. $J$ Biomech 41 1153-1159.

[87] Levett, P. A., F. P. W. Melchels, K. Schrobback, D. W. Hutmacher, J. Malda and T. J. Klein 2014. A biomimetic extracellular matrix for cartilage tissue engineering centered on photocurable gelatin, hyaluronic acid and chondroitin sulfate Acta Biomater 10 214-223.

[88] Levett, P. A., F. P. W. Melchels, K. Schrobback, D. W. Hutmacher, J. Malda and T. J. Klein 2014. Chondrocyte redifferentiation and construct mechanical property development in single-component photocrosslinkable hydrogels Journal of Biomedical Materials Research - Part A 102 2544-2553.

[89] Schuurman, W., P. a. Levett, M. W. Pot, P. R. van Weeren, W. J. a. Dhert, D. W. Hutmacher, F. P. W. Melchels, T. J. Klein and J. Malda 2013. Gelatin-methacrylamide hydrogels as potential biomaterials for fabrication of tissueengineered cartilage constructs. Macromol Biosci 13 551-561.
[90] Awad, H. A., M. Q. Wickham, H. A. Leddy, J. M. Gimble and F. Guilak 2004. Chondrogenic differentiation of adiposederived adult stem cells in agarose, alginate, and gelatin scaffolds Biomaterials 25 3211-3222.

[91] Rowley, J. A., G. Madlambayan and D. J. Mooney 1999. Alginate hydrogels as synthetic extracellular matrix materials Biomaterials 20 45-53.

[92] Benya, P. D. and J. D. Shaffer 1982. Dedifferentiated chondrocytes reexpress the differentiated collagen phenotype when cultured in agarose gels Cell 30 215-224.

[93] Fischer, J., A. Dickhut, M. Rickert and W. Richter 2010. Human articular chondrocytes secrete parathyroid hormonerelated protein and inhibit hypertrophy of mesenchymal stem cells in coculture during chondrogenesis Arthritis Rheum 62 2696-2706.

[94] Bian, L., D. Y. Zhai, R. L. Mauck and J. A. Burdick 2011. Coculture of human mesenchymal stem cells and articular chondrocytes reduces hypertrophy and enhances functional properties of engineered cartilage Tissue Engineering - Part A 17 1137-1145.

[95] Kang, N., X. Liu, Y. Guan, J. Wang, F. Gong, X. Yang, L. Yan, Q. Wang, X. Fu, Y. Cao and R. Xiao 2012. Effects of co-culturing BMSCs and auricular chondrocytes on the elastic modulus and hypertrophy of tissue engineered cartilage Biomaterials 33 4535-4544.

[96] Cooke, M. E., A. A. Allon, T. Cheng, A. C. Kuo, H. T. Kim, T. P. Vail, R. S. Marcucio, R. A. Schneider, J. C. Lotz and T. Alliston 2011. Structured three-dimensional co-culture of mesenchymal stem cells with chondrocytes promotes chondrogenic differentiation without hypertrophy. Osteoarthritis Cartilage 19 1210-1218.

[97] Engler, A. J., S. Sen, H. L. Sweeney and D. E. Discher 2006. Matrix Elasticity Directs Stem Cell Lineage Specification Cell 126 677-689.

[98] Nguyen, Q. T., Y. Hwang, A. C. Chen, S. Varghese and R. L. Sah 2012. Cartilage-like mechanical properties of poly (ethylene glycol)-diacrylate hydrogels Biomaterials 33 66826690.

[99] Zhu, D., X. Tong, P. Trinh and F. Yang 2018. Mimicking Cartilage Tissue Zonal Organization by Engineering TissueScale Gradient Hydrogels as 3D Cell Niche Tissue Engineering - Part A 24 1-10. 